\title{
Olfactory Bulb Deep Short-Axon Cells Mediate Widespread Inhibition of Tufted Cell Apical Dendrites
}

\author{
(DShawn D. Burton, ${ }^{1,2}$ Greg LaRocca, ${ }^{3}{ }^{\circledR}$ Annie Liu, ${ }^{2,4}{ }^{\circledR C}$ Claire E.J. Cheetham, ${ }^{1}$ and ${ }^{\oplus N a t h a n i e l ~ N . ~ U r b a n ~}{ }^{1,2,3,4}$ \\ ${ }^{1}$ Department of Biological Sciences, Carnegie Mellon University, Pittsburgh, Pennsylvania $15213,{ }^{2}$ Center for the Neural Basis of Cognition, Pittsburgh, \\ Pennsylvania 15213, and ${ }^{3}$ Department of Neurobiology and ${ }^{4}$ Center for Neuroscience, University of Pittsburgh, Pittsburgh, Pennsylvania 15213
}

In the main olfactory bulb (MOB), the first station of sensory processing in the olfactory system, GABAergic interneuron signaling shapes principal neuron activity to regulate olfaction. However, a lack of known selective markers for MOB interneurons has strongly impeded cell-type-selective investigation of interneuron function. Here, we identify the first selective marker of glomerular layer-projecting deep short-axon cells (GL-dSACs) and investigate systematically the structure, abundance, intrinsic physiology, feedforward sensory input, neuromodulation, synaptic output, and functional role of GL-dSACs in the mouse MOB circuit. GL-dSACs are located in the internal plexiform layer, where they integrate centrifugal cholinergic input with highly convergent feedforward sensory input. GL-dSAC axons arborize extensively across the glomerular layer to provide highly divergent yet selective output onto interneurons and principal tufted cells. GL-dSACs are thus capable of shifting the balance of principal tufted versus mitral cell activity across large expanses of the MOB in response to diverse sensory and top-down neuromodulatory input.

Key words: acetylcholine; chrna2; inhibition; interneuron; olfaction; olfactory bulb

\section{Significance Statement}

The identification of cell-type-selective molecular markers has fostered tremendous insight into how distinct interneurons shape sensory processing and behavior. In the main olfactory bulb (MOB), inhibitory circuits regulate the activity of principal cells precisely to drive olfactory-guided behavior. However, selective markers for MOB interneurons remain largely unknown, limiting mechanistic understanding of olfaction. Here, we identify the first selective marker of a novel population of deep short-axon cell interneurons with superficial axonal projections to the sensory input layer of the MOB. Using this marker, together with immunohistochemistry, acute slice electrophysiology, and optogenetic circuit mapping, we reveal that this novel interneuron population integrates centrifugal cholinergic input with broadly tuned feedforward sensory input to modulate principal cell activity selectively.

\section{Introduction}

The mammalian brain contains a vast diversity of GABAergic inhibitory interneurons. The identification of cell-type-selective molecular markers has fostered tremendous insight into how distinct interneurons shape sensory processing and behavior

\footnotetext{
Received Sept. 13, 2016; revised Nov. 14, 2016; accepted Dec. 3, 2016.

Author contributions: S.D.B. and N.N.U. designed research; S.D.B., G.L., A.L., and C.E.J.C. performed research; S.D.B., G.L., and A.L. analyzed data; S.D.B., G.L., A.L., C.E.J.C., and N.N.U. wrote the paper.

This work was supported by the National Institutes of Health-National Institute on Deafness and Other Communication Disorders (Grant F31DC013490 to S.D.B., Grant R01DC005798 to N.N.U., and Grant R01DC011184 to N.N.U.) and the Pennsylvania Department of Health Commonwealth Universal Research Enhancement Program (N.N.U.). We thank the Mellon Institute Centralized Vivarium staff for excellent technical assistance; Rachel S. Bouchard and Aryn H. Gittis for assistance with Ai3 mice; and Charles R. Gerfen, Laura Kus, and members of the Urban, Gittis, Seal, Kuhlman, and Oswald laboratories for helpful discussions.

The authors declare no competing financial interests.

Correspondence should be addressed to Nathaniel N. Urban, Department of Neurobiology, University of Pittsburgh, E1440 BSTWR, 200 Lothrop Street, Pittsburgh, PA 15213. E-mail: nurban@pitt.edu.

DOI:10.1523/JNEUROSCI.2880-16.2016

Copyright $\odot 2017$ the authors $\quad 0270-6474 / 17 / 371117-22 \$ 15.00 / 0$
}

(Kubota, 2014; Wester and McBain, 2014; Letzkus et al., 2015). In the main olfactory bulb (MOB), inhibitory circuits regulate the activity of principal mitral and tufted cells (M/TCs) precisely to drive olfactory-guided behavior (Fukunaga et al., 2012, 2014; Gschwend et al., 2015). However, selective markers for many MOB interneurons have not been identified (Eyre et al., 2009), impeding investigation of cell-type-selective interneuron function.

Sensory input to the MOB is organized into odorantreceptor-specific glomeruli, where olfactory sensory neuron (OSN) axons and external tufted cells (ETCs) drive sensory activation of $\mathrm{M} / \mathrm{TC}$ apical dendritic tufts in the superficial layers of the MOB (Nagayama et al., 2014). Deeper in the MOB, the inframitral layers contain a large class of GABAergic deep shortaxon cell (dSAC) interneurons, the functional roles of which remain almost entirely unknown. dSACs can be subdivided into granule cell layer (GCL)-, external plexiform layer (EPL)-, and glomerular layer (GL)-projecting dSACs (GCL/EPL/GL-dSACs, 
respectively) (Eyre et al., 2008). GL-dSAC axons arborize across multiple glomeruli and thus may regulate sensory-evoked MOB activity significantly (Eyre et al., 2008). Moreover, GL-dSACs are concentrated in the internal plexiform layer (IPL), a region of dense innervation by ETC and TC axon collaterals (Macrides et al., 1985; Liu and Shipley, 1994), glutamatergic and GABAergic centrifugal feedback (Boyd et al., 2012; Markopoulos et al., 2012; Nunez-Parra et al., 2013), and neuromodulatory input (Macrides et al., 1981; Shipley and Adamek, 1984), and are thus poised to coordinate widespread MOB activity patterns across distinct brain states.

Here, we identify nicotinic acetylcholine receptor (nAChR) subunit $\alpha 2$ (chrna2) as the first selective marker of GL-dSACs. Using Chrna2-Cre mice, we demonstrate that GL-dSACs integrate highly convergent feedforward sensory input from ETCs (and possibly TCs) of several glomeruli and inhibit the apical dendritic tufts of ETCs and TCs selectively across multiple glomeruli. Strikingly, GL-dSACs neither receive MC input nor provide output onto MCs. GL-dSAC activity is also regulated by direct centrifugal cholinergic input. In total, the uniquely divergent yet selective connectivity of GL-dSACs supports a novel interglomerular inhibitory circuit between ETCs and TCs that can be modulated by top-down cholinergic input.

\section{Materials and Methods}

Animals and virus injections. All experiments were completed in compliance with the guidelines established by the Institutional Animal Care and Use Committee of Carnegie Mellon University and the University of Pittsburgh. Chrna2-Cre mice (line OE29) were obtained from GENSAT (Gong et al., 2007) and used as heterozygous F1-2 hybrid crosses (on a mixed FVB/N, Crl:CD1[ICR], and C57BL/6 background) with C57BL/6 and Ai3 mice (Madisen et al., 2010). Approximately one-third of Chrna2-Cre mice exhibited markedly denser Cre-dependent labeling throughout the brain and were not used in this study. For Cre-dependent ChR2:mCherry expression, $\sim 1 \mu$ l of AAV1-EF1a-DIO-hChR2(H134R): mCherry (University of Pennsylvania Vector Core) was injected unilaterally into the MOB or forebrain at postnatal day 2 (P2)-P3 (Cheetham et al., 2015). For Cre-dependent EGFP expression, $\sim 1 \mu \mathrm{l}$ of AAV1-CAGflex-GFP (University of North Carolina Vector Core) was injected unilaterally into the adult MOB.

Immunohistochemistry. Mice were anesthetized with intraperitoneal injection of $0.4 \mathrm{ml}$ of ketamine $(20 \mathrm{mg} / \mathrm{ml}) / x y l a z i n e ~(3.3 \mathrm{mg} / \mathrm{ml})$ and then transcardially perfused with $1 \% \mathrm{NaCl}$ in $0.1 \mathrm{M}$ phosphate buffer (PB) followed by paraformaldehyde ( $4 \%$ in PB). Brains were extracted, postfixed in paraformaldehyde overnight, sunk in sucrose (30\% w/v in PB), and then cryogenically sliced into $25-\mu \mathrm{m}$-thick sagittal sections with a sliding microtome (Leica, SM2000R). Sections were incubated with Triton X-100 $(0.1 \%$ in $\mathrm{PB})$ and normal donkey serum (NDS; $2 \%$ in $\mathrm{PB})$ for $1 \mathrm{~h}$ at room temperature, washed with $\mathrm{PB}$, and then incubated for $1 \mathrm{~h}$ at room temperature (unless otherwise noted) with combinations of the following primary antibodies (in PB with 2\% NDS and $0.05 \%$ Tween 20): goat anti-GFP (Abcam, ab6673; 1:10000), rabbit anti-GFP (Invitrogen, A-11122; 1:1000), rabbit anti-GABA ${ }_{\mathrm{A}} \mathrm{R} \alpha 1$ (Alomone Labs, AGA-001; 1:1000), rabbit anti-Na $\mathrm{N}_{\mathrm{V}} 1.6$ (Abcam, ab83764; 1:2000; overnight incubation at 4C), sheep anti-tyrosine hydroxylase (TH) (EMD Millipore, AB1542; 1:1000), mouse anti-PV (clone PARV-19; Sigma, P3088; 1:1000; overnight incubation at 4C), rat antim2AChR (clone M2-2-B3; EMD Millipore, MAB367; 1:500), goat anti-SST (Santa Cruz Biotechnology, sc-7819; 1:500; overnight incubation at 4C), goat anti-OMP (Wako, 544-10001; 1:5000; overnight incubation at 4C), and goat anti-CR (Santa Cruz Biotechnology, sc-11644; 1:1500). Sections were then washed in $\mathrm{PB}$ and incubated for $1 \mathrm{~h}$ at room temperature with combinations of the following secondary antibodies raised in donkey (1:600 in PB with 2\% NDS and 0.05\% Tween 20): anti-goat AF488, anti-rabbit AF488, anti-rabbit AF594, anti-sheep AF594, anti-mouse AF594, anti-goat AF594, anti-rat AF594 (Invitrogen, A-11055, A-21206, A-21207, A-11016, A-21203, A-11058, A-21209, respectively).
For chromogenic Neurobiotin labeling, acute slices containing Neurobiotin-filled cells were fixed in paraformaldehyde (4\% in PB) for $>24 \mathrm{~h}$ at $4 \mathrm{C}$, quenched with $\mathrm{H}_{2} \mathrm{O}_{2} /$ methanol $(1 \% / 10 \%$ in $\mathrm{PB})$ for $1 \mathrm{~h}$ at room temperature, washed with $\mathrm{PB}$, permeabilized with Triton X-100 ( $2 \%$ in $\mathrm{PB})$ for $1 \mathrm{~h}$ at room temperature, and then incubated overnight at $4 \mathrm{C}$ in Vectastain $\mathrm{ABC}$ complex solution (Vector Laboratories; 1\% Reagent A, $1 \%$ Reagent $B$ in PB with $1 \%$ Triton $X-100$ ). Slices were then washed extensively with $\mathrm{PB}$ before incubation with $\mathrm{DAB}$ (Sigma-Aldrich; $0.5 \mathrm{mg} / \mathrm{ml}$ with $0.01 \% \mathrm{H}_{2} \mathrm{O}_{2}$ in $\mathrm{PB}$ ) for $10 \mathrm{~min}$ at room temperature.

Imaging. Immunofluorescent images were collected on inverted Zeiss LSM 510 META Duoscan and 880 confocal microscopes using 40-100 $\times$ oil-immersion objectives in maximum intensity projections of $0.5 \mu \mathrm{m}$ step $z$-stacks at $2048 \times 2048$ resolution. Chrna2-Cre-labeled dSAC abundance was calculated by counting Chrna2-Cre-labeled dSACs within manually drawn IPL and GCL borders across stitched $10 \times 2 \mu \mathrm{m}$-step $z$-stack tile-scans of the entire MOB. Three sagittal sections were imaged per animal and used to evaluate mediolateral bias and calculate a total cell density and abundance per animal. Dorsoventral bias was evaluated by comparing total cell densities within dorsal and ventral halves of each of the three MOB tile scans per animal. $\mathrm{GABA}_{\mathrm{A}} \mathrm{R} \alpha 1$-expressing $\mathrm{dSAC}$ abundance was calculated by counting $\mathrm{GABA}_{\mathrm{A}} \mathrm{R} \alpha 1$-positive dSACs within manually drawn IPL and GCL borders across 5-6 random-field $40 \times 0.5 \mu \mathrm{m}$-step $z$-stacks per animal, calculating total IPL- and GCLspecific cell densities per animal and then averaging across animals, similar to previous methods (Eyre et al., 2009). For DAB-stained tissue and whole-brain imaging, images were collected on an Olympus BX51WI microscope using $10-20 \times$ air objectives and a $100 \times$ oil-immersion objective.

Slice preparation. $\mathrm{P} 18-\mathrm{P} 28$ mice of both sexes were anesthetized with isoflurane and decapitated into ice-cold oxygenated dissection solution containing the following (in $\mathrm{mm}$ ): $125 \mathrm{NaCl}, 25$ glucose, $2.5 \mathrm{KCl}, 25$ $\mathrm{NaHCO}_{3}, 1.25 \mathrm{NaH}_{2} \mathrm{PO}_{4}, 3 \mathrm{MgCl}_{2}$, and $1 \mathrm{CaCl}_{2}$. Brains were isolated and acute horizontal or sagittal slices ( $310 \mu \mathrm{m}$ thick) were prepared using a vibratome (5000mz-2; Campden or VT1200S; Leica). Slices recovered for $30 \mathrm{~min}$ in $\sim 37^{\circ} \mathrm{C}$ oxygenated Ringer's solution that was identical to the dissection solution except with lower $\mathrm{Mg}^{2+}$ concentrations (1 mM $\mathrm{MgCl}_{2}$ ) and higher $\mathrm{Ca}^{2+}$ concentrations $\left(2 \mathrm{mM} \mathrm{CaCl}_{2}\right)$. Slices were then stored at room temperature until recording.

Electrophysiology. Slices were continuously superfused with $34^{\circ} \mathrm{C}$ oxygenated Ringer's solution. Cells were visualized using infrared differential interference contrast video microscopy. Current-clamp recordings were made using electrodes (4-9 M $\Omega$ ) filled with the following (in $\mathrm{mM}$ ): $120 \mathrm{~K}$-gluconate, $2 \mathrm{KCl}, 10 \mathrm{HEPES}, 10 \mathrm{Na}$-phosphocreatine, $4 \mathrm{Mg}$-ATP, $0.3 \mathrm{Na}_{3} \mathrm{GTP}, 0.2 \mathrm{EGTA}$, and 0.025 AF594, along with $0.2 \%$ Neurobiotin (Vector Labs). Voltage-clamp recordings were made using electrodes filled with the following (in mM): 140 Cs-gluconate, $10 \mathrm{QX}-314,2 \mathrm{KCl}, 10$ HEPES, $10 \mathrm{Na}$-phosphocreatine, $4 \mathrm{Mg}$-ATP, $0.3 \mathrm{Na}_{3} \mathrm{GTP}$, and 0.025 AF594, along with $0.2 \%$ Neurobiotin. Liquid junction potentials were $12-14 \mathrm{mV}\left(\mathrm{K}^{+}\right.$-internal $)$and $11 \mathrm{mV}\left(\mathrm{Cs}^{+}\right.$-internal $)$and were not corrected for except where noted. Data were low-pass filtered at $4 \mathrm{kHz}$ and digitized at $10 \mathrm{kHz}$ using a MultiClamp 700A amplifier (Molecular Devices) and an ITC-18 acquisition board (Instrutech) controlled by custom software written in IGOR Pro (WaveMetrics). OSN fiber bundles projecting into single glomeruli were stimulated (100- $\mu$ s-long constant current pulses) using an extracellular monopolar glass electrode. For optogenetic stimulation, slices were illuminated by a $75 \mathrm{~W}$ xenon arc lamp passed through a YFP filter set and $60 \times$ water-immersion objective centered on the recorded cell (GL-dSACs) or the GL (all other cells). NBQX (Tocris Bioscience and Sigma-Aldrich), AP5 (Tocris Bioscience), GBZ (Tocris Bioscience), DMPP (Alomone Labs), and Mec (Tocris Bioscience and Alomone Labs) were added to the bath or focally applied as indicated. Cell morphologies were reconstructed under a $100 \times$ oilimmersion objective and analyzed with Neurolucida software (MBF Bioscience). Reconstructed GL-dSAC dendrites and axons are drawn in black and red, respectively. Gray lines in reconstructions denote GL/EPL, EPL/MCL, MCL/IPL, and IPL/GCL borders.

Data analysis. Synaptic events were detected and analyzed in Axograph. Synaptic events were detected with a $1 \mathrm{~ms}$ minimum event separation time. sEPSCs were detected using a 4.5-ms-long double expo- 
nential template with $0.5 \mathrm{~ms}$ baseline, $0.4 \mathrm{~ms}$ rise time constant, and $2 \mathrm{~ms}$ decay time constant. sIPSCs were detected using a 21-ms-long double exponential template with $1 \mathrm{~ms}$ baseline, $0.6 \mathrm{~ms}$ rise time constant, and 8 ms decay time constant. All events were detected with a threshold amplitude of $3 \times \mathrm{SD}$ of the baseline noise. Event amplitudes, 20-80\% rise times, and decay constants were calculated using Axograph. Extracellular stimulation-evoked synaptic latencies were defined as the interval of time from extracellular stimulation to the onset of the synaptic response, which was defined as the time at which the synaptic response reached 5\% of its peak amplitude. Photostimulation-evoked synaptic latencies were defined as the median photostimulation-evoked GL-dSAC first-spike latency (see Fig. 11H) subtracted from the absolute synaptic latency (calculated as above). Synaptic input durations were defined as the interval of time from the onset of the synaptic response to the time at which the synaptic response decayed to $5 \%$ of its peak amplitude. For paired GL-dSAC/MC recordings, charge transferred was calculated by integrating current traces from the end of the stimulus artifact to $1 \mathrm{~s}$ or $50 \mathrm{~ms}$ after OSN stimulation for MCs and GL-dSACs, respectively. For all other recordings, charge transferred was calculated by integrating current traces from the onset of the synaptic response to the time at which the synaptic response decayed to 5\% of its peak amplitude. Stimulus artifacts are blanked for visual clarity of synaptic responses. Intrinsic biophysical properties were calculated as described previously (Burton and Urban, 2014 , 2015) using a $20 \mathrm{mV} / \mathrm{ms}$ threshold for detecting action potentials. To calculate power spectral densities from spontaneous firing epochs, binary spike trains were converted to sinusoidal spike phases and analyzed using Welch's method (500 ms windows, 50\% window overlap). Values are reported as mean \pm SD. Error bars and shading denote mean \pm SEM. Normally and non-normally distributed data (AndersonDarling test) were analyzed using parametric and non-parametric tests, respectively, as indicated.

\section{Results}

\section{Targeting GL-dSACs genetically}

GL-dSACs are concentrated in the MOB IPL and superficial GCL (sGCL) (Fig. 1A) and are composed of horizontal cells and a subset of stellate Golgi cells (Eyre et al., 2008). To study GLdSACs systematically, we therefore searched for candidate markers that are expressed in IPL/sGCL-located cells selectively. In situ hybridization of chrna2 labels sparse IPL/sGCL-located cells within the MOB (Ishii et al., 2005). Consistent with this previous report, localized AAV injection in the adult MOB of transgenic Chrna2-Cre mice (Fig. 1B) yielded highly selective Credependent EGFP expression in multipolar neurons within the IPL/sGCL (Fig. 1C). Moreover, putative axons in the GL extended up to $\sim 1 \mathrm{~mm}$ away from the nearest EGFP-expressing soma (Fig. 1C), matching the extensive axonal arborizations observed previously for GL-dSACs (Eyre et al., 2008).

Compared with adult AAV injection, constitutive Chrna2Cre/Ai3 reporter crosses (Fig. 1B) yielded less selective labeling, with a small fraction of M/TCs, GCs, and non-MOB cells expressing EYFP (Fig. 2A-G; see Materials and Methods). However, EYFP expression was still heavily concentrated in IPL/sGCLlocated dSACs throughout the MOB (Fig. 2A,B,G). Quantification of labeled cell densities yielded 282.2 \pm 92.6 IPL-located $\mathrm{dSACs} / \mathrm{mm}^{3}$ (316 cells counted, $n=4$ mice) and $147.4 \pm 63.6$ sGCL-located dSACs $/ \mathrm{mm}^{3}$ (174 cells counted, $n=4$ mice) across the entire MOB volume, with no dorsoventral or mediolateral bias (Fig. $2 \mathrm{H}-\mathrm{J}$ ). Applying previous volumetric measures (Parrish-Aungst et al., 2007), we therefore estimate that adult Chrna2-Cre mice label (i.e., drive Cre-dependent expression in) $1797.9 \pm 589.6$ IPL-located dSACs, $939.1 \pm 404.8$ sGCL-located dSACs, and $2736.9 \pm 765.8$ total dSACs per MOB.

The total number of dSACs in the adult mouse MOB is not currently known, though Nusser and colleagues have used the specific but nonselective expression of $\mathrm{GABA}_{\mathrm{A}} \mathrm{R} \alpha 1$ in all deep
GCL-located dSACs and $~ 50 \%$ of IPL/sGCL-located dSACs to estimate $\sim 13,500$ total dSACs in the adult rat MOB (Eyre et al., 2009). Therefore, to determine what fraction of total dSACs that Chrna2-Cre mice label, we quantified the number and colocalization of Chrna2-Cre-labeled and $\mathrm{GABA}_{\mathrm{A}} \mathrm{R} \alpha 1$-labeled dSACs in adult Chrna2-Cre/Ai3 mice. Using previous volumetric measures (Parrish-Aungst et al., 2007), $\mathrm{GABA}_{\mathrm{A}} \mathrm{R} \alpha 1$ was moderately to strongly expressed in 2706.0 \pm 405.4 IPL-located dSACs, 7558.9 \pm 962.4 GCL-located dSACs (both sGCL and deep GCL), and 10,231.1 \pm 909.2 total dSACs per MOB (193 cells counted, $n=3$ mice) (Fig. $1 D$ ). Consistent with the negligible to weak expression of $\mathrm{GABA}_{\mathrm{A}} \mathrm{R} \alpha 1$ in $\sim 50 \%$ of IPL/sGCL-located dSACs (Eyre et al., 2009) and the restricted localization of Chrna2-Crelabeled dSACs to the IPL/sGCL, only $53.7 \%$ of Chrna2-Crelabeled dSACs (29/54 cells) exhibited detectable $\mathrm{GABA}_{\mathrm{A}} \mathrm{R} \alpha 1$ expression (Fig. 1D). Assuming that Chrna2-Cre-labeled dSACs comprise the entire population of $\mathrm{GABA}_{\mathrm{A}} \mathrm{R} \alpha 1$-negative dSACs, we therefore estimate that the adult mouse MOB contains $\sim 3500$ IPL-located dSACs, $\sim 8000$ GCL-located dSACs, and $\sim 11,500$ total dSACs, of which $\sim 51 \%$ located in the IPL and $\sim 12 \%$ located in the GCL are labeled selectively in Chrna2-Cre mice.

To better resolve the morphologies of Chrna2-Cre-labeled dSACs, we filled individual EYFP-expressing dSACs with Neurobiotin via targeted whole-cell recordings in acute slices from Chrna2-Cre/Ai3 mice (Fig. $3 A, B$ ). Post hoc reconstruction of a large subset (Fig. $3 C, D$ ) revealed that Chrna2-Cre-labeled dSACs extend $4.7 \pm 1.5(n=24)$ sparsely spiny and beaded dendrites up to $\sim 200 \mu \mathrm{m}$ through the IPL parallel to the MCL and frequently project a single thin putative axon superficially to arborize across the GL (Fig. 3E, Table 1). Importantly, we never observed a putative axon arborize across the EPL or GCL or enter the lateral olfactory tract, though we note that the processes of some cells were truncated proximal to the soma and could not be traced. In addition, processes entering the GL could only be traced a short distance due to their thin, tortuous profile and the high cell density of the GL. Chrna2-Cre-labeled dSAC morphologies nevertheless closely match the GL-dSAC morphologies recovered previously from serial thin section reconstruction (Eyre et al., 2008). Moreover, high densities of axon initial segment channel $\mathrm{Na}_{\mathrm{V}} 1.6$ (Lorincz and Nusser, 2008) within the proximal regions of the superficial processes of Chrna2-Cre-labeled dSACs (Fig. $1 F$ ) confirmed that Chrna2-Cre-labeled dSACs project their axons superficially into the GL. Chrna2-Cre-labeled somata likewise poorly colocalized with nonselective markers of EPL- and GCL-dSACs (Fig. 4) and Chrna2-Cre-labeled axonal boutons in the GL poorly colocalized with superficial short-axon cell marker TH (Fig. 1E).

Collectively, our results thus show that Chrna2-Cre mice label GL-dSACs selectively in the MOB, establishing the first selective marker of any dSAC subclass. Further, across 158 untargeted recordings, Nusser and colleagues previously observed that GLdSACs constitute $\sim 59 \%$ and $\sim 18 \%$ of IPL- and GCL-located dSACs, respectively (Eyre et al., 2008), fractions closely corresponding to the $\sim 51 \%$ and $\sim 12 \%$ of IPL- and GCL-located dSACs labeled selectively in Chrna2-Cre mice. Our results thus further suggest that Chrna2-Cre mice label most, if not all, GL-dSACs.

\section{GL-dSACs fire intrinsically at theta frequencies}

Cell-attached recordings from GL-dSACs in acute slices (Fig. 5A) revealed spontaneous firing at $\sim 6 \mathrm{~Hz}$ that was highly regular and stable, yielding robust spectral peaks at $5-20 \mathrm{~Hz}$ (Fig. $5 \mathrm{~B}, C$ ) and low interspike interval (ISI) coefficient of variation $\left(\mathrm{CV}_{\mathrm{ISI}}\right)$ values 
A

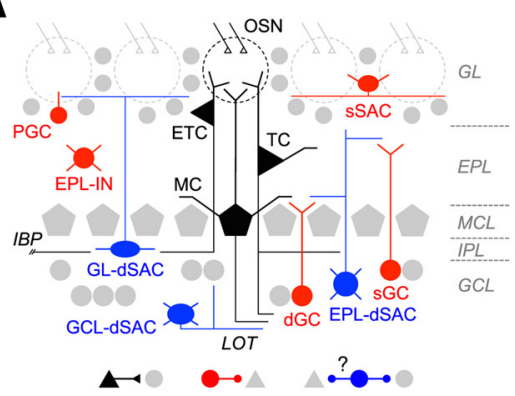

B

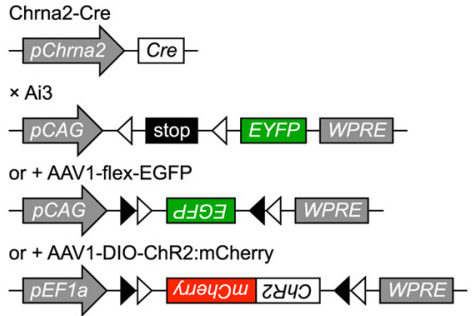

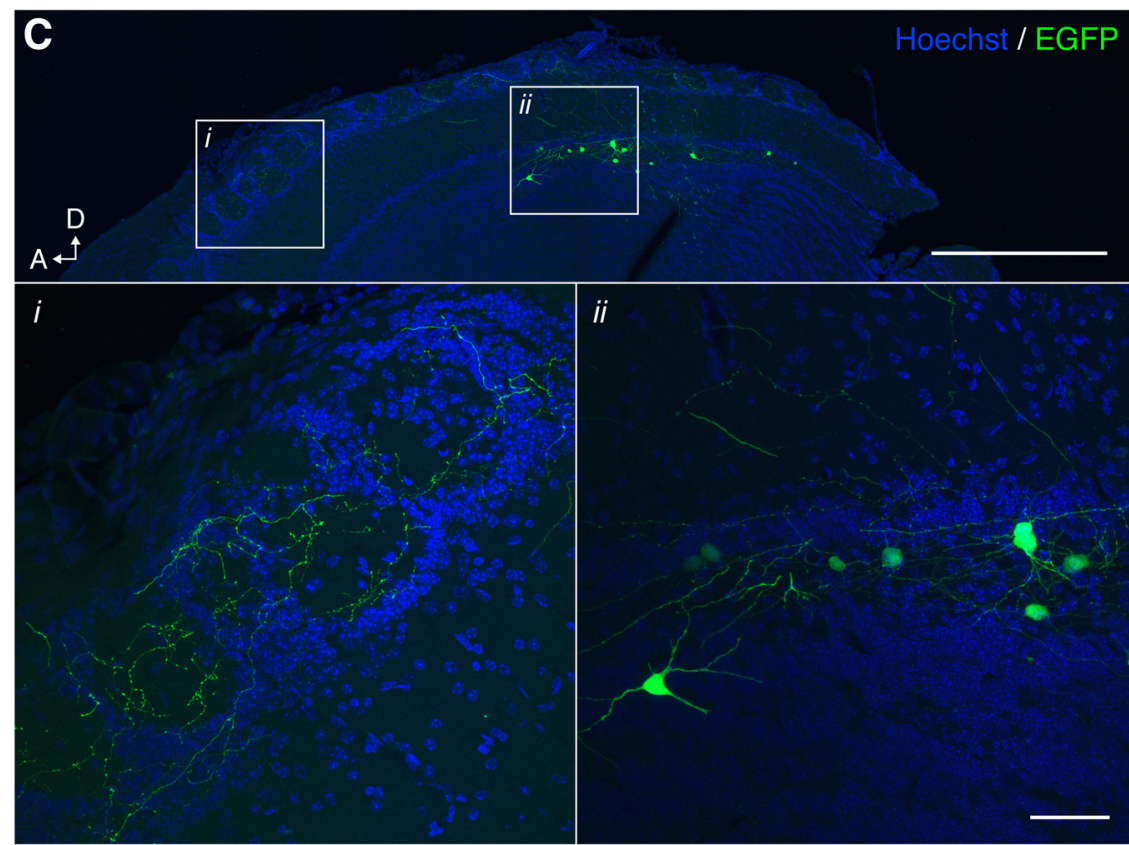
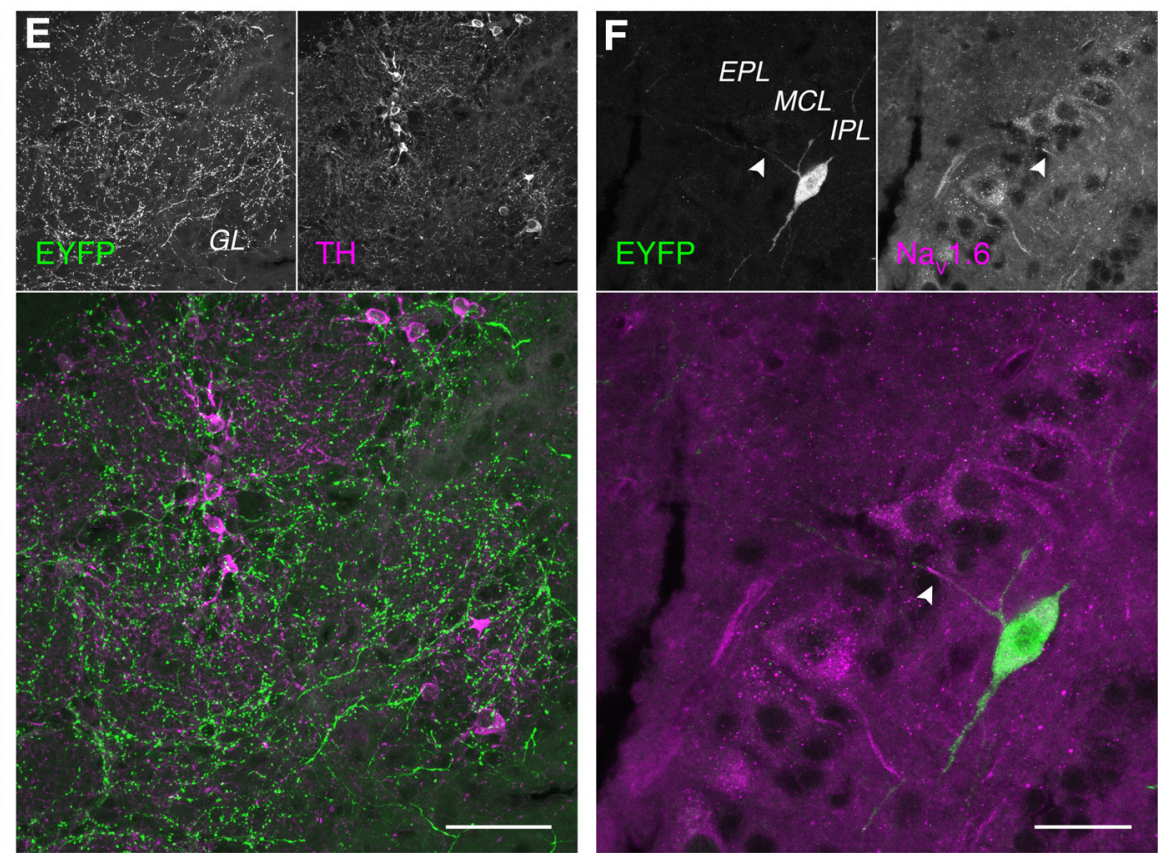

Figure 1. Chrna2-Cre mice label GL-dSACs selectively. $A, M O B$ circuitry. Glutamatergic cells are shown in black. GABAergic cells targeting glutamatergic cells are shown in red. GABAergic cells putatively targeting other GABAergic cells are shown in blue. $\boldsymbol{B}$, Experimental strategy to drive Cre-dependent expression of EYFP, EGFP, or ChR2:mCherry under the control of the chrna2 promoter. C, Cre-dependent expression of EGFP 2 weeks after AAV injection into the adult dorsal MOB. Scale bar, $500 \mu \mathrm{m}$ (inset: $50 \mu \mathrm{m}$ ). A, Anterior; D: dorsal. D, Half of the Chrna2-Cre-labeled GL-dSACs (46.3\%; $25 / 54$ cells, $n=2$ mice) exhibit weak $G_{A B A} R \alpha 1$ expression (arrowheads), whereas the remaining cells exhibit no or negligible GABA $R \alpha 1$ expression (arrow). Scale bar, $50 \mu m$. $\boldsymbol{E}$, Chrna2-Cre-labeled axonal boutons colocalize poorly with TH. Scale bar, $50 \mu \mathrm{m}$. $\boldsymbol{F}, \mathrm{Na}_{\mathrm{v}} 1.6$ localizes to proximal regions of superficially projecting processes of Chrna2-Cre-labeled dSACs (arrowhead). Scale bar, $20 \mu \mathrm{m}$.

(Table 2). Application of NBQX (10 $\mu \mathrm{M})$, AP5 $(50 \mu \mathrm{M})$, and gabazine (GBZ; $10 \mu \mathrm{M}$ ) had no effect on spontaneous firing rates (Fig. $5 B, C$ ) or regularity (Table 2), demonstrating that highly regular firing is an intrinsic property of GL-dSACs. Consistent with this finding, whole-cell recordings from a large population of GL-dSACs $(n=33)$ likewise revealed highly regular and stable firing at $\sim 8 \mathrm{~Hz}$ (Fig. 5D-F, H,I, Table 2).

Spontaneous firing was abolished by moderate hyperpolarization from a mean membrane potential $\left(V_{\mathrm{m}}\right)$ measured during ISIs $\left(V_{\mathrm{m}, \mathrm{ISI}}\right)$ of $-55.0 \pm 3.0 \mathrm{mV}$ to $-61.6 \pm 2.7 \mathrm{mV}(n=20)$, enabling examination of GL-dSAC excitability through somatic step current injections. The majority of GL-dSACs responded to depolarizing current with highly regular firing (Fig. 5G,K) and relatively uniform excitability (Fig. $5 L$, Table 2). Surprisingly, whereas all GL-dSACs exhibited regular firing at rest (Fig. $5 F, I$ ), $23.1 \%$ (6/26 cells) responded to weak depolarizing current $(<50$ pA) from hyperpolarized $\mathrm{V}_{\mathrm{m}}$ with prominent $3-4 \mathrm{~Hz}$ burst firing (Fig. $5 J$ ), whereas stronger currents $(>50 \mathrm{pA})$ evoked highly regular firing (Fig. $5 \mathrm{~K}$ ). Likewise, hyperpolarizing currents evoked rebound bursting (Fig. 5J) in 39.1\% (9/23 cells) of GL-dSACs. 

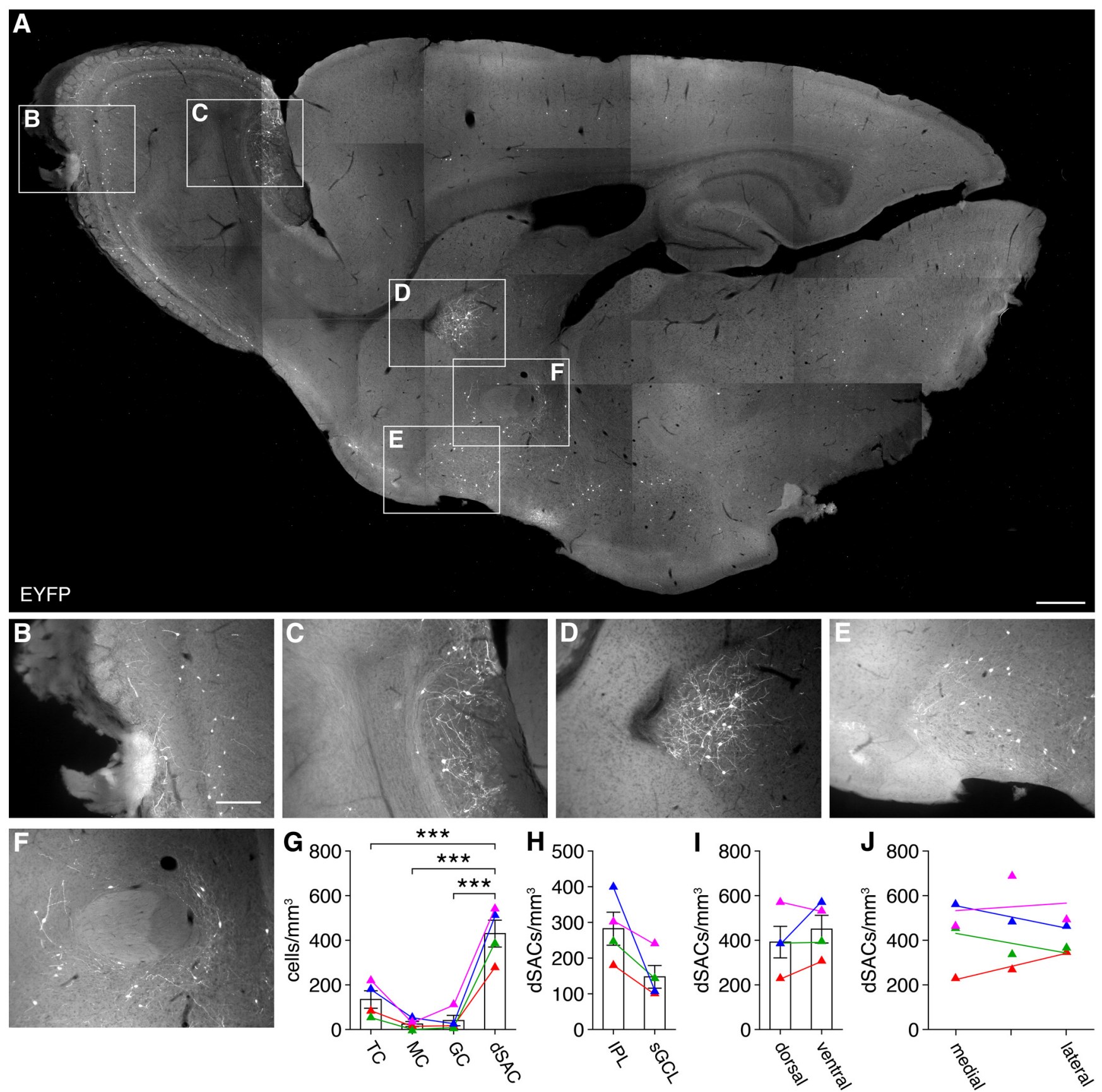

Figure 2. Characterization of the transgenic Chrna2-Cre mouse line. $\boldsymbol{A}-\boldsymbol{F}$, Sagittal section from a representative Chrna2-Cre/Ai3 mouse. Within the MOB, EYFP expression is localized to dSACs in the IPL and to sparse M/TCs and GCS (B). Outside of the MOB, EYFP expression is largely localized to sparse neurons in the accessory olfactory bulb $(\boldsymbol{C})$, septal nucleus (D), and multiple basal forebrain regions, including the ventral pallidum, bed nucleus stria terminalis, and substantia innominata $(\boldsymbol{E}, \boldsymbol{F})$. Scale bar, $500 \mu \mathrm{m}$ (insets: $200 \mu \mathrm{m}$ ). $\boldsymbol{G}$, Chrna2-Cre/Ai3 mice labeled significantly more dSACs than other cell types in the MOB $\left(429.7 \pm 120.2, n=4\right.$, vs $134.3 \pm 78.5, n=4$, vs $24.2 \pm 22.5, n=4$, vs $40.0 \pm 46.3, n=4$, cells $/ \mathrm{mm}^{3}$, dSACs vs TCs vs MCs vs GCs; $p=2.2 \times 10^{-5}$, one-way ANOVA; dSACs vs TCS, $p=7.1 \times 10^{-4}$, dSACs vs MCs, $p=3.6 \times 10^{-5}$, dSACs vs GCs, $p=5.4 \times 10^{-5}$, post hoc Tukey-Kramer). Colors correspond to different Chrna2-Cre/Ai3 mice. $\boldsymbol{H}$, Chrna2-Cre/Ai3 mice labeled dSACs within the IPL and sGCL, with a strong trend toward greater labeled dSAC density in the IPL ( $p=0.08$, paired two-sided $t$ test). I, J, Chrna2-Cre/Ai3-labeled dSACS were equally distributed along dorsoventral $(I ; p=0.34$, paired two-sided $t$ test) and mediolateral $(J ; p=0.87$; two-sided $t$ test of linear regression slopes) axes of the MOB.

Collectively, this state-dependent bursting closely matches recent characterization of rat Golgi cells (Pressler et al., 2013) and suggests that GL-dSACs may comprise distinct functional and/or morphological subtypes (e.g., regular firing horizontal cells and bursting Golgi cells).

To evaluate the heterogeneity of GL-dSACs, we subdivided GL-dSACs into regular firing or bursting cells based on their above response to depolarizing and hyperpolarizing step current injections and performed a series of multivariate physiological and morphological analyses. First, direct comparison of 18 physiological properties (Table 3) and 13 morphological properties (Table 4) across the two subpopulations revealed overall minor differences, with bursting cells exhibiting smaller afterhyperpolarizations, more regular spontaneous firing, greater total dendritic length, and more dendritic branch points. Second, principal component analysis across the 18 physiological properties (Fig. 5M-O) and 13 morphological properties (Fig. $3 F-H$ ) revealed no obvious clustering of regular firing and bursting cells. 

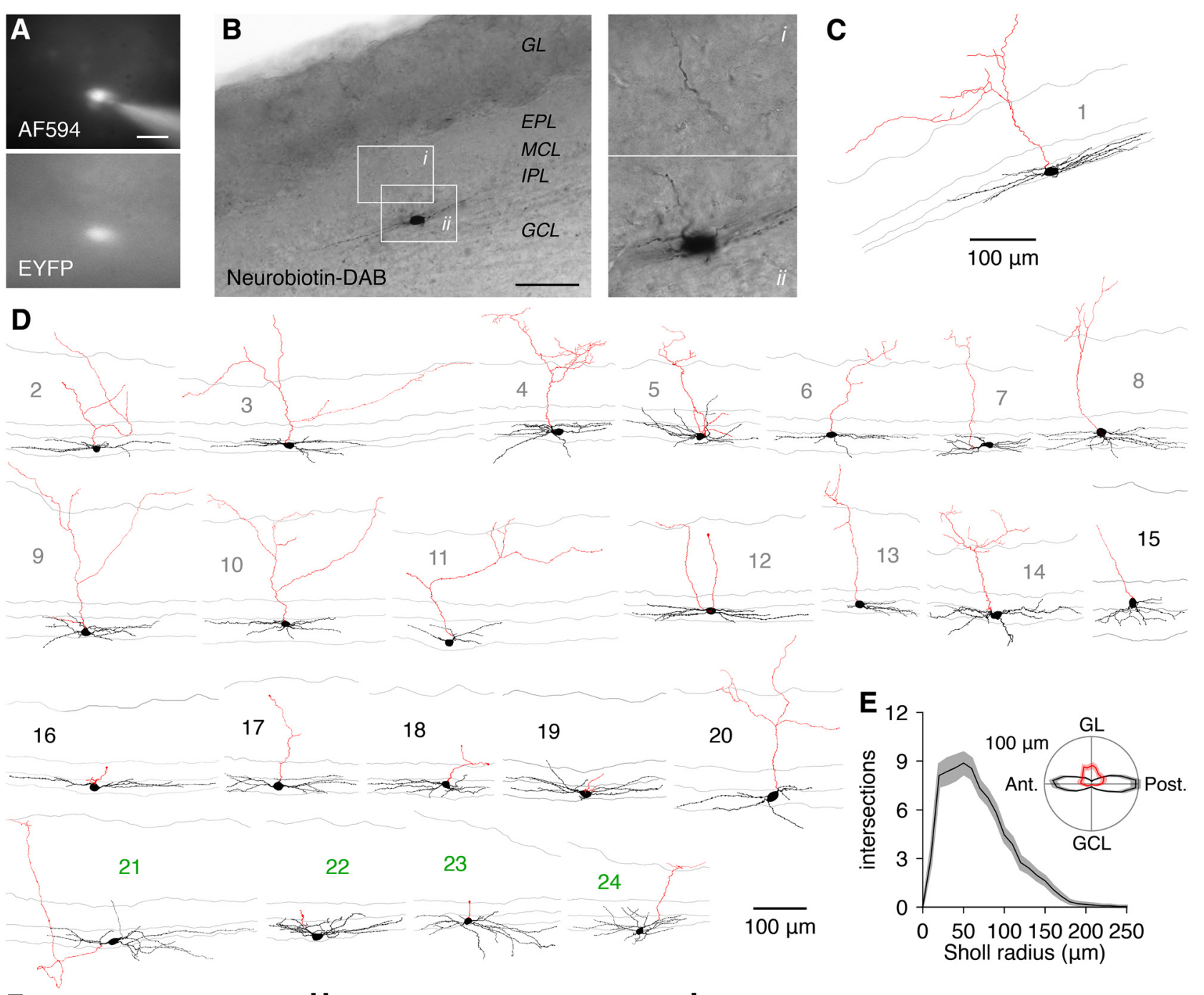

18
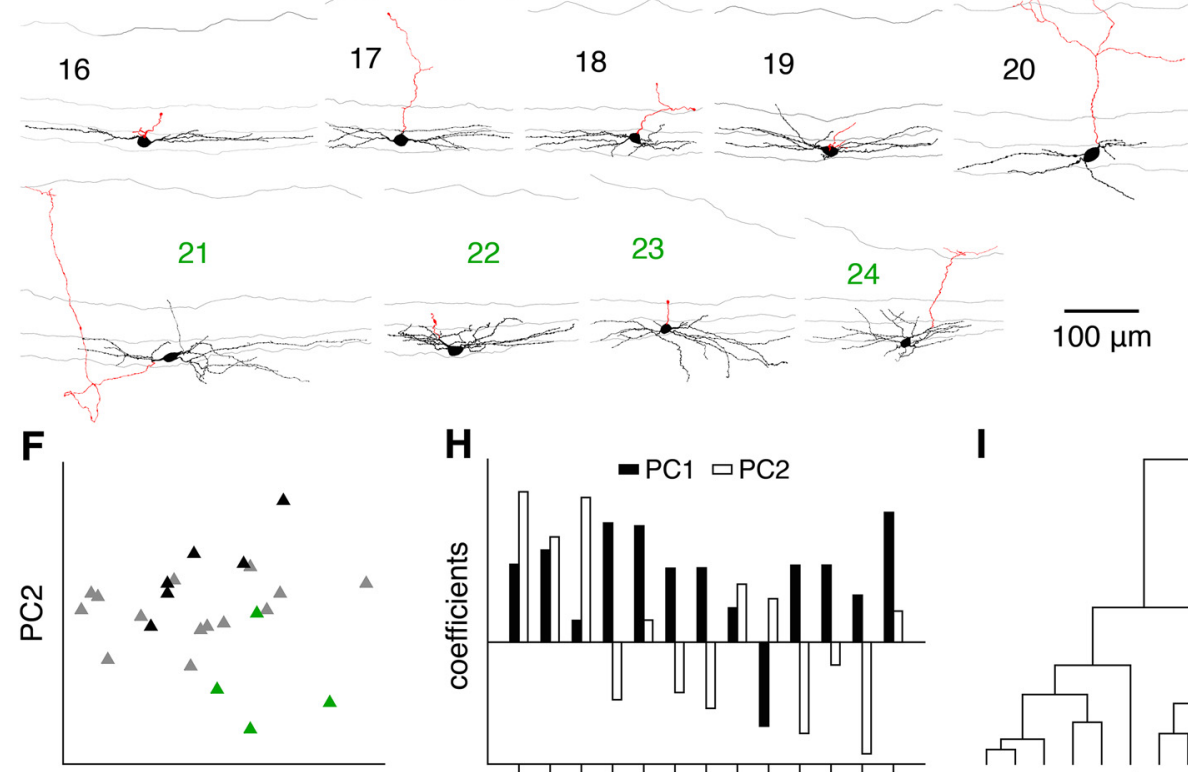

I
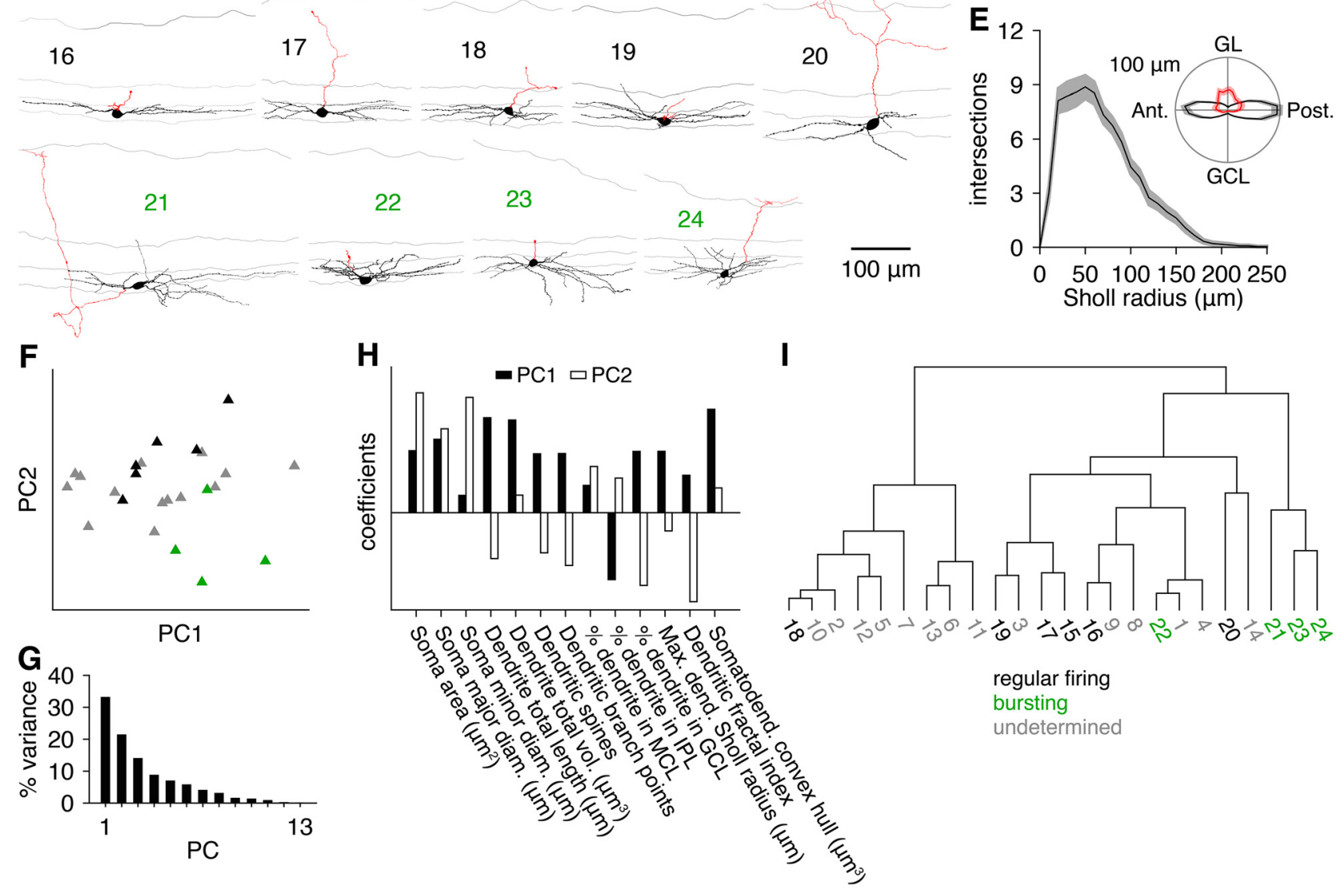

Figure 3. Morphological properties of GL-dSACS. $\boldsymbol{A}, \boldsymbol{B}$, EYFP-expressing dSACs in Chrna2-Cre/Ai3 mice were filled with AF594 and Neurobiotin via whole-cell recordings. Insets show axon projecting through the EPL and into the GL. Scale bar, $20 \mu \mathrm{m}(\boldsymbol{A}) ; 100 \mu \mathrm{m}(\boldsymbol{B})$. $\boldsymbol{C}$, Reconstructed morphology of the cell shown in $\boldsymbol{A}$ and $\boldsymbol{B}$. Scale is the same as in $\boldsymbol{B}$. $\boldsymbol{D}$, Additional reconstructions, sorted by firing pattern. Gray numbers mark GL-dSACs in which the firing pattern was undetermined; black numbers mark regularly firing GL-dSACs; green numbers mark bursting GL-dSACs. $\boldsymbol{E}$, Dendritic Sholl analysis. Inset, Mean angular distribution of reconstructed dendritic and axonal process lengths showing horizontal dendritic extensions (black) and superficial axonal projections (red) $(n=24)$. F, Projection of regular firing (black) and bursting (green) GL-dSACs and GL-dSACs with undetermined firing patterns (gray) onto the first two principal components (PC1 and PC2) identified from principal component analysis of GL-dSAC morphological properties. No obvious clustering of GL-dSACs by firing pattern emerged. G, Variance in GL-dSAC morphological properties explained by each principal component. $\boldsymbol{H}$, Decomposition of PC1 and PC2 into their constituent property coefficients. $\boldsymbol{I}$, Unbiased agglomerative hierarchical clustering of GL-dSACs by the Euclidean distances among the $\boldsymbol{z}$-scored morphological properties listed in $\boldsymbol{H}$ (using Ward's method). Application of the gap statistic method yielded one cluster. 
Table 1. Morphological GL-dSAC properties

\begin{tabular}{lc}
\hline Soma & \\
Area $\left(\mu \mathrm{m}^{2}\right)$ & $227.7 \pm 46.0(196.2-257.2)(24)$ \\
Major diameter $(\mu \mathrm{m})$ & $21.2 \pm 3.0(19.1-22.7)(24)$ \\
Minor diameter $(\mu \mathrm{m})$ & $14.5 \pm 1.6(13.3-15.0)(24)$ \\
Dendrite & \\
Total length $(\mu \mathrm{m})$ & $1150.3 \pm 349.1(876.4-1431.0)(24)$ \\
Total volume $\left(\mu \mathrm{m}^{3}\right)$ & $699.7 \pm 342.1(465.4-849.7)(24)$ \\
Spines & $6.0 \pm 6.3(2.0-6.5)(24)$ \\
Branch points & $9.0 \pm 3.6(7.0-11.0)(24)$ \\
Length in $\mathrm{MCL}(\%)$ & $8.7 \pm 9.9(0.5-11.7)(24)$ \\
Length in IPL $(\%)$ & $80.4 \pm 17.5(70.8-94.9)(24)$ \\
Length in $\mathrm{GCL}(\%)$ & $10.9 \pm 14.6(1.2-16.9)(24)$ \\
Maximum Sholl radius $\left(\mu \mathrm{m}^{3}\right)$ & $161.3 \pm 37.9(140.0-180.0)(24)$ \\
Fractal index & $1.07 \pm 0.03(1.05-1.09)(24)$ \\
Somatodendritic & \\
Convex hull volume $\left(\times 10^{5} \mu \mathrm{m}^{3}\right)$ & $3.5 \pm 2.3(1.9-3.9)(24)$ \\
\hline Values are shown as mean $\pm \mathrm{SD}\left(\mathrm{Q}_{1}-\mathrm{Q}_{3}\right)(\mathrm{n})$. &
\end{tabular}

Finally, unbiased agglomerative hierarchical clustering of GLdSACs by either the 18 physiological properties (Fig. $5 P$ ) or 13 morphological properties (Fig. 3I) and application of the gap statistic method consistently yielded a single cluster of cells. It is possible that an insufficient number of neurons were recorded to resolve multiple clusters statistically across 13-18 properties. However, repeating the agglomerative hierarchical clustering analysis using the first two to four principal components derived from either the 18 physiological properties or 13 morphological properties, as performed previously to differentiate between GCL-, EPL-, and GL-dSACs (Eyre et al., 2008), likewise yielded a single cluster of cells (data not shown). These results thus strongly argue that GL-dSACs comprise a single functional and morphological class, with regular firing and bursting GL-dSACs and horizontal and Golgi cell morphologies representing distinct ends of a continuum.

\section{GL-dSACs receive highly convergent ETC-mediated excitation}

Under baseline conditions, GL-dSACs are continuously bombarded with spontaneous excitatory and inhibitory postsynaptic currents (sEPSCs and sIPSCs, respectively) (Figs. $6 B-D, 7 A, B$, Tables 2, 3), identifying the IPL as a region of high synaptic activity. Physiological activation of a single nearby glomerulus via extracellular stimulation of OSN fiber bundles (Fig. 6A), analogous to the presentation of a low concentration odor in vivo (Mainland et al., 2014), evoked robust, short-latency excitatory input to GL-dSACs that was graded across stimulation intensities (Fig. $6 E-H$ ). This robust excitatory input was observed regardless of whether the GL-dSAC axon entered the GL or was truncated in the EPL during slice preparation (Fig. $6 R-U$ ), excluding the possibility of direct OSN input to GL-dSAC axons within the GL. Excitatory input to GL-dSACs after glomerular activation was also reliably followed by feedforward inhibition (Fig. 7C-F).

The excitatory input evoked by glomerular activation reliably triggered one or two short-latency action potentials in GLdSACs, followed by a consistent $\sim 100 \mathrm{~ms}$ pause in firing (Fig. $6 J-Q$ ). This temporal response (rather than a change in firing rate) was likewise observed across trains of OSN stimulation at 5 and $10 \mathrm{~Hz}$ (Fig. 8), frequencies mimicking physiological bouts of sniffing (Wachowiak, 2011). Glomerular activation thus acts to reset the phase of spontaneous firing in GL-dSACs. The high precision and temporal nature of this response to glomerular activation, together with highly regular theta-frequency spontaneous firing, suggests that GL-dSACs may be involved in the temporal patterning of specific aspects of MOB activity at theta frequencies (see Discussion).

Feedforward excitatory input to GL-dSACs may originate from ETCs, TCs, and/or MCs. However, at least three lines of evidence suggest that ETCs provide the primary feedforward excitatory input to GL-dSACs. First, the somata and dendrites of GL-dSACs overlap with ETC axons and TC axon collaterals extensively within the IPL/sGCL (Macrides et al., 1985; Liu and Shipley, 1994). This overlap provides an anatomical basis for ETC/TC-mediated input to GL-dSACs while strongly arguing against input from MC axon collaterals, which extend throughout the deep GCL (Macrides et al., 1985). Second, the short latency (Fig. 6H) and duration (Fig. 6I) of feedforward excitatory input to GL-dSACs markedly precedes the first-spike latency of MCs under identical experimental conditions (Burton and Urban, 2014, 2015). Third, the short duration (Fig. 6I) and burstlike nature (Fig. $6 E-G, R-U$ ) of feedforward excitatory input to GL-dSACs mirrors the ETC-mediated excitatory input to type 2 periglomerular cells (PGCs) (Hayar et al., 2004; Shao et al., 2009; $\mathrm{Najac}$ et al., 2015), but contrasts strongly with the long-lasting $\mathrm{M} / \mathrm{TC}$-mediated excitation observed in granule cells (GCs) (Burton and Urban, 2015; Geramita et al., 2016).

To further evaluate the source of feedforward excitatory input to GL-dSACs, we performed paired recordings of GL-dSACs and nearby MCs (Fig. 9A-D) while stimulating OSNs at intensities perithreshold for generating an all-or-none long-lasting depolarization (LLD) in the MC (Carlson et al., 2000; Gire and Schoppa, 2009). The MC LLD is mediated by the regenerative activation of all recurrently coupled ETCs within a glomerulus (De Saint Jan et al., 2009; Gire and Schoppa, 2009). If GL-dSACs receive excitatory input from MCs, then LLD generation should be an important predictor of excitatory input to GL-dSACs. Strikingly, however, GL-dSACs received robust excitatory input on both LLD success and LLD failure trials (Fig. 9E,F). Together with the three lines of evidence described above, this result strongly argues that ETCs (and possibly TCs) provide the primary feedforward excitatory input to GL-dSACs, and moreover that GL-dSACs are excited even when OSN input to the MOB fails to activate principal MCs.

ETC axons can extend long distances within the IPL (see Discussion) (Macrides et al., 1985; Liu and Shipley, 1994; Belluscio et al., 2002; Lodovichi et al., 2003). It is thus possible that GL-dSACs integrate ETC-mediated excitatory input from both nearby and distant glomeruli. To examine the convergence of feedforward excitatory input to GL-dSACs, we activated single glomeruli hundreds of micrometers away while recording excitatory input to GL-dSACs (Fig. 9A). In paired recordings from GL-dSACs and nearby MCs, activation of distant glomeruli (Fig. 9B) evoked no input to MCs (Fig. 9E), confirming that extracellular stimulation does not activate OSN axons in passage. In contrast to MCs, GL-dSACs received robust excitatory input (Fig. 9E), even when activating single glomeruli $>700 \mu \mathrm{m}$ anterior to the recorded cell (Fig. 9G-I). Equivalent results were likewise observed when activating single glomeruli posterior to the recorded cell (Fig. $9 \mathrm{~J}, \mathrm{~K})$. Collectively, our results thus show that GL-dSACs integrate highly convergent excitatory input from several glomeruli, suggesting broad odor tuning in vivo.

\section{Centrifugal cholinergic fibers innervate GL-dSACs}

Cholinergic signaling critically regulates olfaction (Devore and Linster, 2012; D'Souza and Vijayaraghavan, 2014; Linster and Fontanini, 2014; Linster and Cleland, 2016). Understanding how cholinergic signaling influences sensory processing in the MOB 

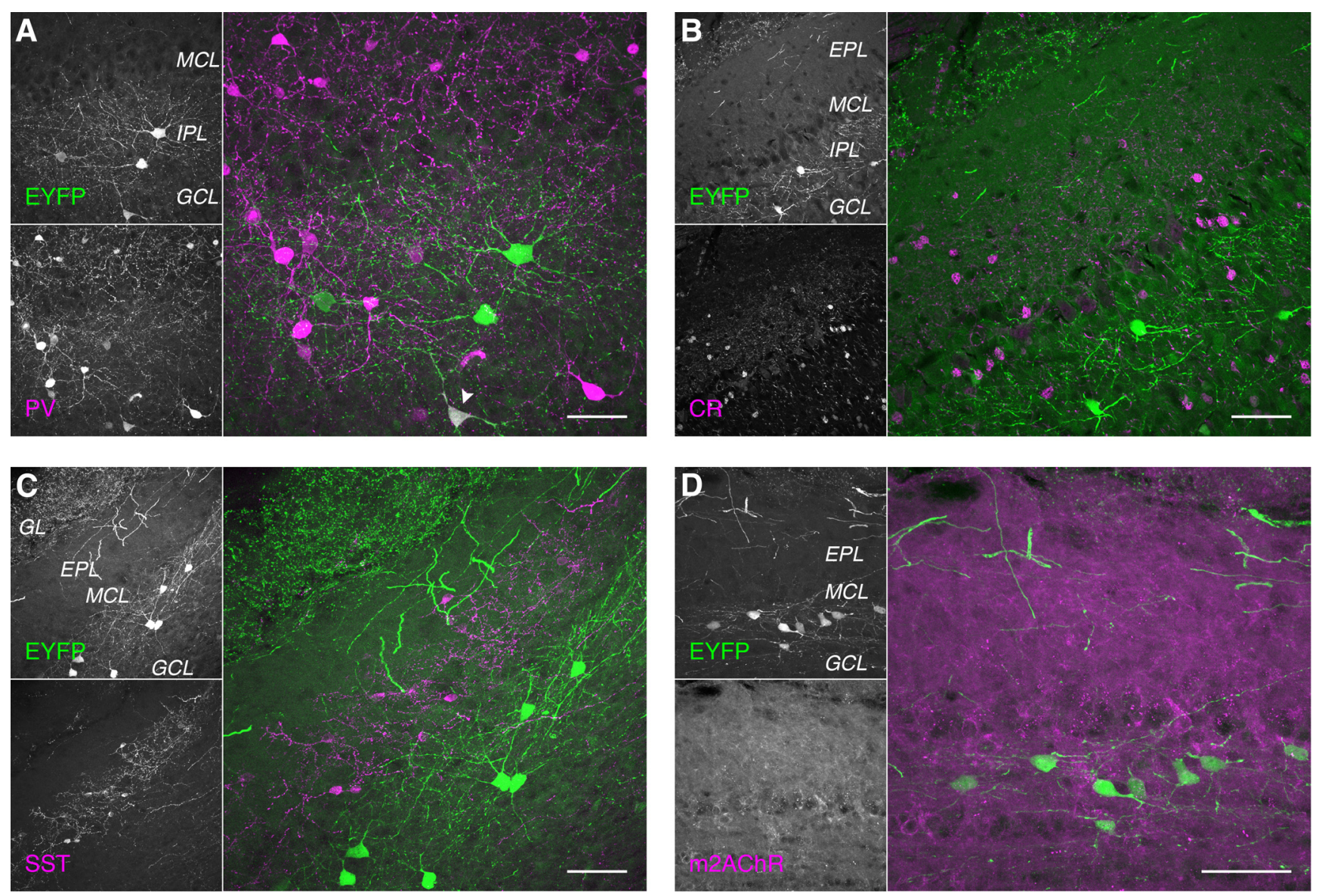

Figure 4. Neurochemical properties of GL-dSACs. A, Small subset of Chrna2-Cre-labeled GL-dSACs $(9.3 \% ; 4 / 43$ cells, $n=2$ mice) express parvalbumin (PV). Arrowhead marks a PV-expressing cell. B, C, Chrna2-Cre-labeled GL-dSACs do not express calretinin (CR) (B; 0.0\%; 0/25 cells, $n=2$ mice) or somatostatin (SST) (C; 0.0\%; 0/61 cells, $n=3$ mice). D, Chrna2-Cre-labeled dSACs exhibit negligible muscarinic AChR M2 (m2AChR) expression.

has proven difficult, however, because multiple neuron types express cholinergic receptors but exhibit no or weak response to endogenous ACh release (Liu et al., 2015; Smith et al., 2015). Expression of chrna2 suggests that GL-dSACs may express nAChRs and respond to endogenous ACh release, consistent with the dense cholinergic innervation of the IPL (Macrides et al., 1981; Shipley and Adamek, 1984).

We used a pharmacological approach to test for functional $\mathrm{nAChR}$ expression in GL-dSACs. Focal application of nicotinic agonist dimethylphenylpiperazinium (DMPP; $100 \mu \mathrm{M})$ (Fig. 10A) increased the spontaneous firing rate of GL-dSACs dramatically (Fig. $10 B-D, F)$. This increase in rate was driven by a non-desensitizing inward current, which was abolished by nicotinic antagonist mecamylamine (Mec; $20 \mu \mathrm{M})$ (Fig. 10E, G). Importantly, blockade of glutamatergic and GABAergic transmission had no effect on the response to DMPP (Fig. 10F, G), confirming that DMPP acts directly on functional nAChRs expressed by GL-dSACs.

To test whether GL-dSACs respond to endogenous ACh release, we recorded postsynaptic currents in GL-dSACs while stimulating the nearby IPL in the presence of glutamatergic and GABAergic antagonists (Fig. 10H). IPL stimulation evoked robust EPSCs in all GL-dSACs tested (Fig. 10I-L). These EPSCs were abolished by brief application of Mec (Fig. 10K) and exhibited kinetics consistent with the expression of $\alpha 2 \beta 4$-containing nAChRs (Fig. 10L) (Figl and Cohen, 2000). Of great interest, the observed nicotinic EPSCs are, to our knowledge, the largest response to endogenous $\mathrm{ACh}$ release observed among any $\mathrm{MOB}$ neuron type, implicating GL-dSACs as an important component of the cholinergic regulation of olfaction.

\section{GL-dSACs innervate PGCs}

To examine the synaptic output of GL-dSACs, we injected AAV into the $\mathrm{MOB}$ to drive Cre-dependent channelrhodopsin (ChR2):mCherry expression in GL-dSACs (Fig. 11A; see Materials and Methods). Photostimulation with brief (10 ms) blue light pulses reliably evoked one to four action potentials in GL-dSACs in the presence of glutamatergic antagonists (Fig. $11 B-H$ ), confirming the direct influence of ChR2 on GL-dSACs.

Previous ultrastructural examination of biocytin-filled GLdSAC axon terminals identified synaptic contacts onto putative PGCs (Eyre et al., 2008). Consistent with this result, a large subset of PGCs, which were identified by their small somata and dendritic tuft (Fig. 11I,J), exhibited robust, short-latency postsynaptic currents after brief ( $10 \mathrm{~ms}$ ) light pulses (Fig. $11 \mathrm{~K}-\mathrm{M}$ ). These postsynaptic currents were unaffected by glutamatergic antagonists but were abolished by subsequent application of GBZ (Fig. $11 K-N)$, consistent with monosynaptic release of GABA from GL-dSACs onto PGCs. Interestingly, the postsynaptic currents in PGCs reversed at a relatively depolarized potential ( $-44 \mathrm{mV}$; Fig. $11 N$ ) despite the recording electrode containing a low- $\mathrm{Cl}^{-}$internal solution (see Materials and Methods). This depolarized reversal potential is consistent with multiple previous reports (Smith and Jahr, 2002; Parsa et al., 2015), however, and suggests that GABA release from GL-dSACs may act to increase PGC firing, as 

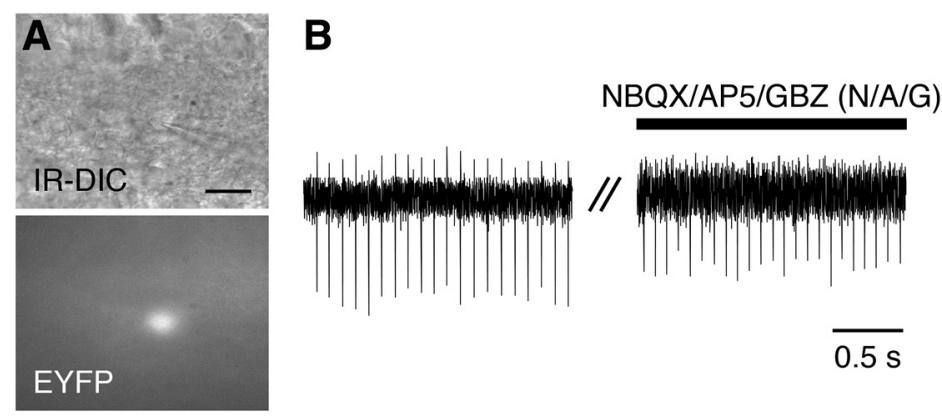

$\overline{0.5 \mathrm{~s}}$
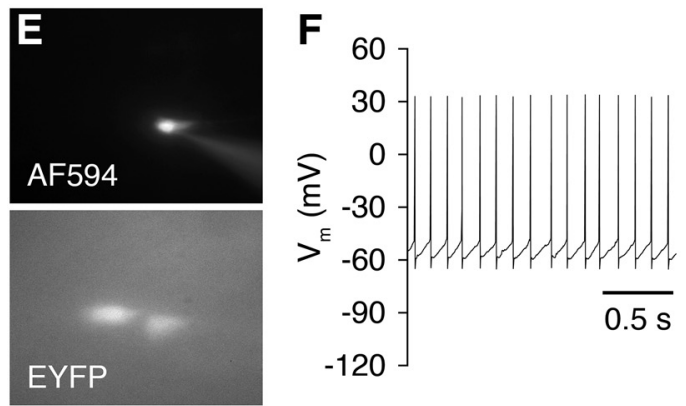

G
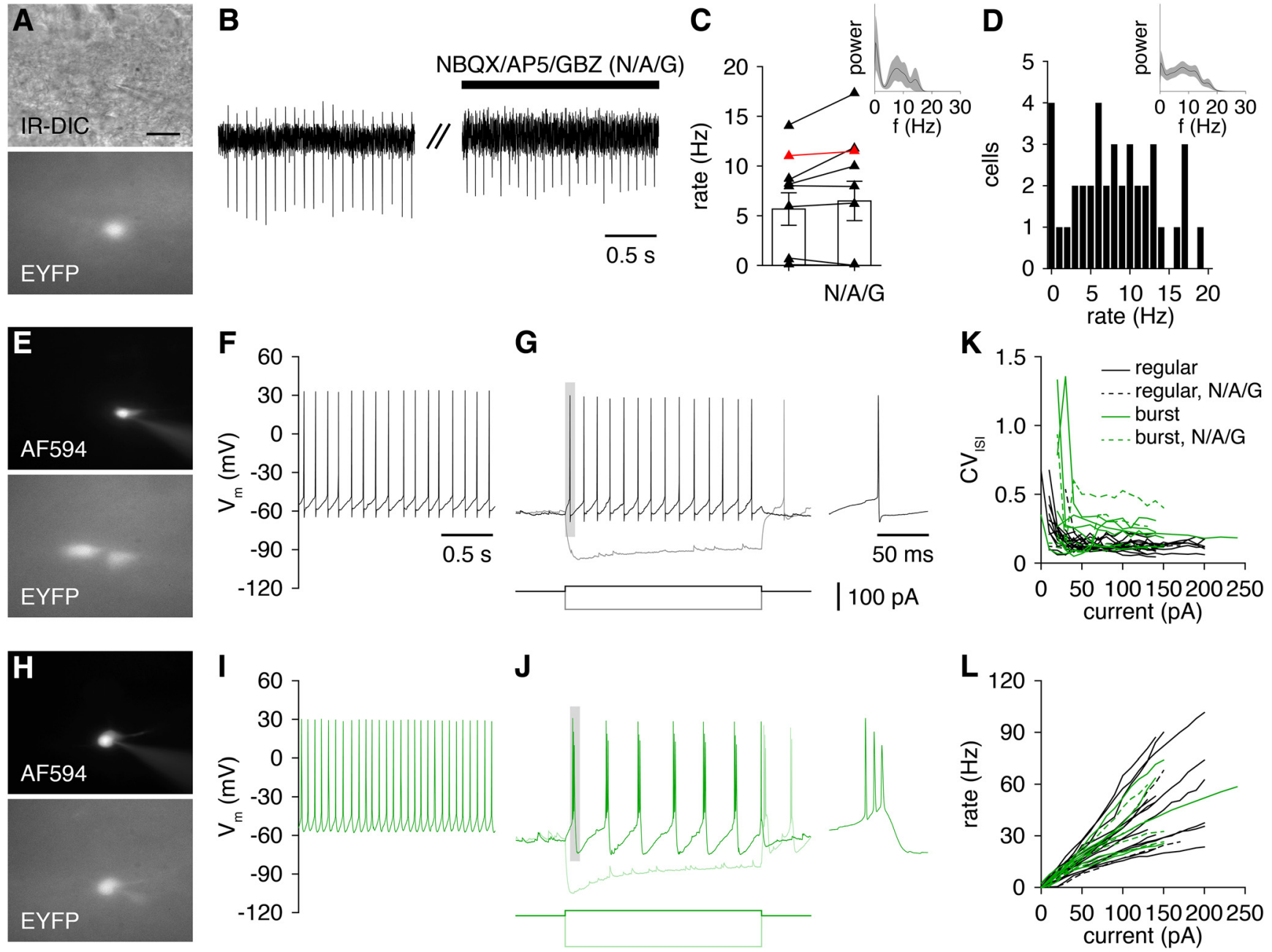
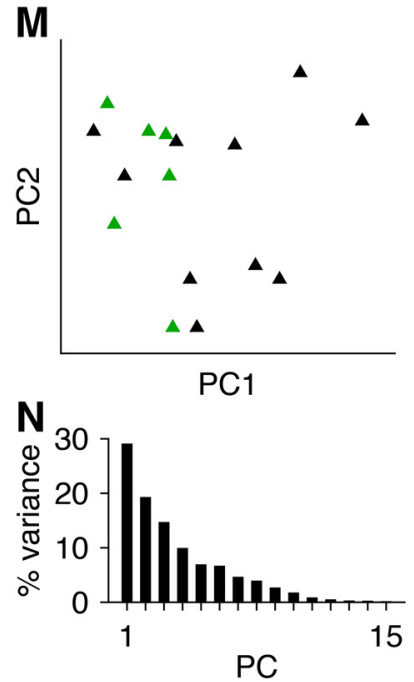
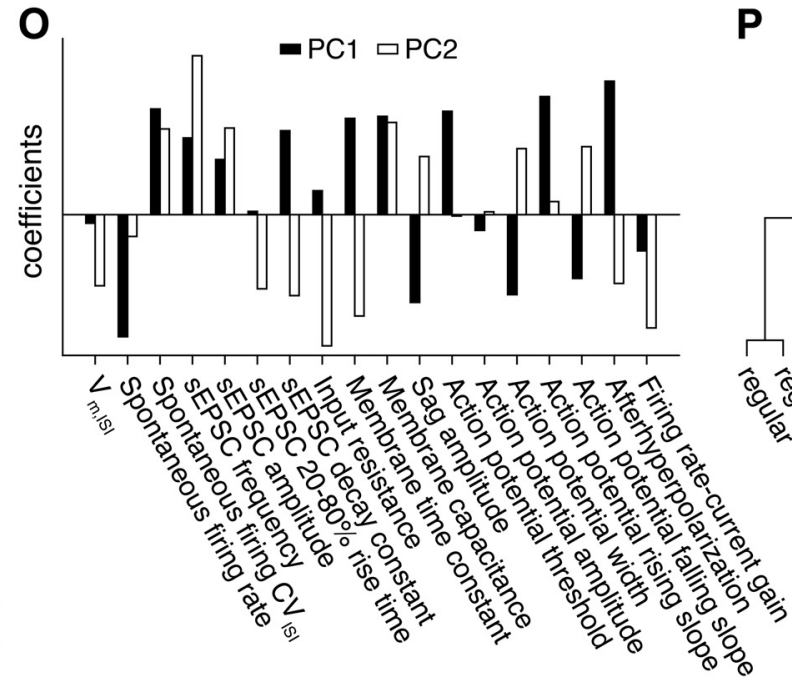

P

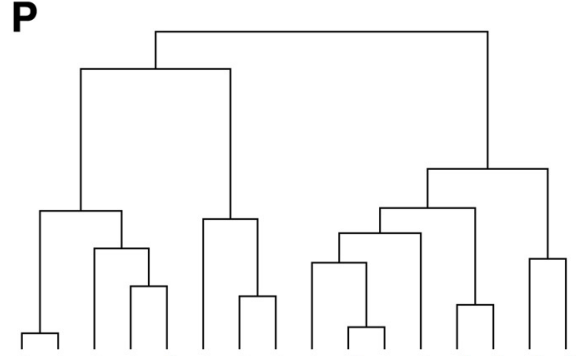

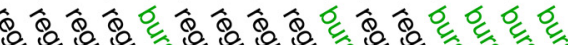

//, g/, //,

Figure 5. Functional properties of GL-dSACs. $A-C$, EYFP-expressing GL-dSACs fire tonically in cell-attached recordings independently of synaptic activity $(5.7 \pm 5.2 \mathrm{vs} 6.5 \pm 6.3 \mathrm{~Hz}$, before vs after NBQX/AP5/GBZ application; $n=10 ; p=0.10$, two-tailed paired $t$ test). Red points denote cell shown in $\boldsymbol{A}$ and $\boldsymbol{B}$. Inset, Mean power of cell-attached spike times ( $n=10$ ). $\boldsymbol{D}$, Distribution of GL-dSAC spontaneous firing rates recorded in whole-cell mode $(8.3 \pm 5.6 \mathrm{~Hz} ; n=33)$. Inset: mean power of spike times $(n=33)$. $\boldsymbol{E}-\mathbf{G}$, Whole-cell recordings from a representative $\mathrm{GL}$-dSAC showing regular firing at rest $(\boldsymbol{F})$ and at a hyperpolarized $V_{m}$ in response to a depolarizing step current (G). Single action potential expanded at right. $\boldsymbol{H}-\boldsymbol{J}$, Example GL-dSAC exhibiting burst firing when depolarized or hyperpolarized from a hyperpolarized $V_{m}$. Single burst is expanded at right. $\boldsymbol{K}, \mathrm{GL}-\mathrm{dSACs}$ exhibit highly regular firing in response to strong ( $>50 \mathrm{pA}$ ) depolarizing step currents (without $\mathrm{NBQX} / \mathrm{AP5} / \mathrm{GBZ}, n=20$; with NBQX/AP5/GBZ, $n=6$ ). $L$, GL-dSACs exhibit relatively uniform excitability in response to step current injections (without NBQX/AP5/GBZ, $n=20 ;$ with NBQX/AP5/GBZ, $n=6) . M$, Projection of regular firing (black) and bursting (green) GL-dSACs onto the first two principal components (PC1 and PC2) identified from principal component analysis of GL-dSAC functional properties. No obvious clustering of GL-dSACs by firing pattern emerged. $\boldsymbol{N}$, Variance in GL-dSAC functional properties explained by each principal component. $\mathbf{0}$, Decomposition of PC1 and PC2 into their constituent property coefficients. $\boldsymbol{P}$, Unbiased agglomerative hierarchical clustering of GL-dSACs by the Euclidean distances among the $z$-scored functional properties listed in $\mathbf{0}$ (using Ward's method). Application of the gap statistic method yielded one cluster. 


\begin{tabular}{|c|c|c|}
\hline & & $+\mathrm{NBQX/AP5/GBZ}$ \\
\hline \multicolumn{3}{|l|}{ Cell-attached recordings } \\
\hline Spontaneous firing rate $(\mathrm{Hz})$ & $5.7 \pm 5.2(0.24-8.5)(10)$ & $6.5 \pm 6.3(0.0-11.1)(10)$ \\
\hline Spontaneous firing $\mathrm{CV}_{\mathrm{ISI}}$ & $0.46 \pm 0.45(0.13-0.70)(10)$ & $0.21 \pm 0.22(0.08-0.20)(10)$ \\
\hline \multicolumn{3}{|l|}{ Whole-cell recordings } \\
\hline Spontaneous firing rate $(\mathrm{Hz})$ & $8.3 \pm 5.6(3.9-12.2)(33)$ & $8.2 \pm 4.1(6.3-11.5)(6)$ \\
\hline Spontaneous firing $\mathrm{CV}_{\mathrm{ISI}}$ & $0.19 \pm 0.21(0.08-0.21)(32)$ & $0.09 \pm 0.07(0.04-0.11)(6)$ \\
\hline$V_{\mathrm{m}, \mid \mathrm{SI}}(\mathrm{mV})$ & $-54.5 \pm 2.9(-56.0$ to -52.3$)(33)$ & $-54.4 \pm 4.4(-57.2$ to -51.1$)(6)$ \\
\hline \multicolumn{3}{|l|}{ sEPSC properties (Cs-internal) } \\
\hline Frequency $(\mathrm{Hz})$ & $10.0 \pm 5.4(6.3-12.1)(16)$ & - \\
\hline Amplitude (pA) & $32.2 \pm 8.2(27.1-34.5)(16)$ & - \\
\hline $20-80 \%$ rise time (ms) & $0.42 \pm 0.05(0.39-0.47)(16)$ & - \\
\hline Decay constant (ms) & $2.8 \pm 1.0(2.3-3.0)(16)$ & - \\
\hline \multicolumn{3}{|l|}{ sIPSC properties (Cs-internal) } \\
\hline Frequency $(\mathrm{Hz})$ & $12.8 \pm 5.2(8.7-16.9)(16)$ & - \\
\hline Amplitude (pA) & $38.6 \pm 8.0(32.4-45.4)(16)$ & - \\
\hline $20-80 \%$ rise time (ms) & $0.82 \pm 0.11(0.74-0.90)(16)$ & - \\
\hline Decay constant (ms) & $7.7 \pm 1.3(6.9-8.1)(16)$ & - \\
\hline Input resistance $(\mathrm{M} \Omega$ ) & $551.6 \pm 193.8(410.4-702.5)(18)$ & $429.0 \pm 110.8(355.4-478.3)(6)$ \\
\hline Membrane time constant (ms) & $25.8 \pm 7.2(21.4-30.6)(18)$ & $22.0 \pm 5.0(18.6-23.8)(6)$ \\
\hline Membrane capacitance (pF) & $48.6 \pm 10.2(43.1-54.4)(18)$ & $52.0 \pm 7.8(47.1-55.2)(6)$ \\
\hline Sag amplitude (mV) & $10.0 \pm 5.5(5.9-12.9)(17)$ & $7.7 \pm 6.4(4.4-9.7)(6)$ \\
\hline \multicolumn{3}{|l|}{ Action potential properties } \\
\hline Threshold (mV) & $-45.0 \pm 4.0(-48.1$ to -42.8$)(20)$ & $-43.6 \pm 7.8(-49.1$ to -37.5$)(6)$ \\
\hline Amplitude (mV) & $73.6 \pm 5.3(71.7-78.2)(20)$ & $71.2 \pm 2.7(69.7-72.5)(6)$ \\
\hline Width (ms) & $0.67 \pm 0.14(0.60-0.80)(20)$ & $0.60 \pm 0.09(0.53-0.68)(6)$ \\
\hline Rising slope (mV/ms) & $253.0 \pm 41.8(218.3-277.7)(20)$ & $247.2 \pm 38.6(236.5-260.8)(6)$ \\
\hline Falling slope (mV/ms) & $-121.2 \pm 31.0(-144.3$ to -98.0$)(20)$ & $-132.2 \pm 19.0(-141.3$ to -123.8$)(6)$ \\
\hline Afterhyperpolarization (mV) & $17.5 \pm 6.1(13.2-21.8)(20)$ & $15.3 \pm 5.0(11.2-19.2)(6)$ \\
\hline Firing rate current gain (Hz/pA) & $0.59 \pm 0.27(0.40-0.68)(20)$ & $0.53 \pm 0.16(0.46-0.61)(6)$ \\
\hline
\end{tabular}

Values are shown as mean $\pm \mathrm{SD}\left(Q_{1}-Q_{3}\right)(n)$.

Table 3. Functional differences between regular firing and bursting GL-dSACs

\begin{tabular}{|c|c|c|}
\hline & Regular firing & Bursting \\
\hline Spontaneous firing rate $(\mathrm{Hz})$ & $6.2 \pm 4.9(2.9-8.3)(14)$ & $10.6 \pm 6.0(8.5-12.9)(6)$ \\
\hline Spontaneous firing $\mathrm{CV}_{\mathrm{ISI}}$ & $0.24 \pm 0.19(0.13-0.27)(13)$ & $0.07 \pm 0.04(0.06-0.09)(6)^{\#}$ \\
\hline \multicolumn{2}{|l|}{ sEPSC properties (K-internal) } & $-53.9 \pm 3.2(-57.5$ to -51.4$)(6)$ \\
\hline Frequency $(\mathrm{Hz})$ & $11.9 \pm 9.8(4.8-20.6)(14)$ & $5.8 \pm 4.6(2.7-10.7)(6)$ \\
\hline Amplitude (pA) & $32.5 \pm 7.0(25.9-40.1)(14)$ & $30.5 \pm 5.0(26.0-34.8)(6)$ \\
\hline $20-80 \%$ rise time (ms) & $0.46 \pm 0.04(0.42-0.50)(14)$ & $0.42 \pm 0.05(0.40-0.42)(6)$ \\
\hline Decay constant (ms) & $2.2 \pm 0.4(2.0-2.5)(14)$ & $1.9 \pm 0.2(1.7-2.0)(6)$ \\
\hline Input resistance $(\mathrm{M} \Omega)$ & $605.6 \pm 163.5(441.9-747.3)(12)$ & $443.7 \pm 219.0(324.5-411.8)(6)$ \\
\hline Membrane time constant (ms) & $28.3 \pm 6.0(23.9-30.9)(12)$ & $20.7 \pm 7.1(16.6-24.8)(6)$ \\
\hline Membrane capacitance (pf) & $48.5 \pm 10.7(43.2-52.2)(12)$ & $48.8 \pm 10.0(38.8-57.6)(6)$ \\
\hline Sag amplitude (mV) & $8.5 \pm 4.9(4.6-11.1)(11)$ & $12.7 \pm 5.8(6.3-16.0)(6)$ \\
\hline \multicolumn{3}{|l|}{ Action potential properties } \\
\hline Threshold (mV) & $-44.9 \pm 4.3(-48.8$ to -43.8$)(14)$ & $-45.3 \pm 3.6(-47.8$ to -42.7$)(6)$ \\
\hline Amplitude (mV) & $73.2 \pm 5.3(71.5-78.1)(14)$ & $74.6 \pm 5.8(73.2-79.0)(6)$ \\
\hline Width (ms) & $0.68 \pm 0.16(0.60-0.80)(14)$ & $0.65 \pm 0.08(0.60-0.70)(6)$ \\
\hline Rising slope (mV/ms) & $253.9 \pm 48.4(214.4-282.8)(14)$ & $251.0 \pm 23.1(230.0-270.0)(6)$ \\
\hline Falling slope (mV/ms) & $-121.9 \pm 32.9(-152.5$ to -97.8$)(14)$ & $-119.8 \pm 29.1(-131.9$ to -98.8$)(6)$ \\
\hline Afterhyperpolarization (mV) & $20.1 \pm 4.6(17.8-24)(14)$ & $11.3 \pm 4.2(9.4-13.7)(6)^{*}$ \\
\hline Firing rate current gain $(\mathrm{Hz} / \mathrm{pA})$ & $0.55 \pm 0.28(0.35-0.65)(14)$ & $0.67 \pm 0.25(0.48-0.90)(6)$ \\
\hline
\end{tabular}

${ }^{*} p<0.05$, two-tailed unpaired $t$ test with Bonferroni correction for multiple comparisons; $\# p<0.05$, two-sided Wilcoxon rank-sum test with Bonferroni correction for multiple comparisons. Values are shown as mean $\pm S D$ $\left(Q_{1}-Q_{3}\right)(n)$.

demonstrated recently with exogenous GABA application (Parsa et al., 2015).

Approximately one-third of PGCs (i.e., type 1 PGCs) receive direct sensory-evoked input from OSNs and colocalize with OMP-positive glomerular compartments, whereas the remaining PGCs (i.e., type 2 PGCs) colocalize with OMPnegative glomerular compartments and receive indirect feedforward sensory-evoked input from ETCs (Hayar et al., 2004;
Shao et al., 2009; Nagayama et al., 2014; Najac et al., 2015). To investigate whether GL-dSACs target type 1 and/or 2 PGCs, we first examined the overlap between GL-dSAC axonal boutons and OMP-positive glomerular compartments. EYFPexpressing GL-dSAC boutons innervated OMP-negative compartments profusely while strikingly avoiding OMP-positive compartments (Fig. 12A), strongly arguing that GL-dSACs target type 2 PGCs. 
Table 4. Morphological differences between regular firing and bursting GL-dSACs

\begin{tabular}{|c|c|c|}
\hline & Regular firing & Bursting \\
\hline \multicolumn{3}{|l|}{ Soma } \\
\hline $\operatorname{Area}\left(\mu \mathrm{m}^{2}\right)$ & $254.9 \pm 52.7(232.0-258.16)(6)$ & $196.2 \pm 39.2(168.6-223.9)(4)$ \\
\hline Major diameter ( $\mu \mathrm{m})$ & $22.4 \pm 3.4(20.0-24.7)(6)$ & $19.9 \pm 2.8(17.7-22.2)(4)$ \\
\hline Minor diameter ( $\mu \mathrm{m})$ & $15.7 \pm 1.2(14.8-16.9)(6)$ & $13.1 \pm 1.1(12.3-13.8)(4)$ \\
\hline \multicolumn{3}{|l|}{ Dendrite } \\
\hline Total length $(\mu \mathrm{m})$ & $985.8 \pm 208.0(832.4-1064.5)(6)$ & $1541.1 \pm 164.1(1401.7-1680.5)(4)^{*}$ \\
\hline Total volume $\left(\mu \mathrm{m}^{3}\right)$ & $624.3 \pm 160.4(499.1-761.3)(6)$ & $758.8 \pm 306.0(522.6-995.0)(4)$ \\
\hline Spines & $3.5 \pm 2.3(1.0-6.0)(6)$ & $8.3 \pm 4.5(5.0-11.5)(4)$ \\
\hline Branch points & $7.7 \pm 1.6(6.0-9.0)(6)$ & $14.0 \pm 2.2(12.5-15.5)(4)^{*}$ \\
\hline Length in MCL (\%) & $18.7 \pm 13.1(4.5-25.2)(6)$ & $8.3 \pm 3.3(6.2-10.4)(4)$ \\
\hline Length in IPL (\%) & $76.5 \pm 16.2(62.4-95.5)(6)$ & $60.5 \pm 23.8(45.7-75.4)(4)$ \\
\hline Length in GCL (\%) & $4.9 \pm 10.9(0.0-1.8)(6)$ & $31.2 \pm 23.8(15.0-47.4)(4)$ \\
\hline Maximum Sholl radius ( $\mu \mathrm{m})$ & $161.7 \pm 42.6(150.0-180.0)(6)$ & $187.5 \pm 59.1(150.0-225.0)(4)$ \\
\hline Fractal index & $1.06 \pm 0.03(1.03-1.08)(6)$ & $1.09 \pm 0.01(1.09-1.10)(4)$ \\
\hline \multicolumn{3}{|l|}{ Somatodendritic } \\
\hline Convex hull volume $\left(\times 10^{5} \mu \mathrm{m}^{3}\right)$ & $3.4 \pm 2.4(1.5-5.3)(6)$ & $4.1 \pm 2.3(2.9-5.4)(4)$ \\
\hline
\end{tabular}

${ }^{*} p<0.05$, two-tailed unpaired $t$ test with Bonferroni correction for multiple comparisons. Values are shown as mean $\pm S D\left(Q_{1}-Q_{3}\right)(n)$.

Type 2 PGCs can be subdivided functionally into nonoverlapping populations of calbindin- and calretinin-expressing PGCs (CB- and CR-PGCs, respectively) (Nagayama et al., 2014; Najac et al., 2015). Importantly, CB-PGCs are strongly connected to the glomerular microcircuit and exhibit large sEPSCs with frequencies $>8 \mathrm{~Hz}$, whereas CR-PGCs are weakly connected to the glomerular microcircuit and exhibit small sEPSCs with frequencies $<8 \mathrm{~Hz}$ (Najac et al., 2015). Subdividing PGCs by their sEPSC frequencies (Fig. $12 B-D, F-H$ ) revealed that GL-dSACs innervate both functional subtypes of type 2 PGCs (Fig. 12I,J). Moreover, GL-dSAC input to PGCs was highly reliable and showed only modest depression at theta frequencies (Fig. 12E). GLdSACs thus innervate both subtypes of type 2 PGCs to regulate inhibitory circuits in the GL.

\section{GL-dSACs innervate ETCs and TCs and can drive selective long-lasting inhibition of TCs}

We next investigated whether GL-dSAC photostimulation evoked postsynaptic currents in other MOB cell types within or connected to the GL, including ETCs, TCs, and MCs. Given previous ultrastructural data suggesting that GL-dSACs target GABAergic neurons exclusively (Eyre et al., 2008), we expected that GL-dSAC photostimulation might influence glutamatergic MOB neurons indirectly via polysynaptic pathways. Surprisingly, however, ETCs, which were identified by their large apical dendritic tuft and lack of lateral dendrites (Fig. 13A,F), exhibited reliable, short-latency postsynaptic currents in response to GLdSAC photostimulation (Fig. 13K), suggesting instead that ETCs receive direct GL-dSAC input. Indeed, the latency of input to ETCs was not significantly different from the latency of input to PGCs (Fig. 13T). Our results thus suggest that both PGCs and ETCs receive monosynaptic input from GL-dSACs. Compared with PGCs, however, the reversal potential of GL-dSAC input to ETCs was significantly more hyperpolarized (Fig. 11N), suggesting that GL-dSACs inhibit ETCs.

In striking contrast to the postsynaptic currents observed in PGCs and ETCs, brief GL-dSAC photostimulation evoked robust, short-latency, long-lasting inhibitory currents in 14 of 18 TCs, including four of eight superficial TCs (sTCs) located near the GL border (Fig. $13 B, G, L$ ) and 10 of 10 middle TCs (mTCs) (Fig. 13C, $H, M$ ). The four sTCs that failed to exhibit long-lasting inhibitory currents instead displayed synaptic responses similar to ETCs and thus likely correspond to the subset of ETCs that bear lateral dendrites (Nagayama et al., 2014). To confirm that these distinct synaptic responses were not artifacts arising from slice-to-slice or glomerulus-to-glomerulus differences, we additionally performed paired recordings of ETCs and TCs connected to the same glomerulus (Fig. 13D,I) and observed identical results (Fig. 13N). GL-dSAC photostimulation thus evokes highly distinct modes of inhibitory input to ETCs and TCs, the first such circuit that has been observed. We additionally confirmed that these postsynaptic responses were specific to GL-dSAC photostimulation and not due to retrograde infection of centrifugal inputs to the MOB (data not shown).

Surprisingly, MCs exhibited either no postsynaptic response to GL-dSAC photostimulation (Fig. $13 \mathrm{E}, J, O$ ) or a very weak long-lasting inhibitory current (Fig. 13Q-S). Moreover, synaptic latencies were equivalent among PGCs, ETCs, and TCs, but significantly longer in MCs (Fig. 13T). Our results therefore suggest that GL-dSACs target PGCs, ETCs, and TCs monosynaptically and also trigger a long-lasting inhibitory current in TCs that drives robust TC hyperpolarization (Fig. $13 U)$ and weak MC inhibition, potentially via GABA spillover. Although our results cannot exclude the potential contribution of glomerular gap junctions to the postsynaptic responses observed, monosynaptic GL-dSAC input to ETCs and TCs is consistent with the high density of presynaptic dSACs labeled in recent monosynaptic viral tracing of M/TCs (Miyamichi et al., 2013).

The long-lasting inhibitory currents observed in TCs exhibited several noteworthy features. First, these currents were remarkably consistent across trials and occurred in an all-or-none manner independent of the duration of GL-dSAC photostimulation (Fig. 14). Second, these currents markedly depressed at theta-frequency photostimulation (Fig. 13P), suggesting a strong refractoriness for the generation of the long-lasting inhibitory currents. Third, these currents were recorded using a $\mathrm{Cs}^{+}$-based internal solution (see Materials and Methods), thereby excluding $\mathrm{GABA}_{\mathrm{B}} \mathrm{R}$-mediated $\mathrm{K}^{+}$ channel modulation as a mechanism. Fourth, these currents occurred independent of glutamatergic transmission but were abolished by GBZ ( $n=3$; Fig. 13M). Fifth, these currents were absent from TCs with apical dendritic tufts truncated during slice preparation (Fig. 13U). Finally, these currents failed to reverse at negative holding potentials (Fig. $13 L-N$ ). In total, the combination of these features outlines a novel GABAergic signaling mechanism within the glomerulus (see Discussion). 
A

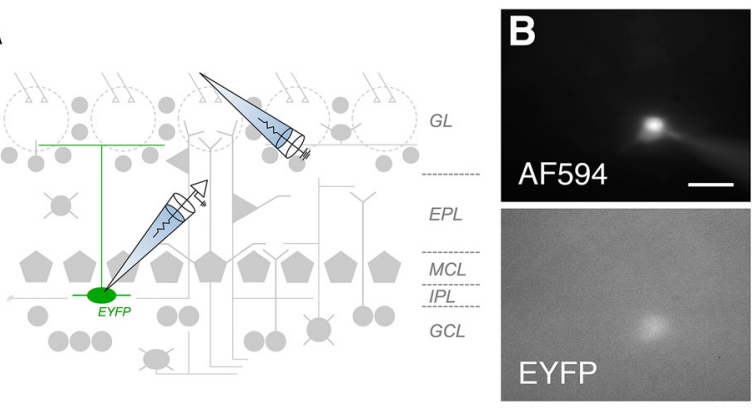

E
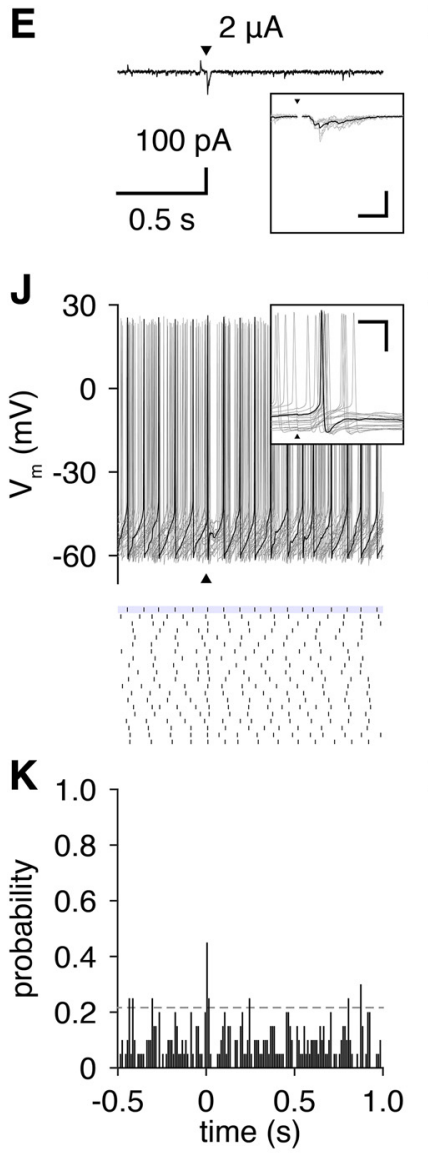

F

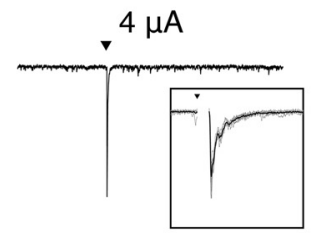

L

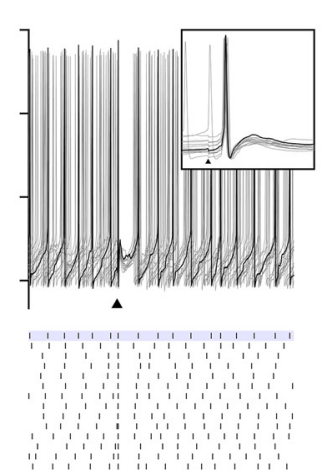

M

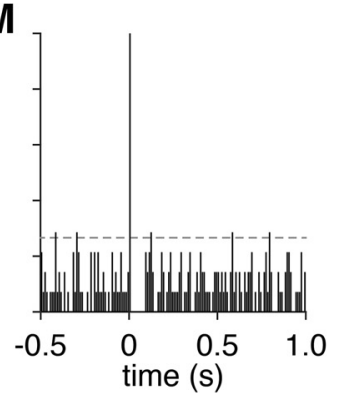

C

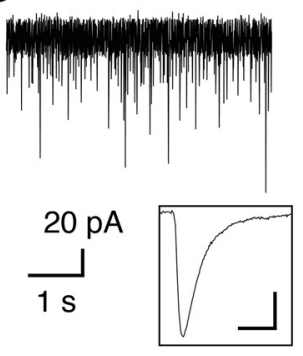

G

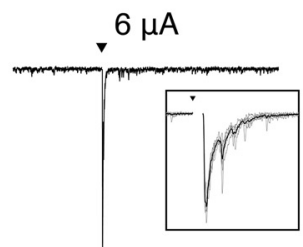

N

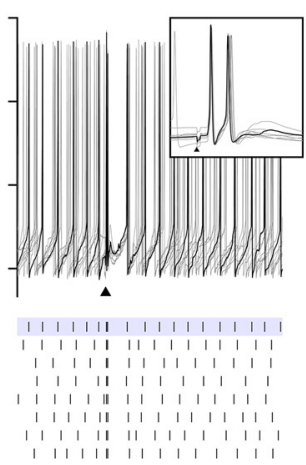

0

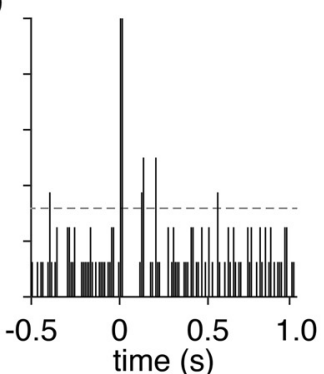

$\mathbf{U}$

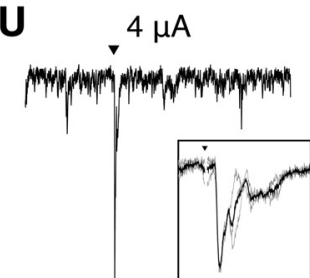

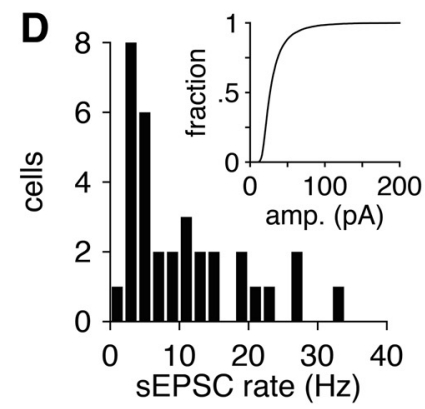
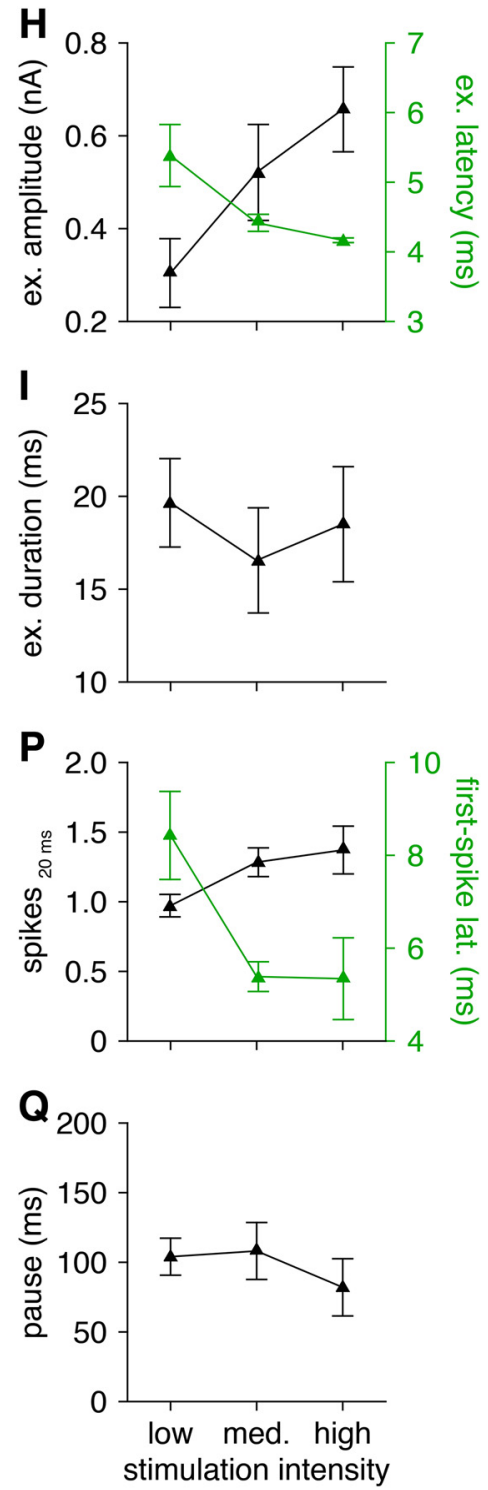

Figure 6. OSN stimulation resets the spontaneous firing phase of GL-dSACS. $A$, Excitatory input to $\mathrm{GL}-\mathrm{dSACS}$ was recorded after activation of a single glomerulus by $0 \mathrm{SN}$ stimulation. $\boldsymbol{B}$, Whole-cell recording of a representative EYFP-expressing GL-dSAC. Scale bar, $20 \mu \mathrm{m}$. C, Example recording of sEPSCs from the cell shown in $\boldsymbol{B}$. Inset, Mean sEPSC (scale bar, $4 \mathrm{~ms} / 10 \mathrm{pA})$. $\boldsymbol{D}$, Distribution of GL-dSAC sEPSC rates $(10.4 \pm 8.2 \mathrm{~Hz}, n=33)$. Inset, Distribution of sEPSC amplitudes. $\boldsymbol{E}$, Low-intensity OSN stimulation (arrowhead) evoked a short burst of EPSCs in the cell shown in $\boldsymbol{B}$. Mean response shown. Inset, Magnification of synaptic response (scale bar, $10 \mathrm{~ms} / 200 \mathrm{pA}$ ). Individual trials are shown in a lighter color. $\boldsymbol{F}, \mathbf{G}$, Same as $\boldsymbol{E}$ for medium-intensity $(\boldsymbol{F})$ and high-intensity $(\boldsymbol{G})$ OSN stimulation. $\boldsymbol{H}, \boldsymbol{I}$, Mean amplitude $(\boldsymbol{H})$, latency $(\boldsymbol{H})$, and duration $(\boldsymbol{I})$ of feedforward excitatory input to $\mathrm{GL}$-dSACs after low-intensity $(2.5 \pm 1.4 \mu \mathrm{A} ; n=12)$, medium-intensity $(4.3 \pm 2.3 \mu \mathrm{A}$; $n=6$ ), and high-intensity (5.3 $\pm 1.2 \mu \mathrm{A} ; n=3)$ OSN stimulation. J, Low-intensity OSN stimulation (arrowhead) evoked a single action potential and partially reset the spontaneous firing phase of the cell shown in $\boldsymbol{B}$. Multiple trials are shown (top), with a single trial plotted in black for clarity and a raster plot of spike times across trials (bottom; highlighted trial corresponds to trial plotted in black). Inset: magnification of spiking response (scale bar, $10 \mathrm{~ms} / 20 \mathrm{mV}$ ). $\boldsymbol{K}$, PSTH (10 ms bins) shows a rapid peak in action potential probability after 0SN stimulation at $0 \mathrm{~s}$ (dashed line: $95 \%$ confidence interval of basal action potential probability). $\boldsymbol{L}-\mathbf{O}$, Same as $\boldsymbol{J}$ and $\boldsymbol{K}$ for medium-intensity $(\boldsymbol{L}, \boldsymbol{M})$ and high-intensity $(\boldsymbol{N}, \mathbf{0})$ OSN stimulation. $\boldsymbol{P}$, Mean number of action potentials evoked within $20 \mathrm{~ms}$ after low-, medium-, and high-intensity OSN stimulation. Mean first-spike latency is plotted on the secondary axis. $\mathbf{Q}$, Mean pause in spontaneous firing following feedforward sensory-evoked firing. $\boldsymbol{R}-\boldsymbol{U}$, Two representative GL-dSACs with truncated axons (arrowheads) exhibiting excitatory input after OSN stimulation. Cell numbers and color code are reproduced from Figure 3. Morphology scale bars, $50 \mu \mathrm{m}$. Inset scale bar, $10 \mathrm{~ms} / 50 \mathrm{pA}$. 

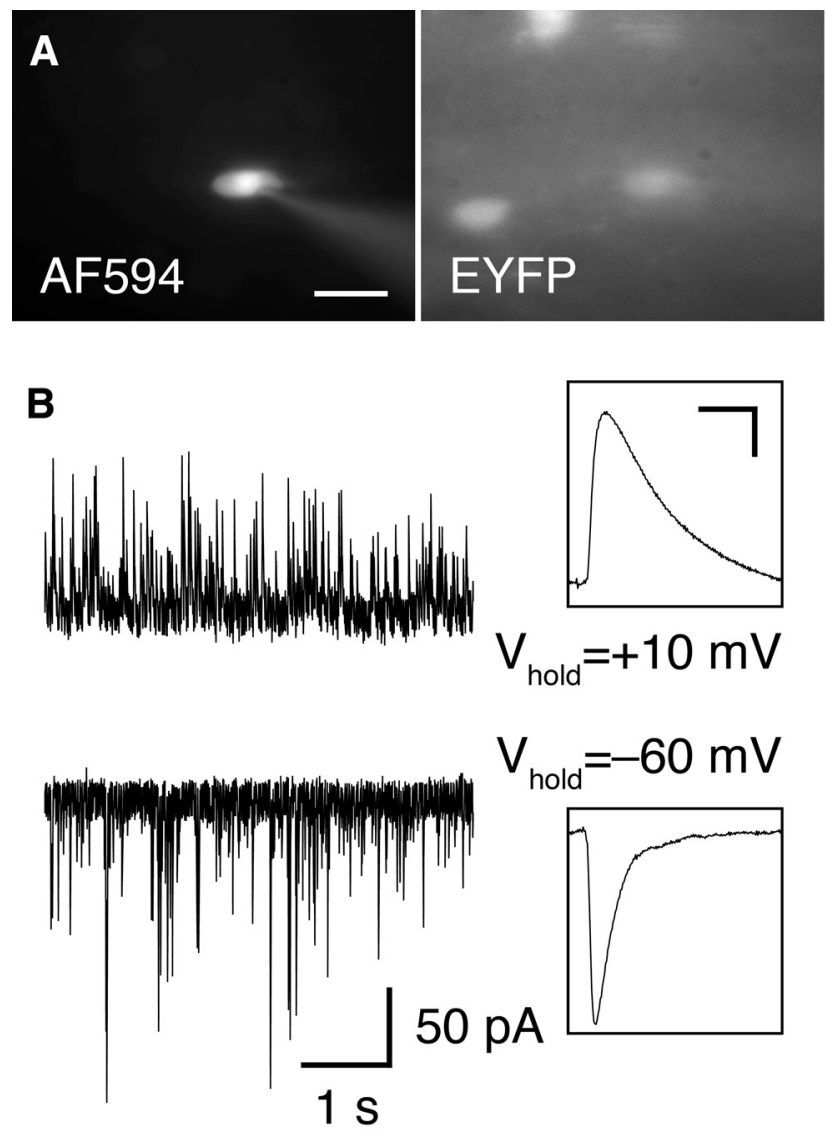
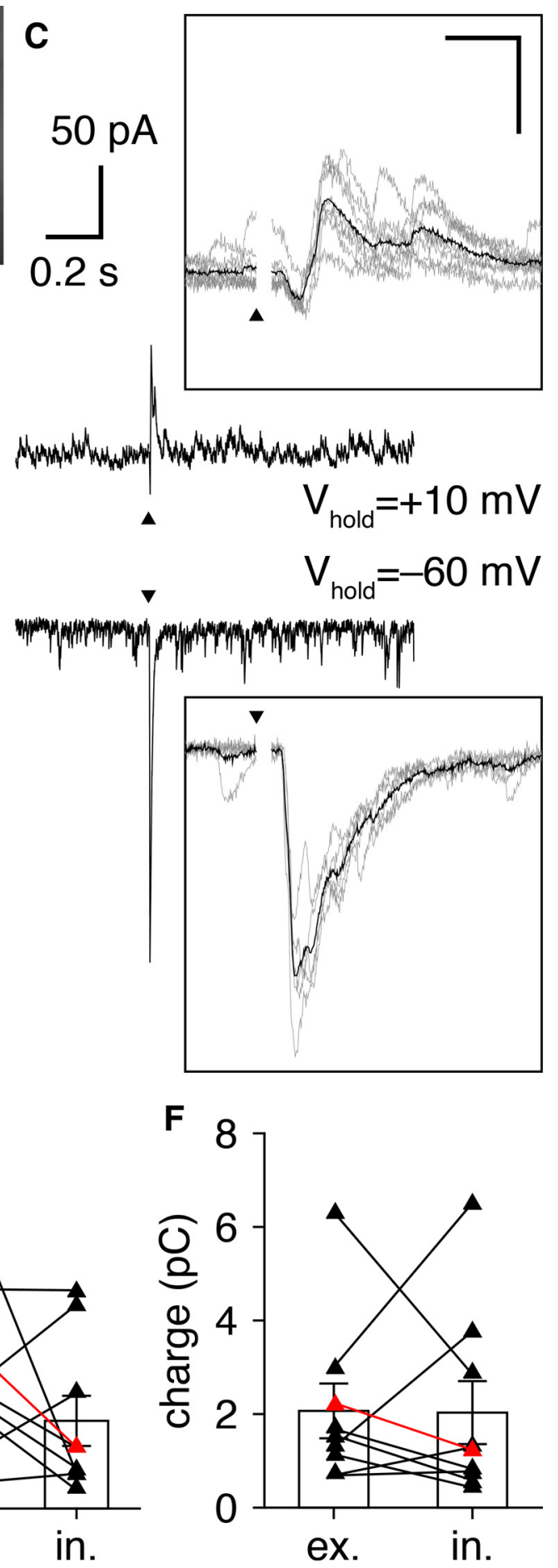

Figure 7. $\quad$ OSN stimulation evokes feedforward inhibition onto GL-dSACs. $\boldsymbol{A}$, Whole-cell recording of a representative EYFP-expressing GL-dSAC. Scale bar, $20 \mu \mathrm{m}$. $\boldsymbol{B}$, Example recording of sEPSCs and sIPSCs from the cell shown in $\boldsymbol{A}$. Inset, Magnification of mean sIPSC and sEPSC waveforms (scale bar, $5 \mathrm{~ms} / 10 \mathrm{pA}$ ). $\boldsymbol{C}, 0$ OSN stimulation (black triangle) evokes short-latency excitatory input, followed by a barrage of IPSCs in the cell shown in $\boldsymbol{A}$. Results are plotted as in Figure 6. Inset scale bar, $10 \mathrm{~ms} / 100 \mathrm{pA}$. $\boldsymbol{D}-\boldsymbol{F}$, Inhibitory input to GL-dSACs ( $\boldsymbol{n}=9$ ) exhibited longer latencies ( $\boldsymbol{D} ; 3.8 \pm$ 0.6 vs $7.9 \pm 3.6 \mathrm{~ms}$, excitatory vs inhibitory input; $p=3.9 \times 10^{-3}$, two-sided Wilcoxon signed-rank test), but comparable amplitude ( $\boldsymbol{E} ; 191.6 \pm 131.3$ vs $117.1 \pm 100.5$ pA, excitatory vs inhibitory input; $p=0.21$, two-tailed paired $t$ test) and charge transferred ( $\boldsymbol{F} ; 2.1 \pm 1.8 \mathrm{vs} 2.0 \pm 2.0 \mathrm{pC}$, excitatory vs inhibitory input; $p=0.73$, two-sided Wilcoxon signed-rank test) as excitatory input after OSN stimulation. Red points denote cell shown in $\boldsymbol{A}$ and $\boldsymbol{B}$.

In our final experiment, we investigated how GL-dSACmediated inhibition influences sensory-evoked TC activity. Specifically, we photostimulated GL-dSACs before, during, and after the activation of individual glomeruli via extracellular OSN stimulation. GL-dSAC photostimulation before or during OSN stimulation did not affect TC firing significantly (Fig. $13 \mathrm{~V}, \mathrm{~W}$ ).
However, GL-dSAC photostimulation tens of milliseconds after OSN stimulation, times matching differences in glomerular activation latencies observed in vivo (Wachowiak, 2011), truncated all late TC firing robustly (Fig. $13 \mathrm{~V}, W$ ). Synchronous GL-dSAC activation may thus be involved in regulating the sniff phase coupling of TC activity in vivo, facilitating the temporal coding of 

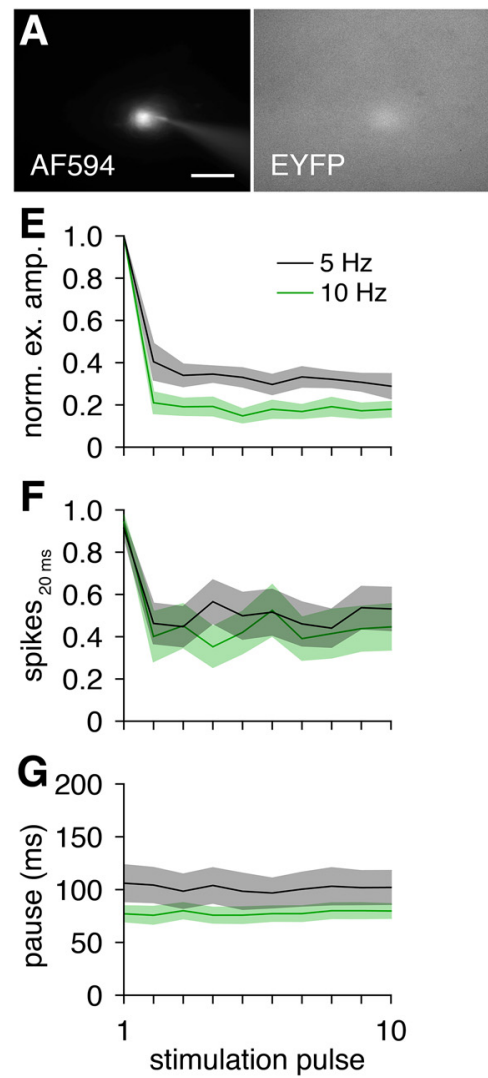

B

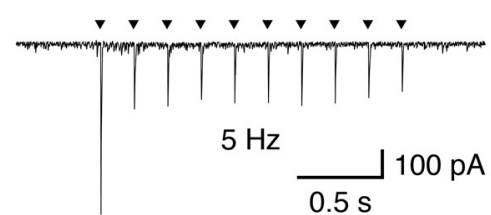

C

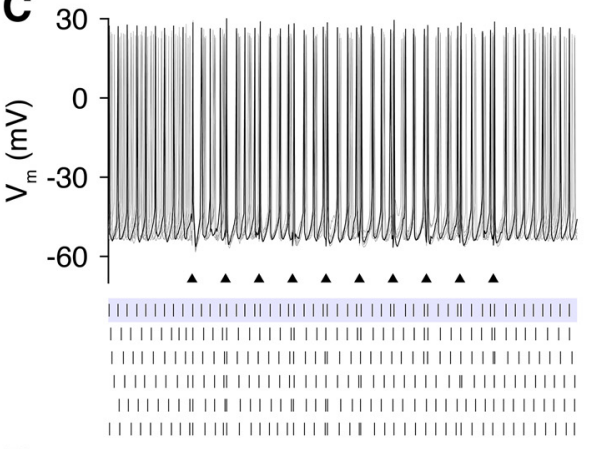

D

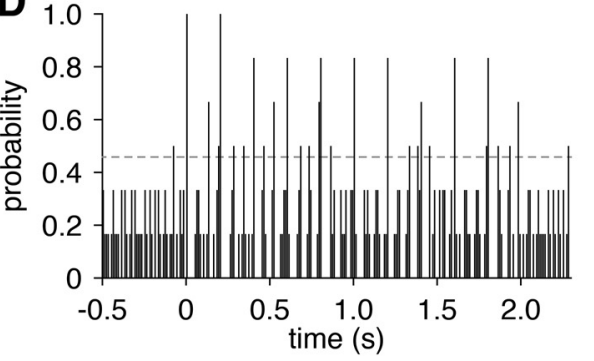

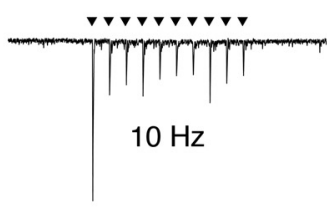
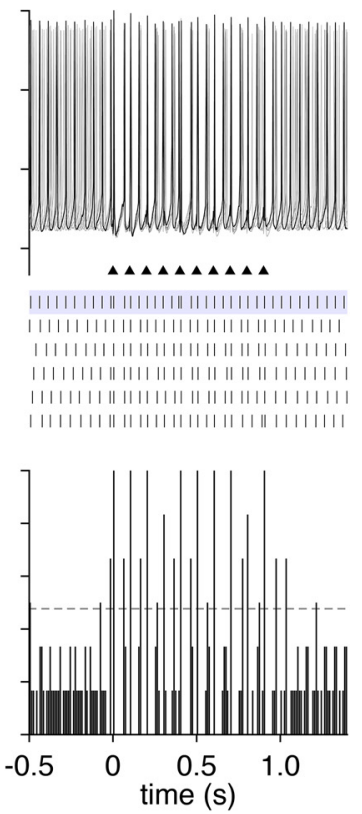

Figure 8. GL-dSAC firing tracks theta-frequency OSN stimulation reliably. A, Whole-cell recording of a representative EYFP-expressing GL-dSAC. Scale bar, $20 \mu \mathrm{m}$. B, Train of 0SN stimuli (black triangles) at 5 or $10 \mathrm{~Hz}$ evoked a time-locked train of depressing excitatory input to the cell shown in $\boldsymbol{A}$. C, $\boldsymbol{D}$, Train of OSN stimuli (black triangles) at 5 or $10 \mathrm{~Hz}$ evoked single time-locked action potentials in the cell shown in $A$. Results are plotted as in Figure 6 . $E$, Mean amplitude of feedforward excitatory input to GL-dSACs across 10 oSN stimulation pulses at 5 ( $n=10)$ or $10 \mathrm{~Hz}(n=7)$ normalized to the first stimulation pulse response. Excitatory input to GL-dSACs exhibits substantial initial frequency-dependent depression, followed by stable responses. $F$, Mean number of action potentials evoked within $20 \mathrm{~ms}$ after each of 10 OSN stimulation pulses at $5(n=11)$ or $10 \mathrm{~Hz}(n=11)$. G, Mean pause in spontaneous firing after OSN stimulation-evoked firing in each of 10 0SN stimulation pulses at $5(n=11)$ or $10 \mathrm{~Hz}(n=11)$.

sensory information by parallel TC and MC channels (Fukunaga et al., 2012).

\section{Discussion \\ Overview}

Understanding how inhibition shapes sensory processing in the olfactory system has been limited by the lack of selective markers for distinct MOB interneurons. Here, we have identified chrna2 as the first selective marker of GL-dSACs and used Chrna2-Cre mice to identify several structural and functional features by which GLdSACs may critically regulate MOB sensory processing.

Structurally, GL-dSACs are prominently located in the IPL, where they are ideally positioned to integrate centrifugal cholinergic input with feedforward sensory input from the axons of ETCs (and possibly TCs) of several glomeruli. This highly convergent feedforward sensory input is paralleled by highly divergent GL-dSAC output, mediated by extensive GL-dSAC axonal arborization across multiple glomeruli. These structural features make GL-dSACs uniquely suited to regulate large expanses of the $\mathrm{MOB}$ circuit across distinct brain states and in response to diverse sensory input.

Functionally, GL-dSACs intrinsically fire at theta frequencies and reset their spontaneous firing phase in response to feedforward sensory input. GABA release from GL-dSACs mediates remarkably celltype-specific effects, including PGC inhibition and/or excitation, ETC inhibition, and long-lasting inhibition of TC apical dendrites. In turn, GL-dSACs do not target MCs directly, a striking omission of a large class of $\mathrm{MOB}$ principal neurons.
Importantly, these structural and functional properties reflect the intrinsic properties of GL-dSACs and the MOB circuit and are thus independent of the acute slice preparation used in this study. Moreover, whereas our physiological experiments used relatively young, $\sim 3$ week-old mice with a mixed genetic background (see Materials and Methods), we observed identical properties (including somatodendritic morphologies, theta-frequency spontaneous firing, prodigious sEPSC and sIPSC rates, and robust nicotinic responses to endogenous ACh release) across 7 morphologically confirmed GL-dSACs recorded from $\sim 5$ week-old mice (P35.4 \pm 4.2$)$ with a C57BL/6 background (data not shown). Our results thus identify novel features of the MOB circuit that are conserved across mouse strains and developmental stages.

Although the exact roles of GL-dSACs in olfaction remain to be examined in vivo, the findings presented here collectively suggest that GL-dSAC activity may be critically involved in temporally patterning aspects of $\mathrm{MOB}$ activity while also supporting parallel processing of sensory information by MCs and TCs. In particular, the unique input and output connectivity of GLdSACs suggests a possible role in shifting the balance of activity between MCs and TCs.

\section{Emergent interneuron features}

Our results suggest that PGCs, ETCs, and TCs all receive monosynaptic input from GL-dSACs. This finding putatively identifies GL-dSACs as the first class of MOB interneurons 
A

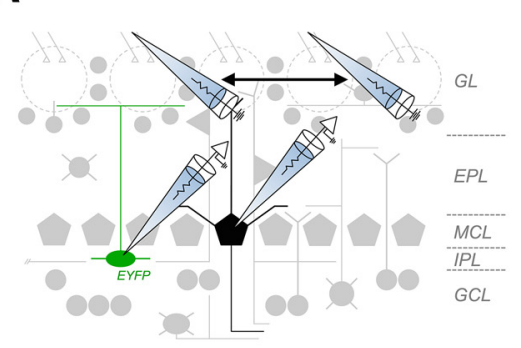

E

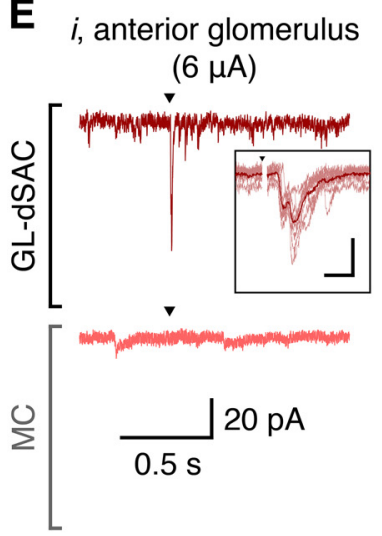

B

ii, proximal glomerulus (2 $\mu \mathrm{A}$; LLD failure)

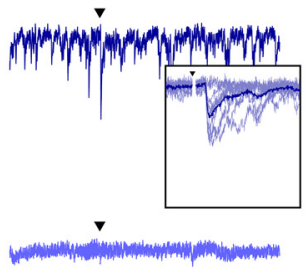

ii, proximal glomerulus (2 $\mu \mathrm{A}$; LLD success)

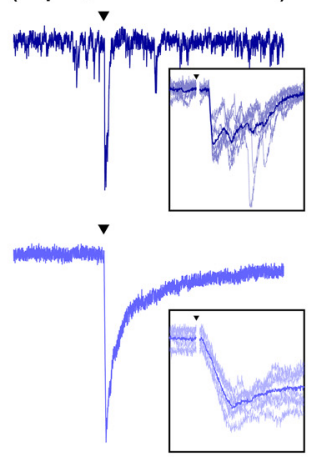

C

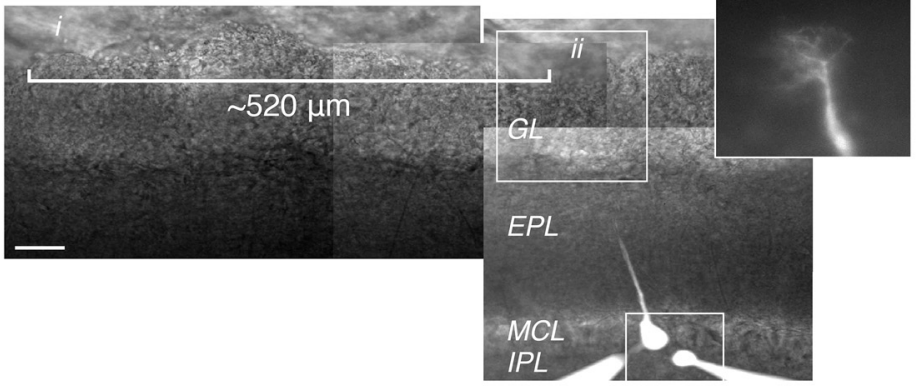

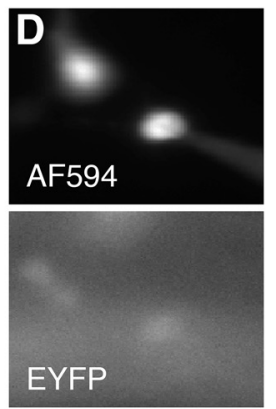
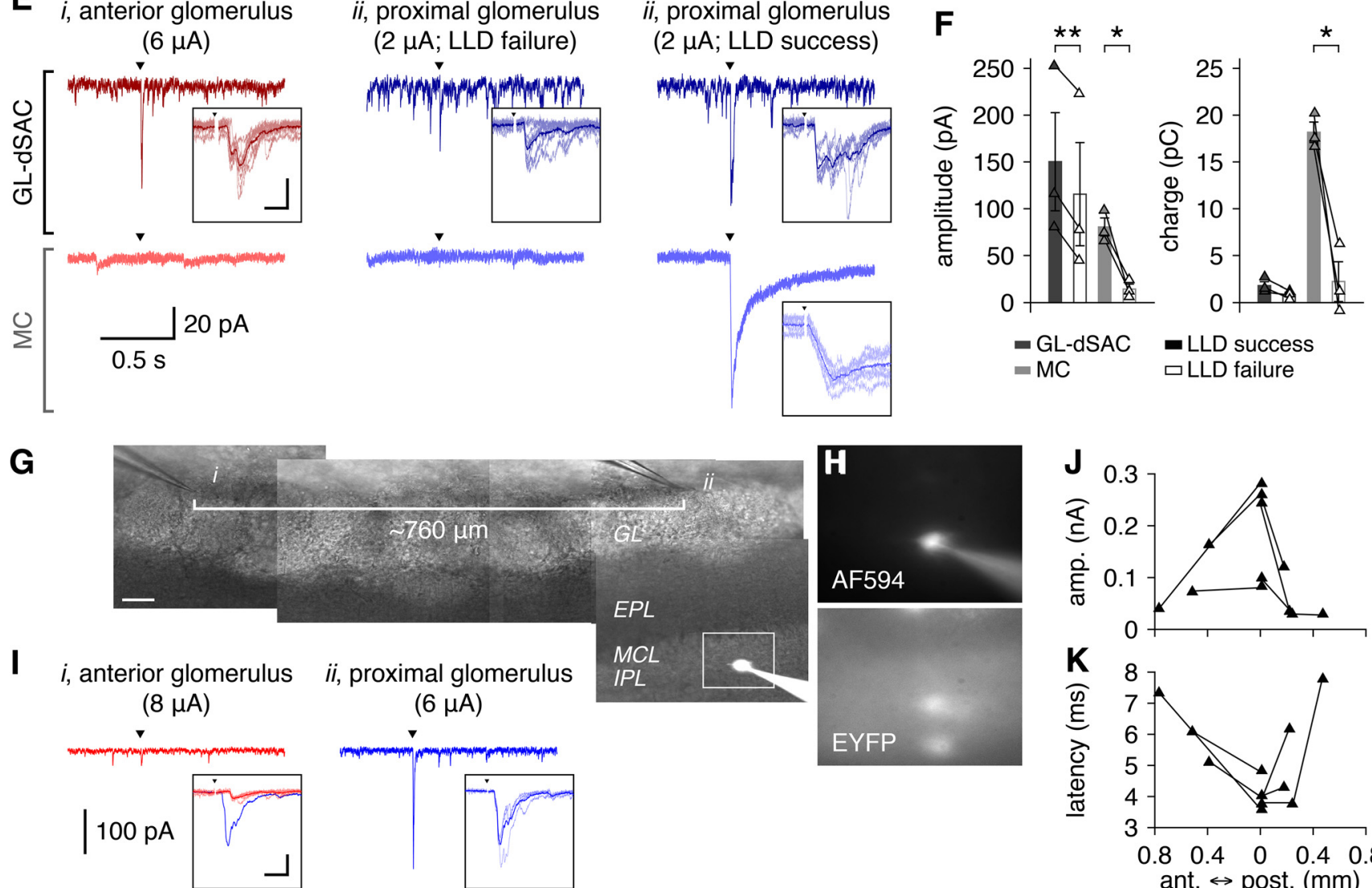

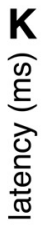

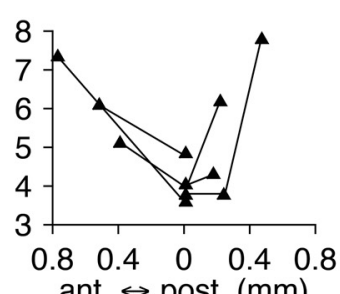

Figure 9. GL-dSACs integrate convergent excitation from several glomeruli. $A$, Excitatory input to pairs of GL-dSACs and nearby MCs was recorded after activation of single nearby or distant glomeruli by OSN stimulation. $\boldsymbol{B}-\boldsymbol{D}$, Representative paired recording of a MC and EYFP-expressing GL-dSAC, with activation of a nearby glomerulus (ii) that contains the MC apical dendritic tuft ( $\boldsymbol{C}$ ) or a glomerulus $\sim 520 \mu \mathrm{m}$ anterior (i). Scale bar, $50 \mu \mathrm{m}$. $\boldsymbol{E}$, Excitatory input to the GL-dSAC/MC pair shown in $\boldsymbol{B}-\boldsymbol{D}$ after OSN stimulation (arrowhead). For nearby glomerulus activation, 0 SNs were stimulated at intensities perithreshold for generating a MCLLD. Results are plotted as in Figure 6 . Inset scale bar, $10 \mathrm{~ms} / 50 \mathrm{pA}$. F, Mean amplitude and charge of excitatory input to $\mathrm{GL}-\mathrm{dSAC} / \mathrm{MC}$ pairs $(n=3)$ on LLD success and LLD failure trials. Excitatory input to GL-dSACs was moderately weaker on LLD failure trials (amplitude: $150.2 \pm 90.8$ vs $115.5 \pm 95.5 \mathrm{pA}$, LLD success vs LLD failure; $p=$ $8.0 \times 10^{-3}$, two-tailed paired $t$ test; charge: $1.8 \pm 0.7$ vs $0.9 \pm 0.4 \mathrm{pC}$, LLD success vs LLD failure; $p=0.09$, two-tailed paired $t$ test), whereas excitatory input to MCs was absent on LLD failure trials (amplitude: $80.5 \pm 16.6$ vs $14.5 \pm 8.7 \mathrm{pA}$, LLD success vs LLD failure; $p=0.035$, two-tailed paired $t$ test; charge: $18.2 \pm 1.9 \mathrm{vs} 2.2 \pm 3.7 \mathrm{pC}$, LLD success vs LLD failure; $p=0.030$, two-tailed paired $t$ test). $\boldsymbol{G}, \boldsymbol{H}$, Representative GL-dSAC recording, with activation of a nearby glomerulus (ii) or a glomerulus $\sim 760 \mu \mathrm{m}$ anterior (i). Scale bar, $50 \mu \mathrm{m}$. $\boldsymbol{I}$, Excitatory input to the cell shown in $\boldsymbol{G}$ and $\boldsymbol{H}$ after $0 S \mathrm{SN}$ stimulation (arrowhead). Inset scale bar, $10 \mathrm{~ms} / 100 \mathrm{pA}$. $\boldsymbol{J}, \boldsymbol{K}$, Summary of the mean amplitude $(\boldsymbol{J})$ and latency $(\boldsymbol{K})$ of excitatory input to $\mathrm{GL}$-dSACs after activation of a single nearby glomerulus and either $1(n=3)$ or 2 distinct $(n=2)$ distant glomeruli.

known to target both glutamatergic and other GABAergic neuron classes. This divergent connectivity profile within the MOB circuit mirrors the divergent connectivity of Martinotti cells (MNCs) and neurogliaform cells (NGFCs) within neocortical circuits (Jiang et al., 2015), suggesting that GL-dSACs, MNCs, and NGFCs may fulfill common functional roles. GLdSACs also share several other features with MNCs and NGFCs. Structurally, both GL-dSACs and MNCs are defined by their superficial axonal projections, and GL-dSACs, MNCs, and superficial NGFCs all arborize their axons across superfi- cial layers (Eyre et al., 2008; Kubota, 2014; Jiang et al., 2015). Functionally, these common axonal structures enable GLdSACs, MNCs, and NGFCs to inhibit the distal apical dendrites of principal TCs and pyramidal cells (Kubota, 2014; Jiang et al., 2015) and thereby regulate the integration of sensory input in vivo (Murayama et al., 2009). In addition, NGFCs also express nAChRs and respond to endogenous ACh release, suggesting that both NGFCs and GL-dSACs rapidly modulate circuit activity across distinct brain states (Wester and McBain, 2014; Letzkus et al., 2015). 
A
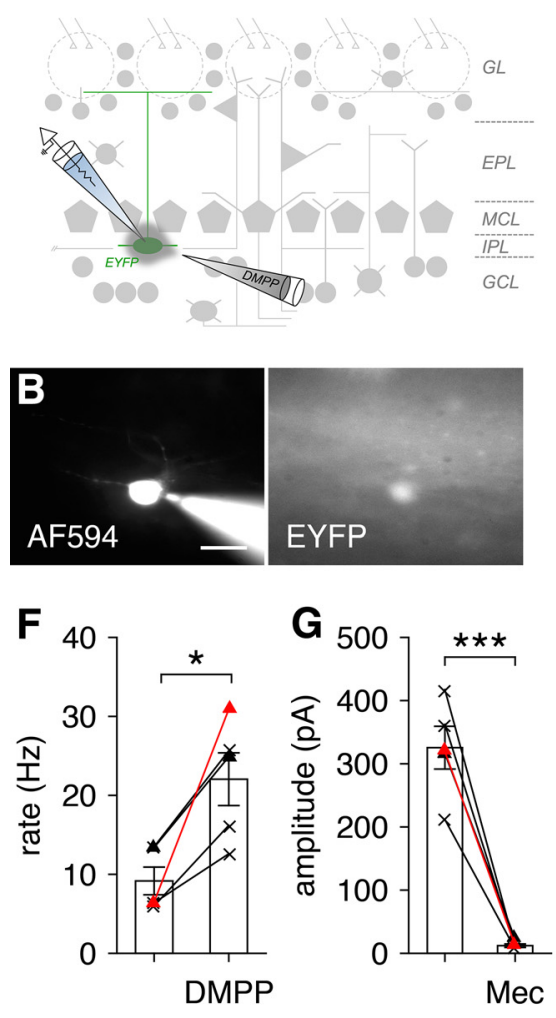

$\Delta$ control $\times$ NBQX/AP5/GBZ (N/A/G)
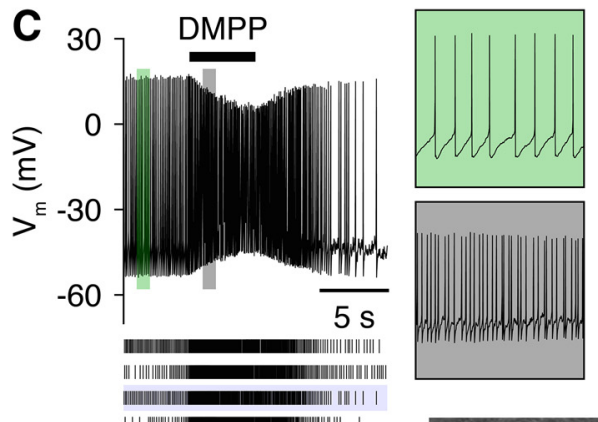

H

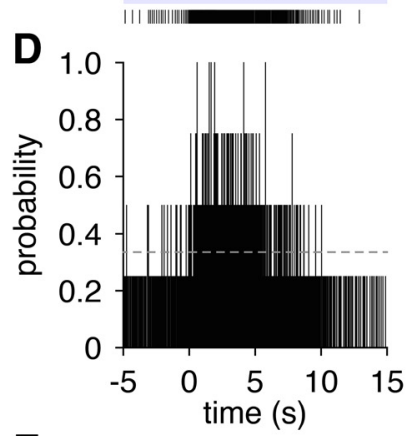

E

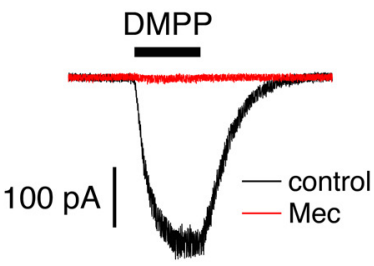

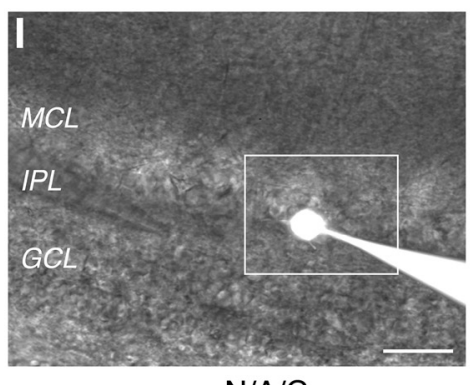
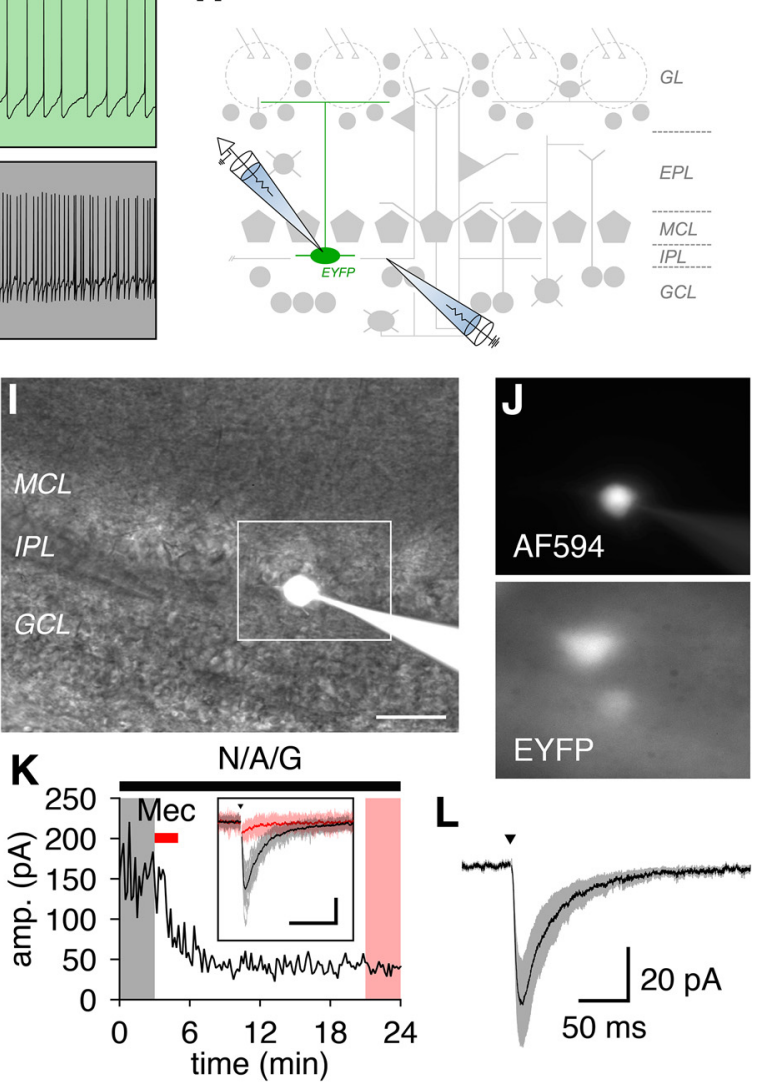

EYFP

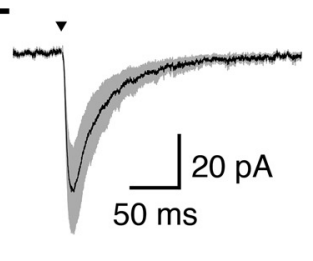

Figure 10. GL-dSACs exhibit nicotinic responses to endogenous ACh release. $\boldsymbol{A}, \mathrm{GL}-\mathrm{dSAC}$ activity was recorded during focal application of broad-spectrum nicotinic agonist DMPP. $\boldsymbol{B}$, Whole-cell recording of a representative EYFP-expressing GL-dSAC. Scale bar, $20 \mu \mathrm{m}$. $\boldsymbol{C}, \boldsymbol{D}$, Focal application of DMPP consistently increased the spontaneous firing rate of the cell shown in $\boldsymbol{B}$. Results are plotted as in Figure 6. Insets, Magnification of 1-s-long periods of firing before and during DMPP appplication. $\boldsymbol{E}$, Voltage-clamp recording of the mean inward current evoked by DMPP in the cell shown in B. Bath application of Mec abolished the inward current. $\boldsymbol{F}$, DMPP application significantly increased GL-dSAC spontaneous firing rates $(9.2 \pm 3.9$ vs $22.0 \pm 7.5 \mathrm{~Hz}$, before vs during DMPP application; $n=5 ; p=0.013$, two-tailed paired $t$ test). Red points denote cell shown in $\boldsymbol{B}-\boldsymbol{E}$. $\boldsymbol{G}$, Mec blocked the DMPP-evoked inward current in GL-dSACs, leading to a significant reduction in current amplitude ( $325.5 \pm 75.6$ vs $12.0 \pm 6.1 \mathrm{pA}$, before vs after Mec application; $n=5 ; p=7.3 \times 10^{-4}$, two-tailed paired $t$ test). Red points denote cell shown in $\boldsymbol{B}-\boldsymbol{E}$. $\boldsymbol{H}$, Synaptic input to GL-dSACs was recorded while stimulating the IPL and blocking glutamatergic and GABAergic receptors. I, J, Whole-cell recording of a representative EYFP-expressing GL-dSAC. Scale bar, $20 \mu \mathrm{m}$. $\boldsymbol{K}$, IPL stimulation (10 $\mu \mathrm{A})$ in the presence of NBQX/AP5/GBZ evoked a robust EPSC in the cell shown in $\boldsymbol{I}$ and $\boldsymbol{J}$ that was abolished rapidly by Mec application. Inset, Mean synaptic response evoked by IPL stimulation (arrowhead) before and after Mec application (scale bar, $100 \mathrm{~ms} / 50 \mathrm{pA}$ ). Individual trials are shown in lighter colors. L, Mean postsynaptic nicotinic current recorded in GL-dSACs (peak: $57.7 \pm 38.8 \mathrm{pA}$; decay constant: $29.2 \pm 7.2 \mathrm{~ms} ; n=6$ ) after IPL stimulation (arrowhead; $10-15 \mu \mathrm{A}$ ) calculated by subtracting the mean current after Mec application from the mean current before Mec application (in the presence of NBQX/AP5/GBZ).

Interestingly, both GL-dSACs and NGFCs are also capable of mediating long-lasting inhibition of principal neurons, though likely via distinct mechanisms. Specifically, NGFCs mediate graded long-lasting inhibition of both principal neurons and interneurons by unitary volume transmission of GABA (Tamás et al., 2003). In contrast, synchronous GL-dSAC activation evokes all-or-none long-lasting inhibition selectively in TCs. We hypothesize that synchronous release of GABA from GL-dSACs excites at least a subset of PGCs with depolarized $\mathrm{Cl}^{-}$reversal potentials (Smith and Jahr, 2002; Parsa et al., 2015) triggering regenerative GABA-induced GABA release (Parsa et al., 2015) that targets the TC apical dendritic tuft. Future work investigating functional PGC subclasses and M/TC-PGC connectivity (Najac et al., 2015) will be critical in evaluating this proposed mechanism.

GL-dSACs also share several features with somatostatinexpressing hippocampal lacunosum-moleculare-projecting oriens (O-LM) cells, which also express chrna2 (Ishii et al., 2005; Leão et al., 2012). In particular, O-LM cells exhibit horizontal cell morphologies and arborize their axons across the most distal input layer of the CA1 circuit. Within this distal layer, O-LM cell axons inhibit the apical dendritic tufts of pyramidal cells to regulate the integration of sensory input in vivo (Lovett-Barron et al., 2014). Moreover, O-LM cells express non-desensitizing nAChRs (Jia et al., 2009) and respond to endogenous ACh release (Leão et al., 2012).

In total, this conservation of structure and function across GL-dSACs, MNCs, NGFCs, and O-LM cells suggests a core set of emergent interneuron features critical for regulating activity and sensory processing across distinct neocortical, hippocampal, and MOB circuits.

\section{Temporal patterning of MOB activity}

Theta-frequency patterning of MOB activity emerges from both odor sampling via sniffing and the spontaneous bursting of ETCs (Wachowiak, 2011) and critically regulates olfactory processing. Changes in theta-frequency sniffing rates can modulate the activation of both OSNs and M/TCs profoundly (Wachowiak, 2011). Moreover, theta-frequency patterning provides a temporal reference for sensory encoding by M/TC activity (Fukunaga et al., 2012, 2014). Here, we now demonstrate that GL-dSACs fire spontaneously at theta frequencies and respond to sensory acti- 


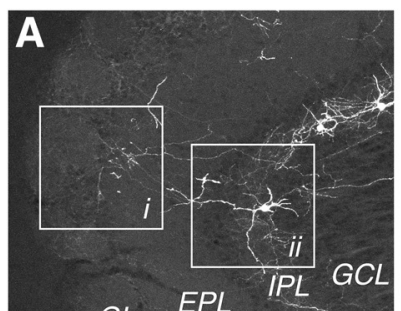

GL

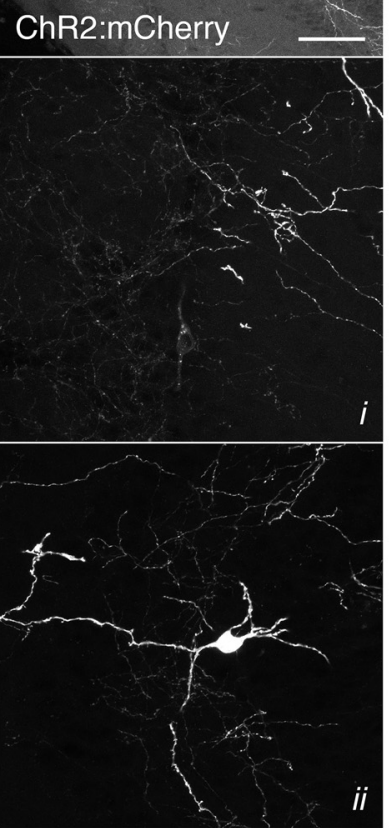

I

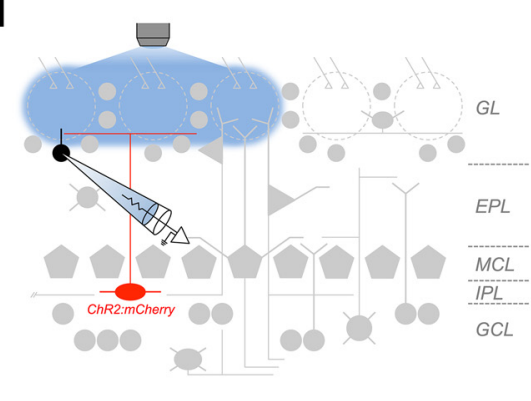

J

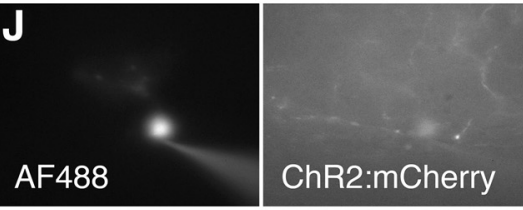

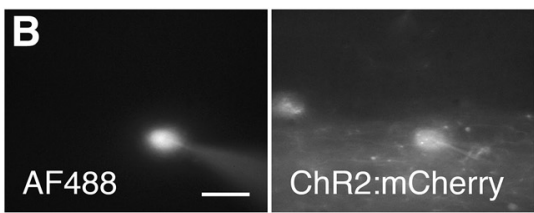

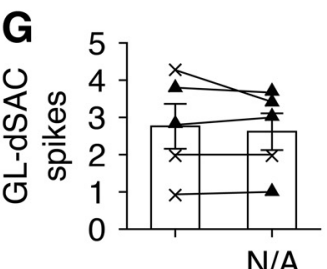

E
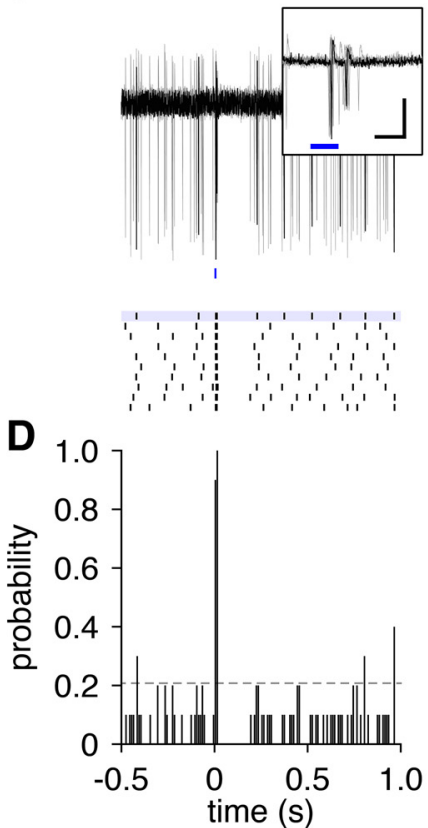

$-60$
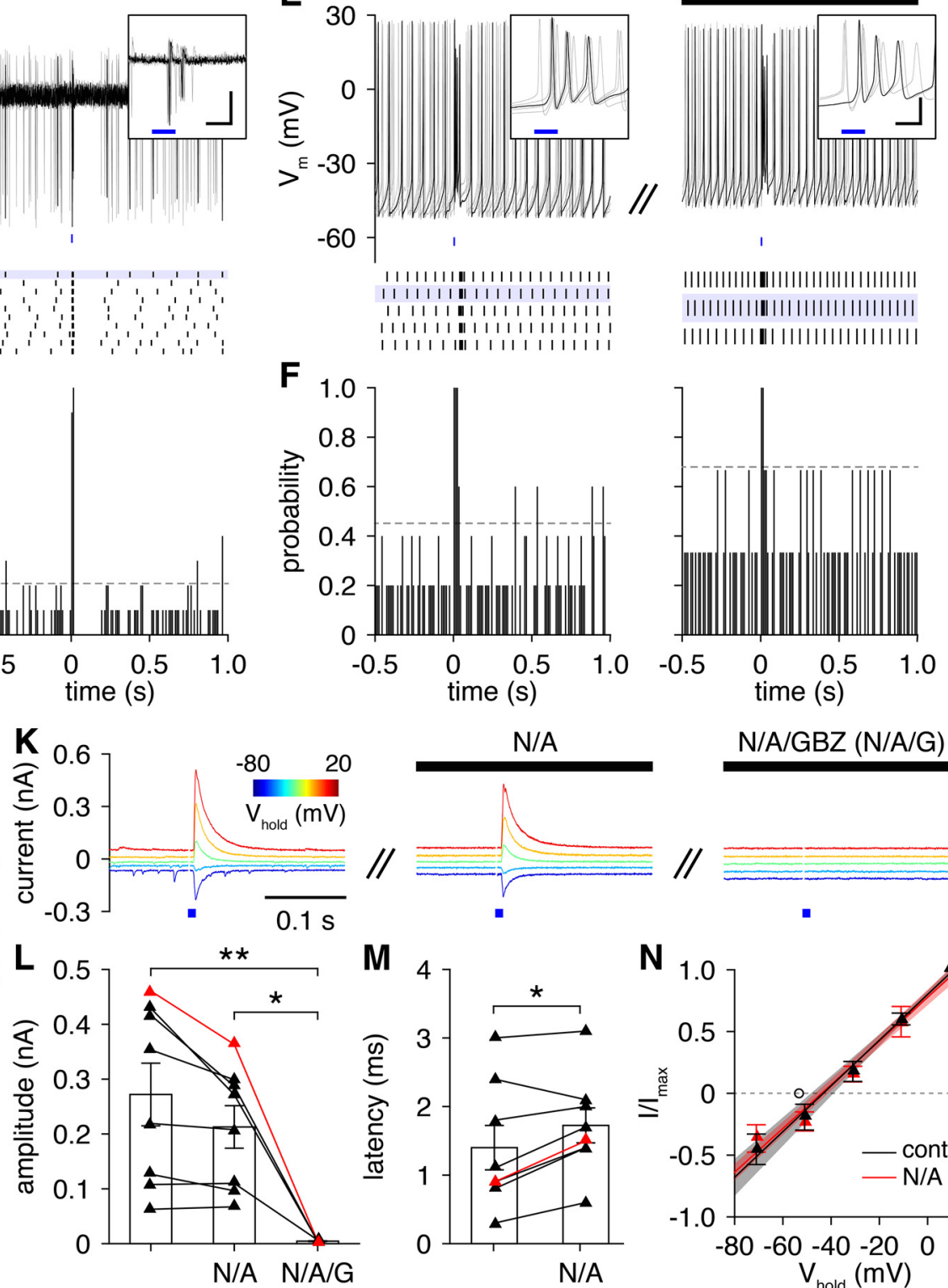

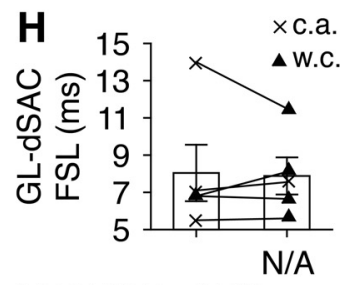

NBQX/AP5 (N/A)

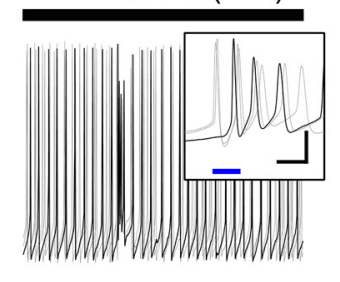

|||||||||||||||||||||||||||||||||||| IIIIIIIIIIIIIIIIIIIII|IIIIIII |l||||||||||||||||||||||||||||

$\mathrm{N} / \mathrm{A} / \mathrm{GBZ}(\mathrm{N} / \mathrm{A} / \mathrm{G})$
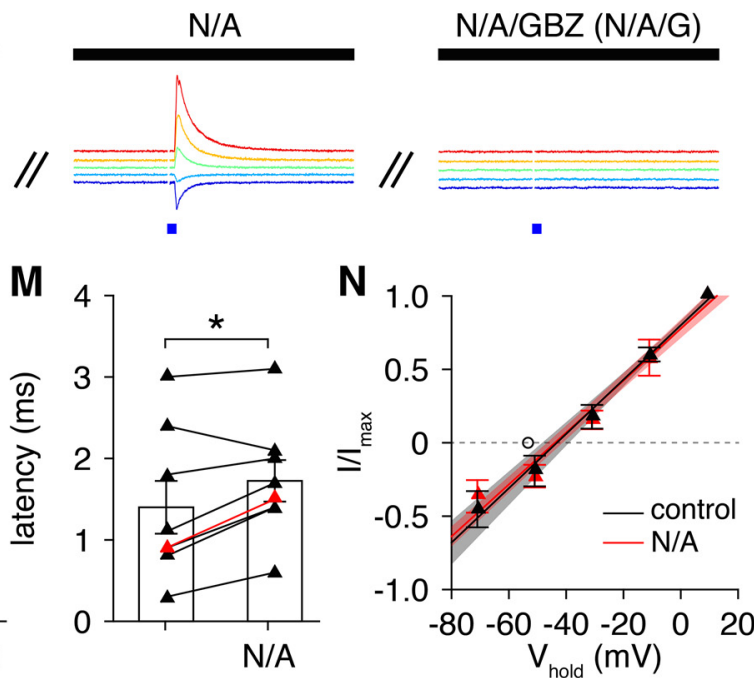

Figure 11. GL-dSACs innervate PGCS. A, Cre-dependent ChR2:mCherry expression. Scale bar, $100 \mu \mathrm{m} . \boldsymbol{B}-\boldsymbol{D}$, Representative cell-attached recording (and post hoc cell fill; scale bar, $20 \mu \mathrm{m}$ ) of a GL-dSAC after brief photostimulation ( $10 \mathrm{~ms}$; blue line). Results plotted as in Figure 6. Inset scale bar, $10 \mathrm{~ms} / 50 \mathrm{pA}$. E, $\boldsymbol{F}$, Same as $C$ and $\boldsymbol{D}$ for whole-cell recording from a different GL-dSAC before and after NBQX/AP5 application. Inset scale bar, $10 \mathrm{~ms} / 20 \mathrm{mV} . \mathbf{G}, \boldsymbol{H}$, Photostimulation evoked $1-4$ action potentials in GL-dSACs independent of glutamatergic transmission (spikes: $2.8 \pm 1.3 \mathrm{vs} 2.6 \pm$ 1.1, before vs after NBQX/AP5 application; $n=5 ; p=0.48$, two-tailed paired $t$ test; first-spike latency, FSL; $8.0 \pm 3.4$ vs $7.9 \pm 2.2 \mathrm{~ms}$, before vs after NBQX/AP5 application; $n=5 ; p=1.0$, two-sided Wilcoxon signed-rank test). w.c., Whole-cell recordings; c.a., cell-attached recordings. $\boldsymbol{I}$, Synaptic input to PGCs was recorded after GL-dSAC photostimulation. $\boldsymbol{J}, \boldsymbol{K}$, Representative whole-cell recording of photostimulation-evoked input to a PGC across various holding potentials (corrected for liquid junction potential). NBQX/AP5 application blocked sEPSCs but had no effect on evoked input, whereas GBZ application abolished the evoked input. $\boldsymbol{L}, \boldsymbol{M}, \mathrm{Amplitude}(\boldsymbol{L})$ and latency $(\boldsymbol{M})$ of GL-dSAC-mediated input to PGCs. Application of GBZ but not NBQX/AP5 blocked the evoked input to PGCs $\left(L ; 0.27 \pm 0.16, n=8\right.$, vs $0.21 \pm 0.11, n=8$, vs $0.00 \pm 0.00 \mathrm{nA}, n=4$, control vs NBQX/AP5 application vs NBQX/AP5/GBZ application; $p=9.4 \times 10^{-3}$, one-way ANOVA; control vs NBQX/AP5/GBZ application, $p=7.5 \times 10^{-3}, \mathrm{NBQX} / \mathrm{AP5}$ application vs NBQX/AP5/GBZ application, $p=0.037$, post hoc Tukey-Kramer). NBQX/AP5 application minimally increased the latency of evoked input $(\boldsymbol{M} ; 1.4 \pm 0.9, n=8$, vs $1.7 \pm 0.7 \mathrm{~ms}, n=8$, before vs after NBQX/AP5 application; $p=0.024$, two-tailed paired $t$ test). Red points denote cell shown in $\boldsymbol{J}$ and $\boldsymbol{K}$. $\boldsymbol{N}$, Current-voltage relationship (corrected for liquid junction potential) of the evoked input to $\mathrm{PGCS}(n=5)$ was independent of glutamatergic transmission $(-44.6 \pm 7.8 \mathrm{vs}-44.0 \pm 5.0 \mathrm{mV}$ without or with NBQX/AP5 application; $p=0.69$, two-sided Wilcoxon rank-sum test). The reversal potential of evoked input to PGCs $(n=5)$ was significantly more depolarized than the reversal potential of evoked input to $\operatorname{ETCs}(n=4)$ and ETC-like sTCs $(n=4)$ (open circle) $(-44.6 \pm 7.8 \mathrm{vs}-53.3 \pm 6.9 \mathrm{mV} ; p=0.029$, one-tailed unpaired $t$ test). 

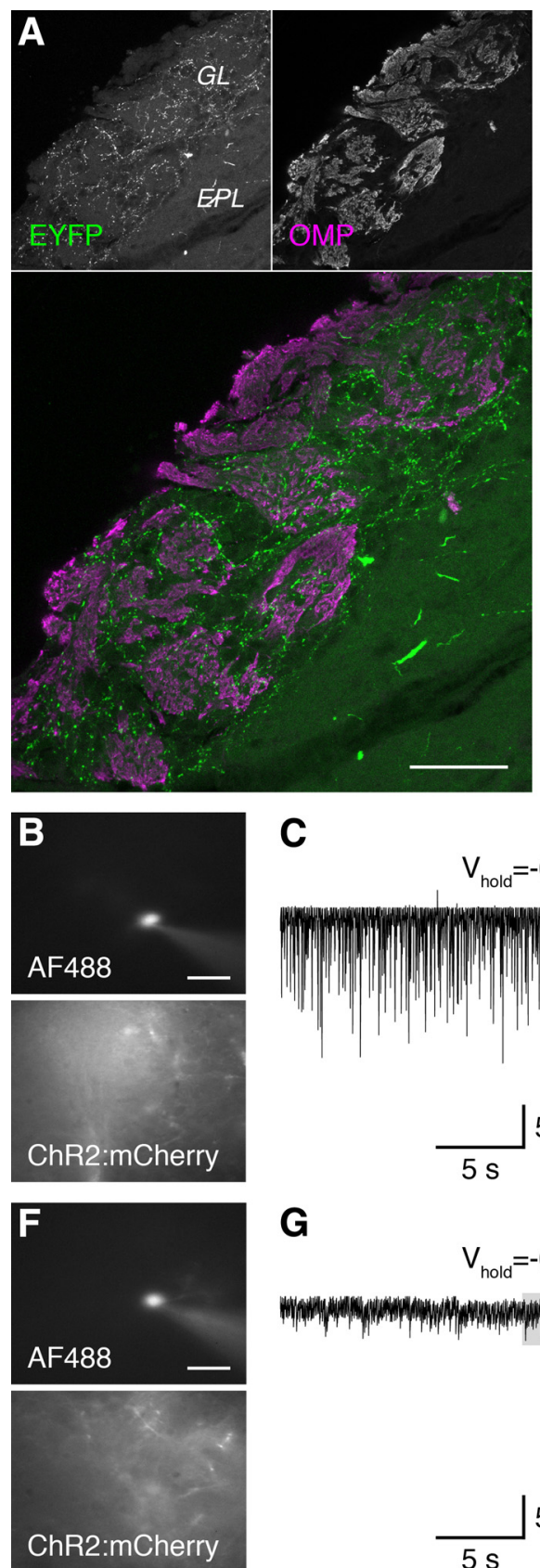

C

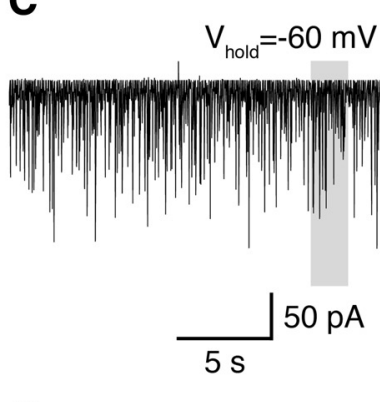

G
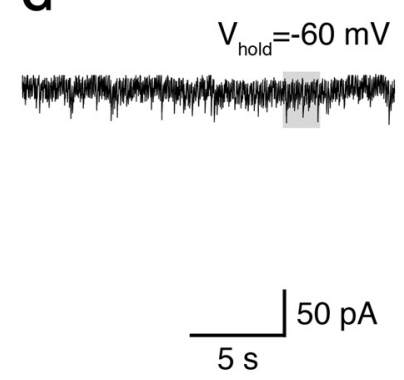
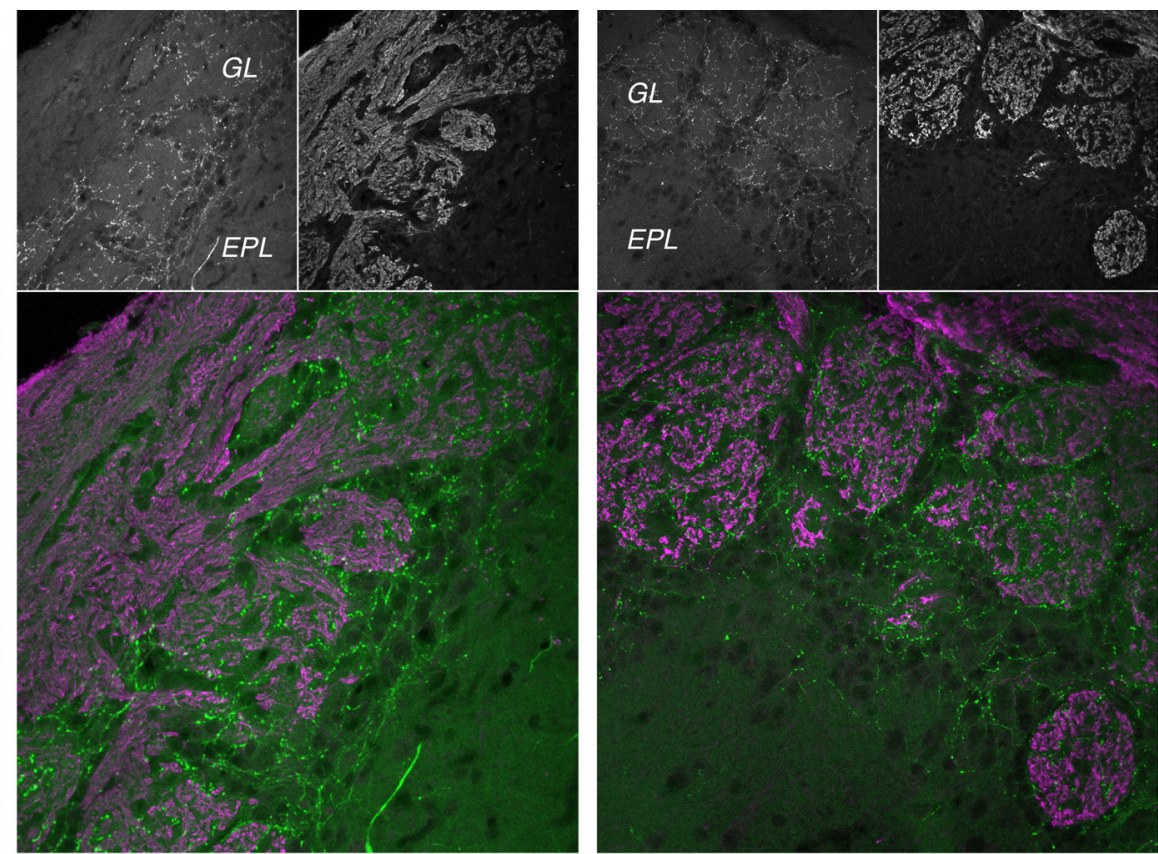

D

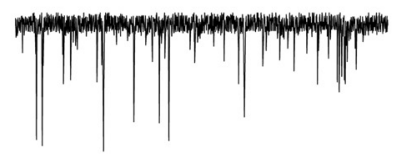

E

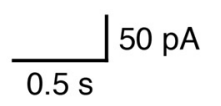

H
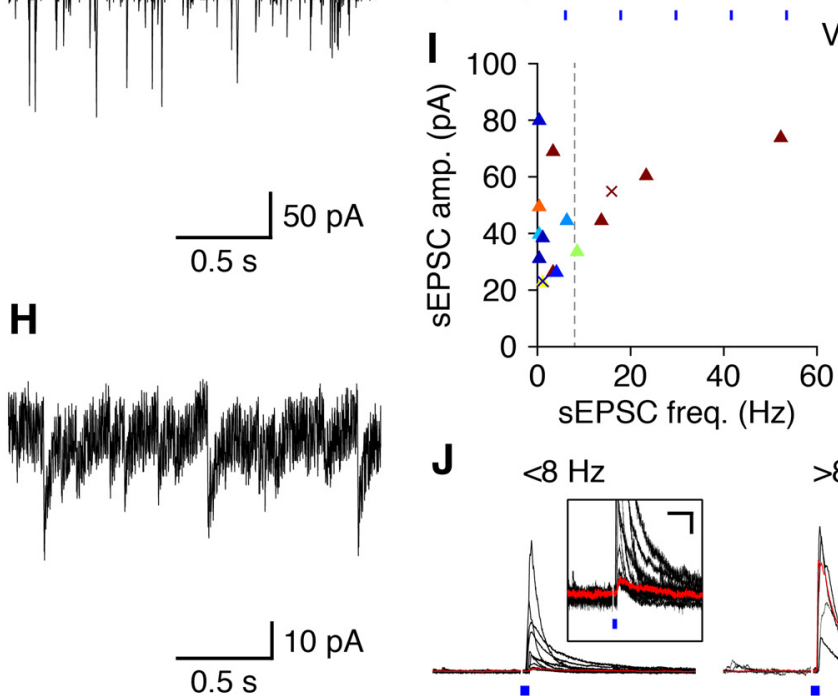

$>8 \mathrm{~Hz}$

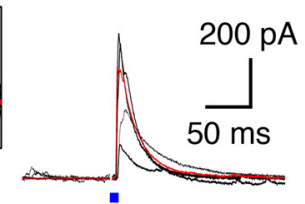

Figure 12. GL-dSACs innervate multiple functional PGC subclasses. A, EYFP-expressing GL-dSAC axonal boutons in Chrna2-Cre/Ai3 mice predominantly innervate OMP-negative glomerular compartments. Examples from three mice are shown. Scale bar, $50 \mu \mathrm{m}$. B, Whole-cell recording of a representative putative CB-PGC. Scale bar, $20 \mu \mathrm{m}$. $\boldsymbol{C}$, Spontaneous excitatory synaptic input to the cell shown in $\boldsymbol{B}$ exhibiting high sEPSC frequencies and amplitudes. D, Magnification of highlighted region in $\boldsymbol{C}$. $\boldsymbol{E}$, Repetitive $5 \mathrm{~Hz} \mathrm{GL}$-dSAC photostimulation evoked highly reliable and minimally depressing postsynaptic responses in the putative CB-PGC shown in $\boldsymbol{B}$. Results are plotted as in Figure 6 . $\boldsymbol{F}-\boldsymbol{H}$, Same as $\boldsymbol{B}$ - $\boldsymbol{D}$ for a representative putative CR-PGC exhibiting low sEPSC frequencies and amplitudes. $I$, Distribution of PGC sEPSC amplitudes and frequencies color coded by the amplitude of postsynaptic input evoked by GL-dSAC photostimulation. Dashed line at 8 Hz separates putative CR-PGCS ( $<8 \mathrm{~Hz}$ ) from putative CB-PGCs ( $>8 \mathrm{~Hz}$ ). Crosses correspond to the putative CB-PGC and CR-PGC shown in $\boldsymbol{B}-\boldsymbol{D}$ and $\boldsymbol{F}-\boldsymbol{H}$, respectively. $\boldsymbol{J}$, Mean postsynaptic input evoked by GL-dSAC photostimulation (blue square) in putative CR-PGCs and CB-PGCs. Inset: magnification of synaptic response in putative CR-PGCs (scale bar, 50 ms/10 pA). Red traces correspond to cells shown in $\boldsymbol{B}-\boldsymbol{H}$.

vation of the MOB circuit with a change in spike timing. These results suggest that GL-dSACs provide an additional source of theta-frequency patterning intrinsic to the MOB. Moreover, the convergent ETC input to GL-dSACs and divergent GL-dSAC output to ETCs suggests that coherent ETC and GL-dSAC activity may be critical to long-range coordination of theta-frequency activity (Kay and Lazzara, 2010).

Mechanistically, the highly precise spike timing response of GL-dSACs to sensory activation of the MOB circuit likely emerges from three functional features. First, excitatory input to GL-dSACs following glomerular activation is short in duration, in strong contrast to the LLDs observed in M/TCs (Carlson et al., 2000; De Saint Jan et al., 2009; Gire and Schoppa, 2009; Burton and Urban, 2014) and the asynchronous excitation observed in GCs (Burton and Urban, 2015; Geramita et al., 2016). Second, GL-dSAC firing after glomerular activation is constrained to a window of opportunity by reliable feedforward inhibition (Pinto et al., 1996; Pouille and 

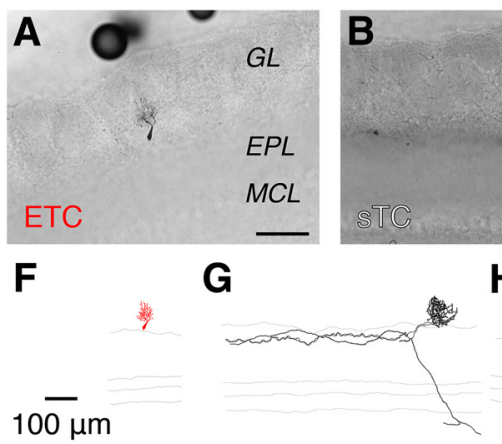

$\mathrm{K}$
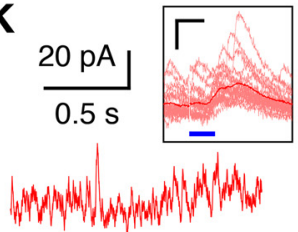

, $\mathrm{V}_{\text {hold }}=+10 \mathrm{mV}$
L

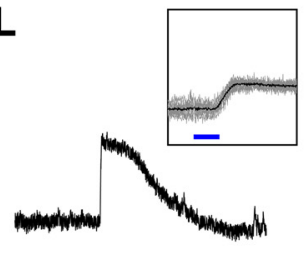

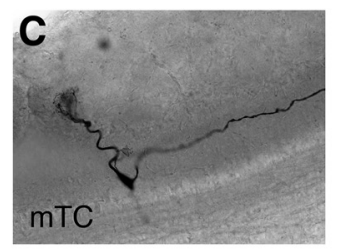

I
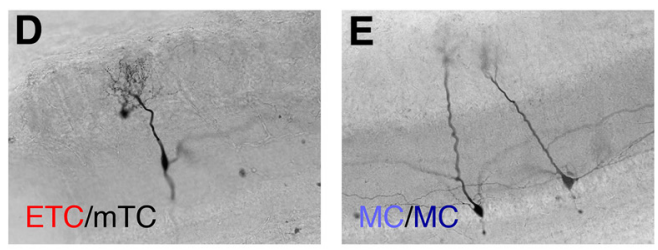

J

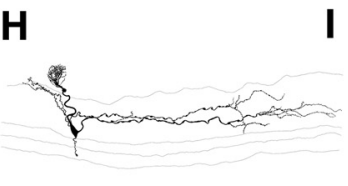

M

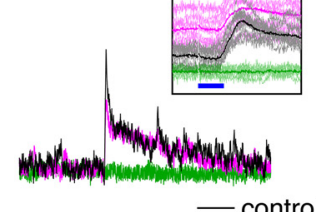

N

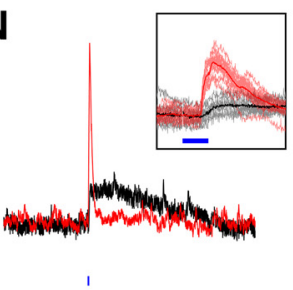

0
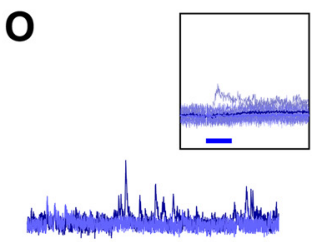

$$
V_{\text {hold }}=-60 \mathrm{mV}
$$

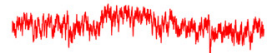
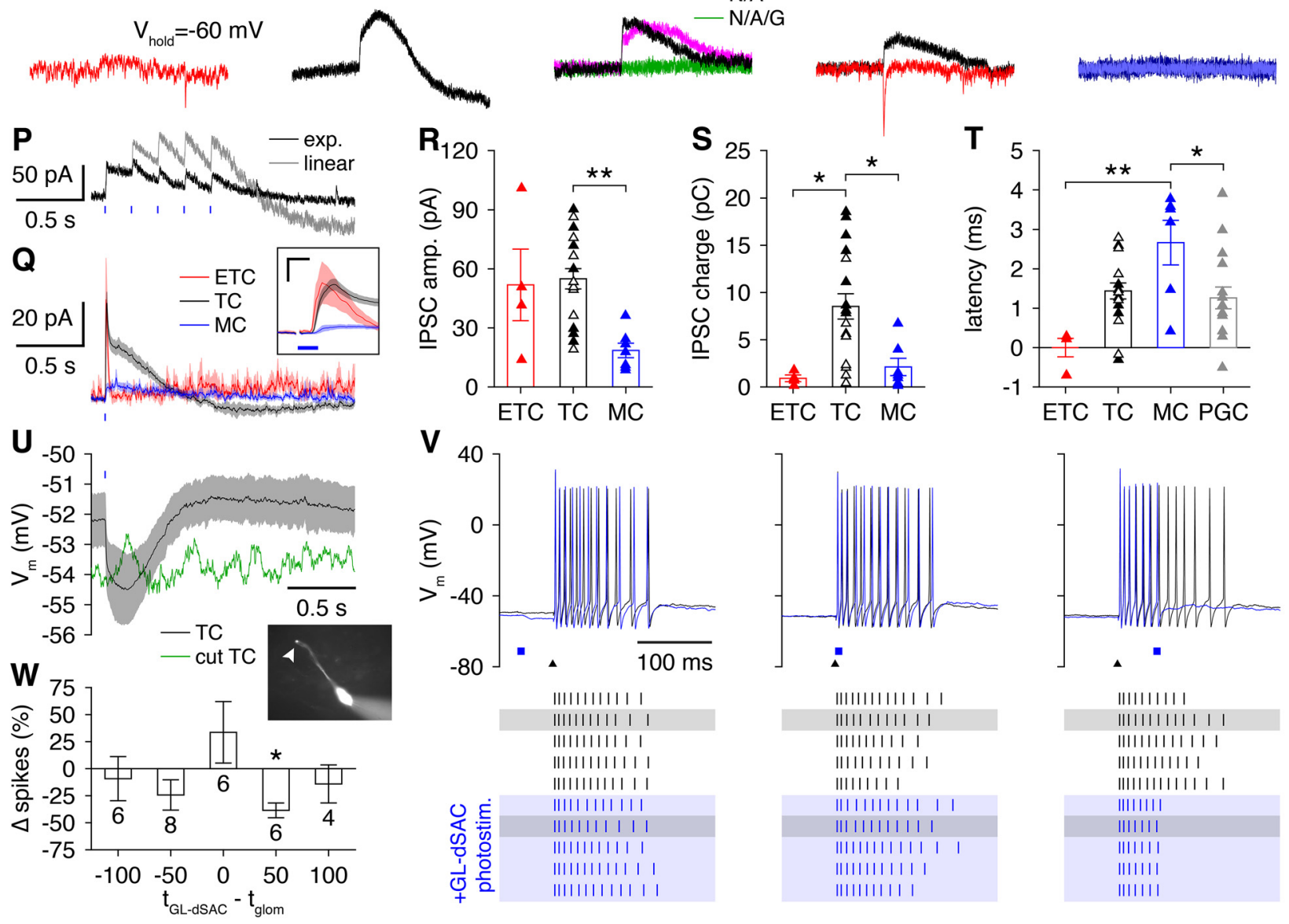

IIIIIIIIIIIII | |

IIIIIIIIII I I

IIIIIIIII I

IIIIIIII। |

IIIIIIII I

IIIIIIIIIII | |

IIIIIIIIIIII

|1111111111। |

|IIIIIIIII।

||IIIIII।| |
|IIIIIIIII

IIIIIIIIIII I |

IIIIIIIIIIII I

|IIIIIIIIII

IIIIIIIIIIII

|IIIIIII

IIIIIII

IIIIIII

IIIIIII

IIIIIII

Figure 13. GL-dSACs inhibit ETCs and TCs and can drive long-lasting TC inhibition. $\boldsymbol{A}-\boldsymbol{0}$, Morphology $(\boldsymbol{A}-\boldsymbol{J}$; scale bar, $100 \boldsymbol{\mu m})$ and voltage-clamp recordings of postsynaptic currents $(\boldsymbol{K}-\boldsymbol{0})$ from a representative ETC $(\boldsymbol{A}, \boldsymbol{F}, \boldsymbol{K}), \operatorname{sTC}(\boldsymbol{B}, \boldsymbol{G}, \boldsymbol{L}), \mathrm{mTC}(\boldsymbol{C}, \boldsymbol{H}, \boldsymbol{M}), \mathrm{ETC} / \mathrm{mTC}$ pair $(\boldsymbol{D}, \boldsymbol{I}, \boldsymbol{N})$, and MC/MC pair $(\boldsymbol{E}, \boldsymbol{J}, \boldsymbol{0})$ after brief GL-dSAC photostimulation (10 ms; blue line). Results are plotted as in Figure 6. Inset scale bar, $10 \mathrm{~ms} / 50 \mathrm{pA}$. $\boldsymbol{P}$, Repetitive $5 \mathrm{~Hz} \mathrm{GL}$-dSAC photostimulation evoked strongly depressing postsynaptic responses (exp.) $\left(V_{\text {hold }}=+10 \mathrm{mV}\right.$ ) in the sTC shown in $\boldsymbol{B}$ and $\boldsymbol{G}$. Linear summation of the responses to isolated GL-dSAC photostimulation $(\boldsymbol{L})$ is shown for comparison. $\boldsymbol{Q}$, Mean postsynaptic currents $\left(V_{\text {hold }}=+10 \mathrm{mV}\right)$ evoked by $G \mathrm{~L}-\mathrm{dSAC}$ photostimulation in ETCs $(n=$ 4), TCs $(n=18)$, and MCs $(n=7)$. Inset scale bar, $10 \mathrm{~ms} / 20 \mathrm{pA}$. $\boldsymbol{R}-\boldsymbol{T}$, Amplitude $(\boldsymbol{R})$, charge (S), and latency $(\boldsymbol{T})$ of inhibitory synaptic input to ETCs, TCs (including sTCs, white triangles, and mTCs, black triangles,), and MCs evoked by GL-dSAC photostimulation. TCs exhibited significantly larger amplitudes than $\mathrm{MCs}(\boldsymbol{R} ; 51.9 \pm 36.3, n=4, \mathrm{vs} 55.0 \pm 21.9, n=18, \mathrm{vs} 18.6 \pm 9.7 \mathrm{pA}, n=7$, ETC vs TC vs MC; $p=3.6 \times 10^{-3}$, one-way ANOVA; TC vs MC, $p=2.8 \times 10^{-3}$, post hoc Tukey-Kramer) and greater charge transferred than ETCs and MCs ( $; ; 0.9 \pm 0.7, n=4$, vs $8.5 \pm 5.7, n=$ 18 , vs $2.1 \pm 2.4 \mathrm{pC}, n=7$, ETC vs TC vs MC; $p=1.6 \times 10^{-3}$, Kruskal - Wallis test; TC vs ETC, $p=0.015$, TC vs MC, $p=0.014$, post hoc Tukey-Kramer). MC latencies were significantly longer than ETC and PGC latencies $\left(T ; 0.0 \pm 0.5, n=4\right.$, vs $1.4 \pm 0.9, n=18$, vs $2.7 \pm 1.4, n=6$, vs $1.3 \pm 1.1 \mathrm{~ms}, n=16$, ETC vs TC vs MC vs PGC; $p=2.3 \times 10^{-3}$, one-way ANOVA; MC vsETC, $p=1.2 \times$ $10^{-3}, \mathrm{MC}$ vs PGC, $p=0.031$, post hoc Tukey-Kramer). $\boldsymbol{U}$, Mean GL-dSAC-mediated TC hyperpolarization ( $n=26$ ) compared with a representative TC with truncated apical dendrite (inset). $\boldsymbol{V}$, Whole-cell recording of a representative TC after GL-dSAC photostimulation (blue square) before (left), during (center), or after (right) 0SN stimulation (black triangle). W, GL-dSAC photostimulation $50 \mathrm{~ms}$ after $\mathrm{OSN}$ stimulation significantly reduced sensory-evoked TC firing ( $p=0.012$, two-tailed $t$ test with Bonferroni correction for multiple comparisons). Column numbers denote TCs recorded. 
A
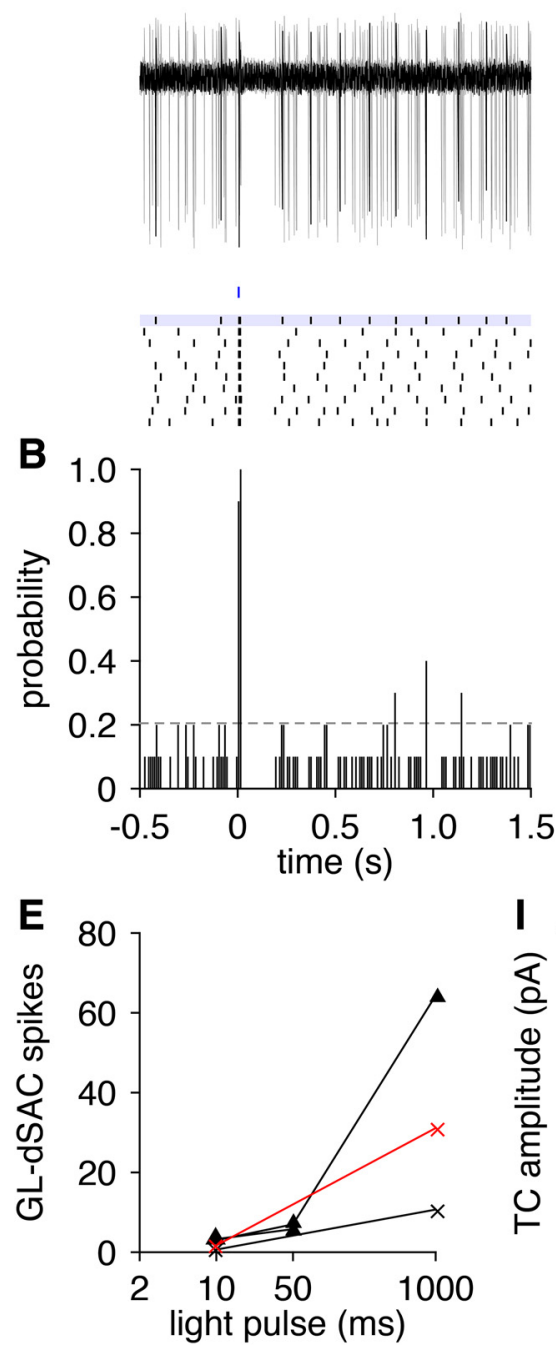

C

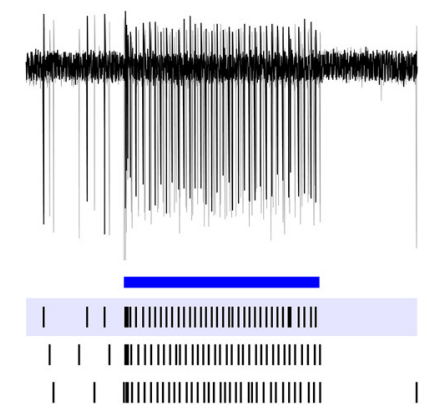

D

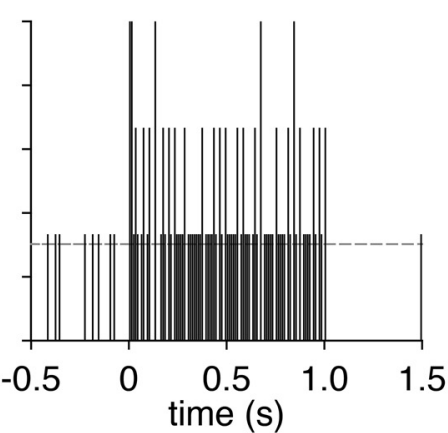

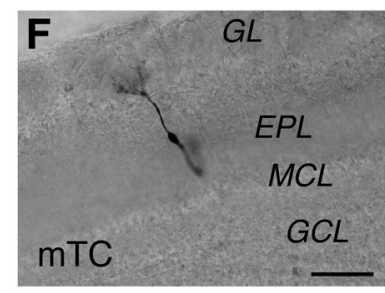

G
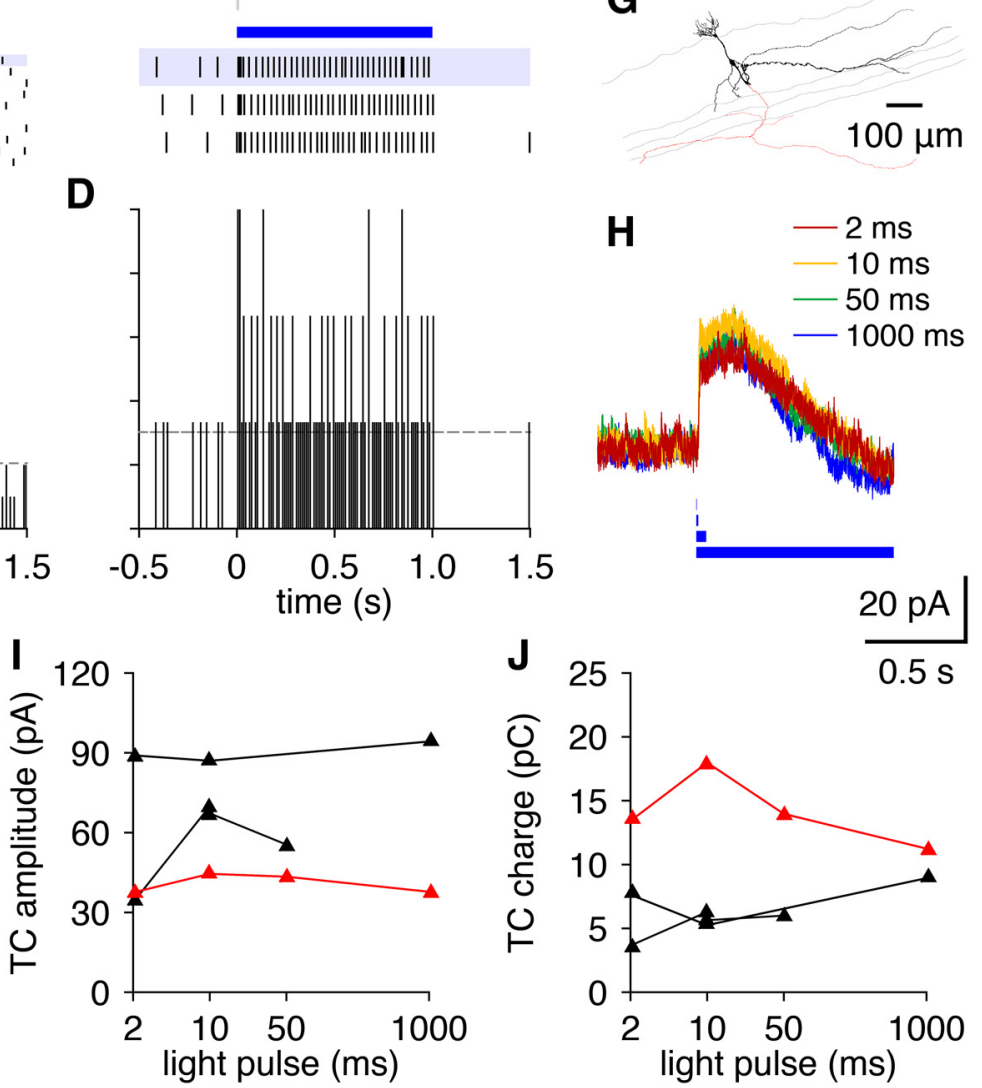

Figure 14. GL-dSAC-mediated long-lasting TC inhibition is all-or-none. $\boldsymbol{A}, \boldsymbol{B}$, Brief photostimulation (10 ms; blue line) evoked one or two action potentials in cell-attached recordings from GL-dSACs expressing ChR2:mCherry. Same cell as in Figure 11B. Results are plotted as in Figure 6. C, D, Same as $\boldsymbol{A}$ and $\boldsymbol{B}$ for prolonged photostimulation (1 s). $\boldsymbol{E}$, Increasing photostimulation duration increased the number of evoked spikes in GL-dSACs expressing ChR2:mCherry. Red points denote cell shown in $\boldsymbol{A}-\boldsymbol{D}$. $\boldsymbol{F}, \boldsymbol{G}$, Neurobiotin fill $(\boldsymbol{F})$ and reconstructed morphology ( $\boldsymbol{G}$ ) of a representative TC. Scale bar, $100 \mu \mathrm{m}$. $\boldsymbol{H}$, Whole-cell voltage-clamp recording of the mean postsynaptic currents $\left(V_{\text {hold }}=+10 \mathrm{mV}\right)$ in the cell shown in $\boldsymbol{F}$ and $\boldsymbol{G}$ evoked by varying-duration photostimulation of GL-dSACs expressing ChR2:mCherry. $\boldsymbol{I}, \boldsymbol{J}$, Amplitude $(\boldsymbol{I})$ and charge $(\boldsymbol{J})$ of inhibitory synaptic input to TCs evoked by varying duration photostimulation of GL-dSACs. In contrast to the increasing number of spikes evoked in GL-dSACs with increasing photostimulation duration (E), inhibitory synaptic input to TCs occurred in an all-or-none manner. Red points denote cell shown in $\boldsymbol{F}$ - $\boldsymbol{H}$.

Scanziani, 2001) mediated by unknown interneurons. Third, dSACs express $\mathrm{K}_{\mathrm{V}} 3.1 \mathrm{~b}$ (Eyre et al., 2009), a high-voltageactivated potassium channel known to accelerate EPSP decay in other interneurons critically involved in precise temporal patterning of network activity (Hu et al., 2014).

\section{Long-range coordination of glomerular activity}

The axons of ETCs connected to mirror symmetric glomeruli in each MOB project reciprocally and selectively to the IPL/sGCL directly beneath the isofunctional glomerulus (Belluscio et al., 2002; Lodovichi et al., 2003), where they synapse exclusively onto GABAergic dendrites (Liu and Shipley, 1994). Despite this anatomical and synaptic selectivity, however, the cellular targets and function of ETC intrabulbar projections remain unknown.

Here, we now hypothesize that ETC intrabulbar projections target GL-dSACs specifically. Although speculative, this hypothesized circuitry provides a parsimonious account of the anatomic and synaptic selectivity of ETC intrabulbar projec- tions and is further consistent with the widespread ETC-toGL-dSAC synaptic connectivity that our results identify. Moreover, this hypothesized circuitry provides a plausible mechanism for the odor-concentration-dependent temporal coordination of mirror-symmetric glomerular microcircuit activity (Zhou and Belluscio, 2012). Interestingly, ETC intrabulbar projections also exhibit pronounced experiencedependent plasticity throughout life (Marks et al., 2006; Cummings and Belluscio, 2010), strongly motivating future research into GL-dSAC plasticity.

\section{References}

Belluscio L, Lodovichi C, Feinstein P, Mombaerts P, Katz LC (2002) Odorant receptors instruct functional circuitry in the mouse olfactory bulb. Nature 419:296-300. CrossRef Medline

Boyd AM, Sturgill JF, Poo C, Isaacson JS (2012) Cortical feedback control of olfactory bulb circuits. Neuron 76:1161-1174. CrossRef Medline

Burton SD, Urban NN (2014) Greater excitability and firing irregularity of 
tufted cells underlies distinct afferent-evoked activity of olfactory bulb mitral and tufted cells. J Physiol 592:2097-2118. CrossRef Medline

Burton SD, Urban NN (2015) Rapid Feedforward Inhibition and Asynchronous Excitation Regulate Granule Cell Activity in the Mammalian Main Olfactory Bulb. J Neurosci 35:14103-14122. CrossRef Medline

Carlson GC, Shipley MT, Keller A (2000) Long-lasting depolarizations in mitral cells of the rat olfactory bulb. J Neurosci 20:2011-2021. Medline

Cheetham CE, Grier BD, Belluscio L (2015) Bulk regional viral injection in neonatal mice enables structural and functional interrogation of defined neuronal populations throughout targeted brain areas. Front Neural Circuits 9:72. CrossRef Medline

Cummings DM, Belluscio L (2010) Continuous neural plasticity in the olfactory intrabulbar circuitry. J Neurosci 30:9172-9180. CrossRef Medline

De Saint Jan D, Hirnet D, Westbrook GL, Charpak S (2009) External tufted cells drive the output of olfactory bulb glomeruli. J Neurosci 29:20432052. CrossRef Medline

Devore S, Linster C (2012) Noradrenergic and cholinergic modulation of olfactory bulb sensory processing. Front Behav Neurosci 6:52. CrossRef Medline

D'Souza RD, Vijayaraghavan S (2014) Paying attention to smell: cholinergic signaling in the olfactory bulb. Front Synaptic Neurosci 6:21. CrossRef Medline

Eyre MD, Antal M, Nusser Z (2008) Distinct deep short-axon cell subtypes of the main olfactory bulb provide novel intrabulbar and extrabulbar GABAergic connections. J Neurosci 28:8217-8229. CrossRef Medline

Eyre MD, Kerti K, Nusser Z (2009) Molecular diversity of deep short-axon cells of the rat main olfactory bulb. Eur J Neurosci 29:1397-1407. CrossRef Medline

Figl A, Cohen BN (2000) The subunit dominates the relaxation kinetics of heteromeric neuronal nicotinic receptors. J Physiol 524:685-699. CrossRef Medline

Fukunaga I, Berning M, Kollo M, Schmaltz A, Schaefer AT (2012) Two distinct channels of olfactory bulb output. Neuron 75:320-329. CrossRef Medline

Fukunaga I, Herb JT, Kollo M, Boyden ES, Schaefer AT (2014) Independent control of gamma and theta activity by distinct interneuron networks in the olfactory bulb. Nat Neurosci 17:1208-1216. CrossRef Medline

Geramita MA, Burton SD, Urban NN (2016) Distinct lateral inhibitory circuits drive parallel processing of sensory information in the mammalian olfactory bulb. eLife 5. pii: e16039. CrossRef Medline

Gire DH, Schoppa NE (2009) Control of on/off glomerular signaling by a local GABAergic microcircuit in the olfactory bulb. J Neurosci 29:1345413464. CrossRef Medline

Gong S, Doughty M, Harbaugh CR, Cummins A, Hatten ME, Heintz N, Gerfen CR (2007) Targeting Cre recombinase to specific neuron populations with bacterial artificial chromosome constructs. J Neurosci 27: 9817-9823. CrossRef Medline

Gschwend O, Abraham NM, Lagier S, Begnaud F, Rodriguez I, Carleton A (2015) Neuronal pattern separation in the olfactory bulb improves odor discrimination learning. Nat Neurosci 18:1474-1482. CrossRef Medline

Hayar A, Karnup S, Ennis M, Shipley MT (2004) External tufted cells: a major excitatory element that coordinates glomerular activity. J Neurosci 24:6676-6685. CrossRef Medline

$\mathrm{Hu}$ H, Gan J, Jonas P (2014) Interneurons. Fast-spiking, parvalbumin ${ }^{+}$ GABAergic interneurons: from cellular design to microcircuit function. Science 345:1255263. CrossRef Medline

Ishii K, Wong JK, Sumikawa K (2005) Comparison of alpha2 nicotinic acetylcholine receptor subunit mRNA expression in the central nervous system of rats and mice. J Comp Neurol 493:241-260. CrossRef Medline

Jia Y, Yamazaki Y, Nakauchi S, Sumikawa K (2009) $\alpha 2$ Nicotine receptors function as a molecular switch to continuously excite a subset of interneurons in rat hippocampal circuits. Eur J Neurosci 29:1588-1603. CrossRef Medline

Jiang X, Shen S, Cadwell CR, Berens P, Sinz F, Ecker AS, Patel S, Tolias AS (2015) Principles of connectivity among morphologically defined cell types in adult neocortex. Science 350:aac9462. CrossRef Medline

Kay LM, Lazzara P (2010) How global are olfactory bulb oscillations? J Neurophysiol 104:1768-1773. CrossRef Medline

Kubota Y (2014) Untangling GABAergic wiring in the cortical microcircuit. Curr Opin Neurobiol 26:7-14. CrossRef Medline

Leão RN, Mikulovic S, Leão KE, Munguba H, Gezelius H, Enjin A, Patra K, Eriksson A, Loew LM, Tort AB, Kullander K (2012) OLM interneurons differentially modulate CA3 and entorhinal inputs to hippocampal CA1 neurons. Nat Neurosci 15:1524-1530. CrossRef Medline

Letzkus JJ, Wolff SB, Lüthi A (2015) Disinhibition, a circuit mechanism for associative learning and memory. Neuron 88:264-276. CrossRef Medline

Linster C, Cleland TA (2016) Neuromodulation of olfactory transformations. Curr Opin Neurobiol 40:170-177. CrossRef Medline

Linster C, Fontanini A (2014) Functional neuromodulation of chemosensation in vertebrates. Curr Opin Neurobiol 29:82-87. CrossRef Medline

Liu S, Shao Z, Puche A, Wachowiak M, Rothermel M, Shipley MT (2015) Muscarinic receptors modulate dendrodendritic inhibitory synapses to sculpt glomerular output. J Neurosci 35:5680-5692. CrossRef Medline

Liu WL, Shipley MT (1994) Intrabulbar associational system in the rat olfactory bulb comprises cholecystokinin-containing tufted cells that synapse onto the dendrites of GABAergic granule cells. J Comp Neurol 346: 541-558. CrossRef Medline

Lodovichi C, Belluscio L, Katz LC (2003) Functional topography of connections linking mirror-symmetric maps in the mouse olfactory bulb. Neuron 38:265-276. CrossRef Medline

Lorincz A, Nusser Z (2008) Cell-type-dependent molecular composition of the axon initial segment. J Neurosci 28:14329-14340. CrossRef Medline

Lovett-Barron M, Kaifosh P, Kheirbek MA, Danielson N, Zaremba JD, Reardon TR, Turi GF, Hen R, Zemelman BV, Losonczy A (2014) Dendritic inhibition in the hippocampus supports fear learning. Science 343:857863. CrossRef Medline

Macrides F, Davis BJ, Youngs WM, Nadi NS, Margolis FL (1981) Cholinergic and catecholaminergic afferents to the olfactory bulb in the hamster: a neuroanatomical, biochemical, and histochemical investigation. J Comp Neurol 203:495-514. CrossRef Medline

Macrides F, Schoenfeld TA, Marchand JE, Clancy AN (1985) Evidence for morphologically, neurochemically and functionally heterogeneous classes of mitral and tufted cells in the olfactory bulb. Chemical Senses 10:175-202. CrossRef

Madisen L, Zwingman TA, Sunkin SM, Oh SW, Zariwala HA, Gu H, Ng LL, Palmiter RD, Hawrylycz MJ, Jones AR, Lein ES, Zeng H (2010) A robust and high-throughput Cre reporting and characterization system for the whole mouse brain. Nat Neurosci 13:133-140. CrossRef Medline

Mainland JD, Lundström JN, Reisert J, Lowe G (2014) From molecule to mind: an integrative perspective on odor intensity. Trends Neurosci 37: 443-454. CrossRef Medline

Markopoulos F, Rokni D, Gire DH, Murthy VN (2012) Functional properties of cortical feedback projections to the olfactory bulb. Neuron 76: 1175-1188. CrossRef Medline

Marks CA, Cheng K, Cummings DM, Belluscio L (2006) Activitydependent plasticity in the olfactory intrabulbar map. J Neurosci 26 : 11257-11266. CrossRef Medline

Miyamichi K, Shlomai-Fuchs Y, Shu M, Weissbourd BC, Luo L, Mizrahi A (2013) Dissecting local circuits: parvalbumin interneurons underlie broad feedback control of olfactory bulb output. Neuron 80:1232-1245. CrossRef Medline

Murayama M, Pérez-Garci E, Nevian T, Bock T, Senn W, Larkum ME (2009) Dendritic encoding of sensory stimuli controlled by deep cortical interneurons. Nature 457:1137-1141. CrossRef Medline

Nagayama S, Homma R, Imamura F (2014) Neuronal organization of olfactory bulb circuits. Front Neural Circuits 8:98. CrossRef Medline

Najac M, Sanz Diez A, Kumar A, Benito N, Charpak S, De Saint Jan D (2015) Intraglomerular lateral inhibition promotes spike timing variability in principal neurons of the olfactory bulb. J Neurosci 35:4319-4331. CrossRef Medline

Nunez-Parra A, Maurer RK, Krahe K, Smith RS, Araneda RC (2013) Disruption of centrifugal inhibition to olfactory bulb granule cells impairs olfactory discrimination. Proc Natl Acad Sci U S A 110:14777-14782. CrossRef Medline

Parrish-Aungst S, Shipley MT, Erdelyi F, Szabo G, Puche AC (2007) Quantitative analysis of neuronal diversity in the mouse olfactory bulb. J Comp Neurol 501:825-836. CrossRef Medline

Parsa PV, D'Souza RD, Vijayaraghavan S (2015) Signaling between periglomerular cells reveals a bimodal role for GABA in modulating glomerular microcircuitry in the olfactory bulb. Proc Natl Acad Sci U S A 112:94789483. CrossRef Medline

Pinto DJ, Brumberg JC, Simons DJ, Ermentrout GB (1996) A quantitative population model of whisker barrels: re-examining the Wilson-Cowan equations. J Comput Neurosci 3:247-264. CrossRef Medline 
Pouille F, Scanziani M (2001) Enforcement of temporal fidelity in pyramidal cells by somatic feed-forward inhibition. Science 293:1159-1163. CrossRef Medline

Pressler RT, Rozman PA, Strowbridge BW (2013) Voltage-dependent intrinsic bursting in olfactory bulb Golgi cells. Learn Mem 20:459-466. CrossRef Medline

Shao Z, Puche AC, Kiyokage E, Szabo G, Shipley MT (2009) Two GABAergic intraglomerular circuits differentially regulate tonic and phasic presynaptic inhibition of olfactory nerve terminals. J Neurophysiol 101: 1988-2001. CrossRef Medline

Shipley MT, Adamek GD (1984) The connections of the mouse olfactory bulb: a study using orthograde and retrograde transport of wheat germ agglutinin conjugated to horseradish peroxidase. Brain Res Bull 12:669688. CrossRef Medline

Smith RS, Hu R, DeSouza A, Eberly CL, Krahe K, Chan W, Araneda RC
(2015) Differential muscarinic modulation in the olfactory bulb. J Neurosci 35:10773-10785. CrossRef Medline

Smith TC, Jahr CE (2002) Self-inhibition of olfactory bulb neurons. Nat Neurosci 5:760-766. Medline

Tamás G, Lorincz A, Simon A, Szabadics J (2003) Identified sources and targets of slow inhibition in the neocortex. Science 299:1902-1905. CrossRef Medline

Wachowiak M (2011) All in a sniff: olfaction as a model for active sensing. Neuron 71:962-973. CrossRef Medline

Wester JC, McBain CJ (2014) Behavioral state-dependent modulation of distinct interneuron subtypes and consequences for circuit function. Curr Opin Neurobiol 29:118-125. CrossRef Medline

Zhou Z, Belluscio L (2012) Coding odorant concentration through activation timing between the medial and lateral olfactory bulb. Cell Rep 2:1143-1150. CrossRef Medline 